\title{
STABILITY OF SOLUTIONS TO GENERALIZED FORCHHEIMER EQUATIONS OF ANY DEGREE
}

\section{By}

\section{Luan Hoang, Akif Ibragimov, Thinh Kieu, Zeev Sobol}

\section{IMA Preprint Series \#2391}

(APRIL 2012)

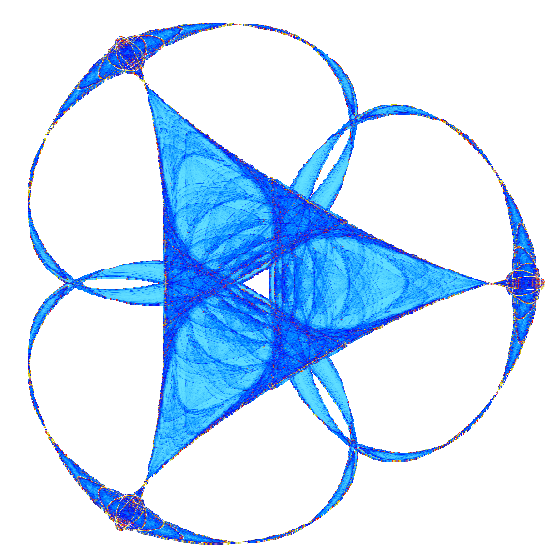

INSTITUTE FOR MATHEMATICS AND ITS APPLICATIONS

UNIVERSITY OF MINNESOTA 400 Lind Hall

207 Church Street S.E.

Minneapolis, Minnesota 55455-0436

Phone: 612-624-6066 Fax: 612-626-7370

URL: http://www.ima.umn.edu 


\title{
STABILITY OF SOLUTIONS TO GENERALIZED FORCHHEIMER EQUATIONS OF ANY DEGREE
}

\author{
LUAN HOANG $^{\dagger, *}$, AKIF IBRAGIMOV $^{\dagger}$, THINH KIEU $^{\dagger}$ AND ZEEV SOBOL ${ }^{\ddagger}$
}

\begin{abstract}
The non-linear Forchheimer equations are considered as laws of hydrodynamics in porous media in case of high Reynolds numbers, when the fluid flows deviate from the ubiquitous Darcy's law. In this article, the dynamics of generalized Forchheimer equations for slightly compressible fluids are studied by means of the resulting initial boundary value problem for the pressure. We prove that the solutions depend continuously on the boundary data and the Forchheimer polynomials both in finite time and at time infinity. In contrast to related long-time dynamics results which are in the $L^{2}$ context and require a restriction on the degree of the Forchheimer polynomial, the results obtained here are for general $L^{\alpha}$-spaces and without this degree restriction. New bounds for the solutions are established in $L^{\alpha}$-norm for all $\alpha \geq 1$, and then are used to improve estimates for their spatial and time derivatives. New Poincaré-Sobolev inequalities and non-linear Gronwall-type estimates for non-linear differential inequalities are utilized to achieve better asymptotic bounds.
\end{abstract}

\section{CONTENTS}

1. Introduction 1

2. Background and supplementaries 4

3. Existence results 11

4. Estimates of solutions 23

5. Dependence on the boundary data 35

6. Dependence on the Forchheimer polynomial 47

$\begin{array}{ll}\text { Appendix A. } & 60\end{array}$

References $\quad 62$

\section{INTRODUCTION}

Fluid flows in porous media are usually described by Darcy's law which is a linear relation between velocity $u$ and pressure gradient $\nabla p$. However this law does not hold in many cases, for instance, when the flows have high velocity or the media have fractures. Non-linear relations are used instead to model the fluid filtration in those situations.

Date: April 15, 2012.

2010 Mathematics Subject Classification. 35B30, 35B35, 35B40, 35K55, 35K65, 35Q35, 34C11.

Key words and phrases. Darcy-Forchheimer equation, porous media, asymptotic, stability, degenerate parabolic equation, Poincaré-Sobolev inequality, non-linear differential inequality. 
The common non-linear models are the so-called Forchheimer (or Darcy-Forchheimer) equations; specifically, $u$ and $\nabla p$ satisfy the equation $g(|u|) u=-\nabla p$ where $g(s)$ is a polynomial (c.f. [4,17]). Historically speaking, Darcy in his pioneering book [10] already used such a non-linear equation to match experimental data. While Darcy's law, even with its restriction, is well-established in literature as the basic equation for hydrodynamics in porous media, mathematical studies of the Forchheimer equations only gained attention in the 1990s (see e.g. $[9,19]$ ). Since then there have been a growing number of articles studying them and their variations - the Brinkman-Forchheimer equations - from applied and theoretical points of view. Interested reader can find references in [21]; among recent articles are [7, 8, 15, 18, 22]; for numerical studies, see e.g. [3]. Most of them are devoted to incompressible fluids. In contrast, this article continues our previous studies $[2,13,14]$ of slightly compressible fluid flows subject to Forchheimer equations.

Although the original and commonly used Forchheimer equations - the two term, three term and power laws - are only for a polynomial $g(s)$ of up to second degree, there is a need, from practical and theoretical point of view, to allow $g$ to be a generalized polynomial with positive coefficients of higher degrees. In this case corresponding equation is called generalized Forchheimer equation (see [2]) and $g(s)$ is called the Forchheimer polynomial. The generalized Forchheimer equation can be inverted to $u=-K(|\nabla p|) \nabla p$ with conductivity function $K$ degenerating for large $\nabla p$. Namely, $K(\xi) \sim\left(1+\xi^{a}\right)^{-1}$, where $a=\frac{\operatorname{deg}(g)}{(1+\operatorname{deg}(g))}$ with $\operatorname{deg}(g)$ denoting the degree of the polynomial $g$. For slightly compressible fluids, the description of fluid dynamics can be deduced, with a slight simplification, to a degenerate parabolic equation for the pressure $p(x, t)$ :

$$
\frac{\partial p}{\partial t}=\nabla \cdot(K(|\nabla p|) \nabla p) \text { in } U \times(0, \infty)
$$

Degenerate equations of this type are extensively studied mostly for their solutions' spatially local behavior and/or finite time properties (see [11] and references therein, and also, for e.g., [1] for a localization result).

Here we study the initial boundary value problem (IBVP) for (1.1) with Dirichlet boundary data on an open bounded domain $U$ in $\mathbb{R}^{n}$ and focus on its long-time dynamics. In our previous works [2,13], see also [14], we establish the continuous dependence of the solutions of (1.1) in $L^{2}$ - and $W^{1,2-a}$-norms on the initial, boundary data and on the Forchheimer polynomials. To effectively study the asymptotic dynamics of the solutions, a technical Degree Condition (DC) is imposed, namely, $\operatorname{deg}(g) \leq \frac{n}{n-2}$. This condition also arises naturally in studies of degenerate parabolic equations. (See e.g. $[11,12]$ for the cruciality of such condition in establishing Harnack inequalities.)

Our goals in this article are to establish a $L^{\alpha}$-theory for $\alpha \neq 2$, as a counterpart for the $L^{2}$-results, and to explore the problem when the DC is not met - the case we now refer 
to as NDC. Two observations that support our effort: (a) The DC is not needed for timeinvariant velocity field (see [2]); and (b) basic numerical simulations hint at the stability at time infinity of the solution in $L^{\alpha}$-norm.

In this paper, we establish the continuous dependence of the solution of (1.1) on either the boundary data or coefficients of the Forchheimer polynomial in the space $L^{\alpha}(U)$ for $\alpha \geq 1$ and in $W^{1,2-a}(U)$. We will mainly focus on the NDC case. In the DC case we obtain sharper estimates which significantly generalize previous $L^{2}$-estimates in [13].

The main features of our results are:

(i) New $L^{\alpha}$-estimates of the solutions for all $\alpha \geq 1$. Because of the order of degeneracy, the differential inequality for the $L^{\alpha}$-norm has a weak dissipative term, see (4.11). We use a new Poincaré-Sobolev inequality for the mixed term (of derivatives) $|\nabla \bar{p}|^{2-a}|\bar{p}|^{\alpha-2}$ to take advantage of this weak dissipation to gain much sharper estimates. Here $\bar{p}$ is the solution $p$ adjusted by the Dirichlet data.

(ii) The continuous dependence of the solution on the time-dependent boundary data in $L^{\alpha}$-norm. Another new weighted Poincaré-Sobolev inequality is used, c.f. Lemma 2.5. Particularly, in the NDC case (part (ii) of the mentioned lemma) the inequality is highly non-linear and involves, in addition to the weight $K(\xi(x))$, a compensating term $|u|^{\theta_{2} \alpha}$. We then utilize a new non-linear Gronwall-type estimates, c.f. Lemma A.1, to explore the resulting differential inequality.

(iii) The continuous dependence of the solution on the Forchheimer polynomial in $L^{\alpha}$ norm. This requires estimates of the cross-terms $\left|\nabla p_{1}\right|^{2-a}\left|\bar{p}_{2}\right|^{\alpha-2}$ and $\left|\nabla p_{2}\right|^{2-a}\left|\bar{p}_{1}\right|^{\alpha-2}$ of individual solutions $p_{1}$ and $p_{2}$. By using the structure of the equation we bound them by the mixed terms which are dealt with in (i).

(iv) The continuous dependence of the pressure gradient in $L^{2-a}$-norm with respect to the boundary data and the Forchheimer polynomials.

(v) The above continuous dependence results are established in both DC and NDC cases.

The paper is organized as follows. In section 2 we recall the main definitions and relevant results from $[2,13,14]$. We then obtain some inequalities of Poincaré-Sobolev type in Lemmas 2.3 and 2.5. They are suitable for the non-linear degenerate parabolic equation (1.1), and are essential to our analysis of the solutions, particularly their asymptotic behavior. In section 3 , using the theory of monotone operators $[5,16,20,23]$, we prove the global existence of weak solutions in $C\left([0, \infty), L^{\alpha}(U)\right)$ and $L_{l o c}^{2-a}\left([0, \infty), W^{1,2-a}(U)\right)$. In section 4 we derive more refined estimates of the solutions to $(1.1)$ in $L^{\alpha}(U)$ for all $\alpha \geq 1$ with explicit dependence on the time-dependent boundary data. (For this, the initial data are required to belong to $L^{\widehat{\alpha}}(U)$ with $\widehat{\alpha} \geq \alpha$, see Definition 4.2.) These are used to improve estimates of the pressure's spatial and time derivatives, especially for the NDC case. Moreover, asymptotic bounds for the solutions are obtained. As a consequence, we 
are able to estimate the mixed terms $\int_{U}|\nabla p|^{2-a}|p|^{\alpha} d x$ for arbitrarily large $\alpha$. This result will be key to proving the structural stability in section 6. In section 5 we establish the continuous dependence of solutions on the boundary data in $L^{\alpha}$ - and $W^{1,2-a}$ - norms. To investigate this for large time we use a generalization of Gronwall's inequality for certain non-linear differential inequalities at finite time as well as at time infinity. The upper bounds of difference between two solutions for large time and their limits superior when $t \rightarrow \infty$ are given in Theorems 5.2-5.6. It is noteworthy that these bounds are independent of the initial data. In section 6 we prove various theorems on structural stability for equation (1.1). More precisely, we show that the solution depends continuously on the coefficients of the Forchheimer polynomial both for finite time intervals and for time infinity and in both DC and NDC cases. Unlike previous $L^{2}$-estimates in [13] one must control the cross-terms $\int_{U}\left|\nabla p_{1}\right|^{2-a}\left|\bar{p}_{2}\right|^{\alpha-2} d x$ and $\int_{U}\left|\nabla p_{2}\right|^{2-a}\left|\bar{p}_{1}\right|^{\alpha-2} d x$, see (6.11) of Lemma 6.1. This cannot be achieved directly from previous sections' estimates. However by exploring the structure of equation (1.1) we can estimate these integrals using the mixed terms $\int_{U}\left|\nabla p_{1}\right|^{2-a}\left|\bar{p}_{1}\right|^{\gamma} d x$ and $\int_{U}\left|\nabla p_{2}\right|^{2-a}\left|\bar{p}_{2}\right|^{\gamma} d x$ for possibly very large $\gamma$; the latter integrals are already estimated in section 4. The Appendix generalizes our previous results in $[13,14]$ to give bounds for solutions to a wider class of non-linear differential inequalities.

\section{BACKGROUND AND SUPPLEMENTARIES}

Consider a fluid in a porous medium occupying a bounded domain $U$ in space $\mathbb{R}^{n}$. Throughout this paper, $n \geq 2$ even though for physics problems $n=2$ or 3 . Let $x \in \mathbb{R}^{n}$ and $t \in \mathbb{R}$ be the spatial and time variables. The fluid flow has velocity $u(x, t) \in \mathbb{R}^{n}$, pressure $p(x, t) \in \mathbb{R}$ and density $\rho(x, t) \in \mathbb{R}^{+}=[0, \infty)$.

A generalized Forchheimer equation is

$$
g(|u|) u=-\nabla p
$$

where $g(s) \geq 0$ is a function defined on $[0, \infty)$. It is considered as a momentum equation and is studied in $[2,13,14]$. When $g(s)=\alpha, \alpha+\beta s, \alpha+\beta s+\gamma s^{2}, \alpha+\gamma_{m} s^{m-1}$, where $\alpha, \beta, \gamma, m, \gamma_{m}$ are empirical constants, we have Darcy's law, Forchheimer's two term, three term and power laws, respectively. In this paper, we study the case when the function $g$ in (2.1) is a generalized polynomial with non-negative coefficients. More precisely, the function $g: \mathbb{R}^{+} \rightarrow \mathbb{R}^{+}$is of the form

$$
g(s)=a_{0} s^{\alpha_{0}}+a_{1} s^{\alpha_{1}}+\cdots+a_{N} s^{\alpha_{N}}, \quad s \geq 0,
$$

where $N \geq 1, \alpha_{0}=0<\alpha_{1}<\cdots<\alpha_{N}$ are fixed real numbers, the coefficients $a_{0}, \ldots, a_{N}$ are non-negative numbers with $a_{0}>0$ and $a_{N}>0$. From (2.1) one can solve for $u$ in terms of $\nabla p$ and obtain a non-linear version of Darcy's equation:

$$
u=-K(|\nabla p|) \nabla p,
$$


where the function $K: \mathbb{R}^{+} \rightarrow \mathbb{R}^{+}$is defined for $\xi \geq 0$ by

$K(\xi)=\frac{1}{g(s(\xi))}$, with $s=s(\xi)$ being the unique non-negative solution of $s g(s)=\xi$.

The number $\alpha_{N}$ is the degree of $g$ and is denoted by $\operatorname{deg}(g)$. The vector of powers in (2.2) is denoted by $\vec{\alpha}=\left(\alpha_{0}, \ldots, \alpha_{N}\right)$ and the vector $\vec{a}=\left(a_{0}, \ldots, a_{N}\right)$ is referred to as the coefficient vector. When the dependence on $\vec{a}$ needs to be specified, we use notation $g(s, \vec{a}), K(\xi, \vec{a})$ to denote the corresponding functions in (2.2) and (2.4).

In addition to (2.1) we have the equation of continuity

$$
\frac{\partial \rho}{\partial t}+\nabla \cdot(\rho u)=0
$$

and the equation of state which, for slightly compressible fluids, is

$$
\frac{d \rho}{d p}=\frac{\rho}{\kappa}, \quad \kappa>0
$$

Substituting (2.3) and (2.5) into (2.6) we obtain a scalar equation for the pressure:

$$
\frac{\partial p}{\partial t}=\kappa \nabla \cdot(K(|\nabla p|) \nabla p)+K(|\nabla p|)|\nabla p|^{2} .
$$

One the right hand side of (2.7) the constant $\kappa$ is very large for most slightly compressible fluid in porous media, hence we neglect its second term and study the following reduced equation

$$
\frac{\partial p}{\partial t}=\kappa \nabla \cdot(K(|\nabla p|) \nabla p)
$$

(This simplification is used commonly in petroleum engineering.) By scaling the time variable $t \rightarrow \kappa t$, we can assume throughout that $\kappa=1$.

The class of functions $g(s)$ as in (2.2) is denoted by $F P(N, \vec{\alpha})$ which is the abbreviation of "Forchheimer polynomials". When the function $g$ in $(2.1)$ is one of the $g(s)$ in (2.2), it is referred to as the Forchheimer polynomial.

Let $g=g(s, \vec{a})$ in $F P(N, \vec{\alpha})$. The following exponent is frequently used in our calculations

$$
a=\frac{\alpha_{N}}{\alpha_{N}+1} \in(0,1) .
$$

The function $K(\xi)$ in (2.4) has the following properties: it is decreasing in $\xi$, maps $[0, \infty)$ onto $\left(0, \frac{1}{a_{0}}\right]$ and

$$
\begin{aligned}
\frac{d_{1}}{(1+\xi)^{a}} & \leq K(\xi) \leq \frac{d_{2}}{(1+\xi)^{a}}, \\
d_{3}\left(\xi^{2-a}-1\right) & \leq K(\xi) \xi^{2} \leq d_{2} \xi^{2-a},
\end{aligned}
$$

where $d_{1}, d_{2}, d_{3}$ are positive constants depending on $\vec{\alpha}$ and $\vec{a}$.

As in $[2,13,14]$, we define

$$
H(\xi)=\int_{0}^{\xi^{2}} K(\sqrt{s}) d s \quad \text { for } \xi \geq 0
$$


The function $H(\xi)$ can be compared with $\xi$ and $K(\xi)$ by

$$
K(\xi) \xi^{2} \leq H(\xi) \leq 2 K(\xi) \xi^{2}
$$

and hence, as a consequence of (2.11) and (2.12), we have

$$
d_{3}\left(\xi^{2-a}-1\right) \leq H(\xi) \leq 2 d_{2} \xi^{2-a} .
$$

Since $H^{\prime}(\xi)=2 \xi K(\xi)$ is non-negative and, by Lemma III.5 of [2], increasing,

$$
H(\xi) \text { is an increasing and convex function on }[0, \infty) .
$$

We recall here Lemma 2.3 in [13] on an important monotonicity property.

Lemma 2.1 (c.f. [13]). (i) For any $y, y^{\prime} \in \mathbb{R}^{n}$,

$$
\left(K\left(\left|y^{\prime}\right|\right) y^{\prime}-K(|y|) y\right) \cdot\left(y^{\prime}-y\right) \geq(1-a) K\left(\max \left\{|y|,\left|y^{\prime}\right|\right\}\right)\left|y^{\prime}-y\right|^{2} .
$$

(ii) For two functions $p_{1}$ and $p_{2}$ defined on $U$, one has

$$
\begin{aligned}
& \int_{U}\left(K\left(\left|\nabla p_{1}\right|\right) \nabla p_{1}-K\left(\left|\nabla p_{2}\right|\right) \nabla p_{2}\right) \cdot\left(\nabla p_{1}-\nabla p_{2}\right) d x \\
& \geq(1-a) \int_{U} K\left(\max \left\{\left|\nabla p_{1}\right|,\left|\nabla p_{2}\right|\right\}\right)\left|\nabla p_{1}-\nabla p_{2}\right|^{2} d x \\
& \geq d_{4}\left[\int_{U}\left|\nabla p_{1}-\nabla p_{2}\right|^{2-a} d x\right]^{\frac{2}{2-a}}\left(1+\max \left\{\left\|\nabla p_{1}\right\|_{L^{2-a}},\left\|\nabla p_{2}\right\|_{L^{2-a}}\right\}\right)^{-a},
\end{aligned}
$$

where $d_{4}=d_{4}(\vec{\alpha}, \vec{a}, n, U)>0$.

Definition 2.2. We will refer to the following inequality as the Degree Condition

$$
\operatorname{deg}(g) \leq \frac{4}{n-2}
$$

We label the negation of (DC) by (NDC), that is,

$$
\operatorname{deg}(g)>\frac{4}{n-2}
$$

We will use the phrase "the DC case", respectively "the NDC case", to refer to the case when condition (DC), respectively condition (NDC), holds.

We establish below a couple of Poincaré-Sobolev inequalities which are suitable to our type of degeneracy in (2.8) and are essential to our estimates in subsequent sections. We first recall the classical Poincaré-Sobolev inequality.

Let $\stackrel{\circ}{W}^{1, r}(U)$ be the space of functions in $W^{1, r}(U)$ with vanishing traces on the boundary. If $1 \leq r<n$, then by Sobolev's imbedding theorem and Poincaré's inequality, we have

$$
\|f\|_{L^{r^{*}(U)}} \leq C\|\nabla f\|_{L^{r}(U)} \text { for all } f \in \stackrel{\circ}{W}^{1, r}(U),
$$

where the constant $C$ depends on $r, n$ and the domain $U$, and

$$
r^{*}=n r /(n-r) \text {. }
$$

Throughout, $r_{0}$ is the conjugate of $(2-a)^{*}$, that is, $\frac{1}{r_{0}}+\frac{1}{(2-a)^{*}}=1$. 
We denote

$$
\alpha_{*}=\frac{n a}{2-a}=\frac{n \alpha_{N}}{\alpha_{N}+2}
$$

a threshold exponent for the validity of our inequalities below.

One can easily verify that

$$
\begin{aligned}
(D C) & \Leftrightarrow 2 \leq(2-a)^{*} \Leftrightarrow r_{0} \leq 2 \Leftrightarrow \alpha_{*} \leq 2, \\
(N D C) & \Leftrightarrow 2>(2-a)^{*} \Leftrightarrow r_{0}>2 \Leftrightarrow \alpha_{*}>2 .
\end{aligned}
$$

In the following, $C$ denotes a generic positive constant whose value may vary from one line to another.

Lemma 2.3. Let $U$ be an open, bounded domain in $\mathbb{R}^{n}$ and $\alpha \geq \max \left\{2, \alpha_{*}\right\}$. There exists $c_{1}=c_{1}(\alpha, \vec{\alpha}, \vec{a}, n, U)>0$ such that if $u$ is a function on $U$ vanishing on the boundary $\partial U$, then

$$
\int_{U}|u|^{\alpha} d x \leq c_{1}\left\{\int_{U} K(|\nabla u|)|\nabla u|^{2}|u|^{\alpha-2} d x+\left(\int_{U} K(|\nabla u|)|\nabla u|^{2}|u|^{\alpha-2} d x\right)^{\gamma_{0}}\right\},
$$

where $\gamma_{0}=\gamma_{0}(\alpha)=\frac{\alpha}{\alpha-a}$; consequently,

$$
\int_{U}|\nabla u|^{2-a}|u|^{\alpha-2} d x \geq c_{2}\left(\left(\int_{U}|u|^{\alpha} d x\right)^{\frac{1}{\gamma_{0}}}-1\right)
$$

where $c_{2}=c_{2}(\alpha, \vec{\alpha}, \vec{a}, n, U)>0$.

Proof. Let $\xi=\xi(x)$ be a non-negative function on $U$. Let $\delta=1-\frac{a}{2} \in(0,1), r=2-a \in$ $(1,2)$ and $m=\frac{\alpha-a}{2-a} \geq 1$. Applying Poincaré-Sobolev's inequality (2.16) to the function $|u|^{m}$, we have

$$
\left(\int_{U}|u|^{m r^{*}} d x\right)^{\frac{1}{r^{*}}} \leq C\left[\int_{U}\left(\nabla|u|^{m}\right)^{r} d x\right]^{\frac{1}{r}} \leq C\left[\int_{U}|\nabla u|^{r} K^{\delta}(\xi) \cdot K^{-\delta}(\xi)|u|^{(m-1) r} d x\right]^{\frac{1}{r}} .
$$

Applying Hölder's inequality with powers $1 / \delta$ and $1 /(1-\delta)$ gives

$$
\left(\int_{U}|u|^{m r^{*}} d x\right)^{\frac{1}{r^{*}}} \leq C\left[\int_{U}|\nabla u|^{\frac{r}{\delta}} K(\xi)|u|^{(m-1) r} d x\right]^{\frac{\delta}{r}}\left[\int_{U} K^{\frac{-\delta}{1-\delta}}(\xi)|u|^{(m-1) r} d x\right]^{\frac{1-\delta}{r}} .
$$

Using (2.10) to estimate $K^{\frac{-\delta}{1-\delta}}(\xi)$ yields

$$
\begin{aligned}
\left(\int_{U}|u|^{m r^{*}} d x\right)^{\frac{1}{r^{*}}} & \leq C\left[\int_{U}|\nabla u|^{\frac{r}{\delta}} K(\xi)|u|^{(m-1) r} d x\right]^{\frac{\delta}{r}}\left[\int_{U}(1+\xi)^{\frac{a \delta}{1-\delta}}|u|^{(m-1) r} d x\right]^{\frac{1-\delta}{r}} \\
& =C\left[\int_{U}|\nabla u|^{2} K(\xi)|u|^{\alpha-2} d x\right]^{\frac{1}{2}}\left[\int_{U}(1+\xi)^{2-a}|u|^{\alpha-2} d x\right]^{\frac{a}{2(2-a)}} .
\end{aligned}
$$

Using relation (2.11) to compare $(1+\xi)^{2-a}$ with $1+K(\xi) \xi^{2}$, we assert

$$
\left(\int_{U}|u|^{m r^{*}} d x\right)^{\frac{1}{r^{*}}} \leq C\left[\int_{U} K(\xi)|\nabla u|^{2}|u|^{\alpha-2} d x\right]^{\frac{1}{2}}\left[\int_{U}\left(1+K(\xi) \xi^{2}\right)|u|^{\alpha-2} d x\right]^{\frac{a}{2(2-a)}} \text {. }
$$


Since $\alpha \geq \alpha_{*}$, elementary calculations show $\alpha \leq m r^{*}$. Then using Holder's inequality and applying (2.20) with $\xi=|\nabla u|$ yield

$$
\begin{aligned}
& \int_{U}|u|^{\alpha} d x \leq C\left(\int_{U}|u|^{m r^{*}} d x\right)^{\frac{\alpha}{m r^{*}}} \\
& \leq C\left[\int_{U} K(|\nabla u|)|\nabla u|^{2}|u|^{\alpha-2} d x\right]^{\frac{\alpha(2-a)}{2(\alpha-a)}}\left[\int_{U}\left(1+K(|\nabla u|)|\nabla u|^{2}\right)|u|^{\alpha-2} d x\right]^{\frac{a \alpha}{2(\alpha-a)}} \\
& \leq C\left[\int_{U} K(|\nabla u|)|\nabla u|^{2}|u|^{\alpha-2} d x\right]^{\frac{\alpha(2-a)}{2(\alpha-a)}}\left[\int_{U}|u|^{\alpha-2} d x+\int_{U} K(|\nabla u|)|\nabla u|^{2}|u|^{\alpha-2} d x\right]^{\frac{a \alpha}{2(\alpha-a)}} .
\end{aligned}
$$

Hence

$$
\begin{aligned}
\int_{U}|u|^{\alpha} d x \leq & C\left[\int_{U} K(|\nabla u|)|\nabla u|^{2}|u|^{\alpha-2} d x\right]^{\frac{\alpha(2-a)}{2(\alpha-a)}}\left[\int_{U}|u|^{\alpha} d x\right]^{\frac{a(\alpha-2)}{2(\alpha-a)}} \\
& +C\left[\int_{U} K(|\nabla u|)|\nabla u|^{2}|u|^{\alpha-2} d x\right]^{\frac{\alpha}{\alpha-a}} .
\end{aligned}
$$

Applying Young's inequality with powers $\frac{2(\alpha-a)}{\alpha(2-a)}$ and $\frac{2(\alpha-a)}{a(2-a)}$ to the product term on the right-hand side of the previous inequality, we obtain

$$
\begin{aligned}
\int_{U}|u|^{\alpha} d x \leq & C \int_{U} K(|\nabla u|)|\nabla u|^{2}|u|^{\alpha-2} d x+\frac{1}{2} \int_{U}|u|^{\alpha} d x \\
& +C\left[\int_{U} K(|\nabla u|)|\nabla u|^{2}|u|^{\alpha-2} d x\right]^{\frac{\alpha}{\alpha-a}}
\end{aligned}
$$

and (2.18) follows as a consequence.

We define a family of non-linear functions $\varphi_{c, \gamma}$ by

$$
\varphi_{c, \gamma}(z)=c\left(z+z^{\gamma}\right), \quad \text { for } c>0, \gamma>0, z \geq 0 .
$$

Since $K(|\nabla u|)|\nabla u|^{2} \leq d_{2}|\nabla u|^{2-a}$ we have from (2.18) that

$$
\int_{U}|u|^{\alpha} d x \leq \varphi_{c, \gamma_{0}}\left(\int_{U}|\nabla u|^{2-a}|u|^{\alpha-2} d x\right),
$$

where $c=c_{1} \max \left\{d_{2}, d_{2}^{\gamma_{0}}\right\}$, and hence

$$
\int_{U}|\nabla u|^{2-a}|u|^{\alpha-2} d x \geq \varphi_{c, \gamma_{0}}^{-1}\left(\int_{U}|u|^{\alpha} d x\right) .
$$

For $z \geq 0$, let $y=\varphi_{c, \gamma_{0}}(z)=c\left(z+z^{\gamma_{0}}\right)$. Then $y \leq 1+C_{1} z^{\gamma_{0}}$, where $C_{1}=c+c^{\gamma_{0}}$, hence $z^{\gamma_{0}} \geq C_{1}^{-1}(y-1)$. Noticing that $1 / \gamma_{0}<1$, we assert $z \geq C_{1}^{-1 / \gamma_{0}}\left(y^{\frac{1}{\gamma_{0}}}-1\right)$, that is, $\varphi_{c, \gamma_{0}}^{-1}(y) \geq C_{1}^{-1 / \gamma_{0}}\left(y^{\frac{1}{\gamma_{0}}}-1\right)$. Therefore we obtain (2.19) from (2.22).

Remark 2.4. For $\alpha \geq \max \left\{2, \alpha_{*}\right\}$, a straightforward application of the imbedding from the Sobolev space $\stackrel{\circ}{W}^{1,2-a}(U)$ into $L^{\frac{\alpha(2-a)}{\alpha-a}}(U)$ to function $|u|^{\alpha}$ gives

$$
\int_{U}|u|^{\alpha} d x \leq C\left[\int_{U}|\nabla u|^{2-a}|u|^{\alpha-2} d x\right]^{\frac{\alpha}{2-a}},
$$




$$
\int_{U}|\nabla u|^{2-a}|u|^{\alpha-2} d x \geq C\left[\int_{U}|u|^{\alpha} d x\right]^{\frac{2-a}{\alpha}} .
$$

Since $1<\gamma_{0}=\frac{\alpha}{\alpha-a}<\frac{\alpha}{2-a}$, then roughly speaking, (2.18) is better than (2.23), and (2.19) is better than (2.24).

We now prove another Poincaré-Sobolev inequality with a specific weight.

Lemma 2.5. Let $U$ be an open bounded domain in $\mathbb{R}^{n}$ and $\xi=\xi(x)$ be a non-negative function defined on $U$. Assume $\alpha \geq 2$.

(i) In the DC case, there is a constant $c_{3}=c_{3}(\alpha, \vec{\alpha}, \vec{a}, n, U)>0$ such that

$$
\int_{U}|u|^{\alpha} d x \leq c_{3}\left[\int_{U} K(\xi)|\nabla u|^{2}|u|^{\alpha-2} d x\right]\left[1+\int_{U} \xi^{2-a} d x\right]^{\frac{a}{2-a}}
$$

for any function $u(x)$ vanishing on the boundary $\partial U$.

(ii) In the NDC case, given two numbers $\theta$ and $\theta_{1}$ that satisfy

$$
\theta>\frac{2}{(2-a)^{*}} \text { and } \max \left\{1, \frac{2 n}{n \theta+2}\right\} \leq \theta_{1}<2-a,
$$

there is a constant $c_{4}=c_{4}\left(\alpha, \theta, \theta_{1}, \vec{\alpha}, \vec{a}, n, U\right)>0$ such that

$$
\int_{U}|u|^{\alpha} d x \leq c_{4}\left[\int_{U} K(\xi)|\nabla u|^{2}|u|^{\alpha-2} d x\right]^{\frac{1}{\theta}}\left[1+\int_{U} \xi^{2-a}+|u|^{\theta_{2} \alpha} d x\right]^{\frac{2-\theta_{1}}{\theta \theta_{1}}}
$$

for any function $u(x)$ vanishing on the boundary $\partial U$, where

$$
\theta_{2}=\frac{\theta_{1}(\theta-1)(2-a)}{2\left(2-a-\theta_{1}\right)}>0
$$

Proof. Suppose $\theta_{1}$ and $m$ are two numbers that satisfy $1 \leq \theta_{1}<n$ and $\frac{\alpha}{m} \leq \theta_{1}^{*}$. By the standard Poincaré-Sobolev's inequality corresponding to the imbedding of $\mathscr{W}^{1, \theta_{1}}(U)$ into $L^{\frac{\alpha}{m}}(U)$, we have

$$
\int_{U}|u|^{\alpha} d x=\int_{U}\left(|u|^{m}\right)^{\frac{\alpha}{m}} d x \leq C\left[\left.\left.\int_{U}|\nabla| u\right|^{m}\right|^{\theta_{1}} d x\right]^{\frac{\alpha}{m \theta_{1}}} \leq C\left[\int_{U}|\nabla u|^{\theta_{1}}|u|^{(m-1) \theta_{1}} d x\right]^{\frac{\alpha}{m \theta_{1}}} .
$$

If $\delta_{1} \in[0,1]$ and $0<\theta_{1}<2$ then rewriting the above inequality as

$$
\int_{U}|u|^{\alpha} d x \leq C\left[\int_{U}\left(|\nabla u|^{\theta_{1}} K(\xi)^{\frac{\theta_{1}}{2}}|u|^{\delta_{1}(m-1) \theta_{1}}\right)\left(K(\xi)^{-\frac{\theta_{1}}{2}}|u|^{\left(1-\delta_{1}\right)(m-1) \theta_{1}}\right) d x\right]^{\frac{\alpha}{m \theta_{1}}}
$$

and then applying Holder's inequality with powers $2 / \theta_{1}$ and $2 /\left(2-\theta_{1}\right)$ give

$$
\int_{U}|u|^{\alpha} d x \leq C\left[\int_{U} K(\xi)|\nabla u|^{2}|u|^{2 \delta_{1}(m-1)} d x\right]^{\frac{\alpha}{2 m}}\left[\int_{U} K(\xi)^{-\frac{\theta_{1}}{2-\theta_{1}}}|u|^{\frac{2 \theta_{1}\left(1-\delta_{1}\right)(m-1)}{2-\theta_{1}}} d x\right]^{\frac{\alpha\left(2-\theta_{1}\right)}{2 \theta_{1} m}} .
$$


(i) Consider the DC case. Set $\theta_{1}=2-a, m=\frac{\alpha}{2}$ and $\delta_{1}=1$, then $1<\theta_{1}<2$ and $\alpha / m=2 \leq \theta_{1}^{*}$ thanks to (2.17). By (2.29) and (2.10) we have

$$
\begin{aligned}
\int_{U}|u|^{\alpha} d x & \leq C\left[\int_{U} K(\xi)|\nabla u|^{2}|u|^{\alpha-2} d x\right]\left[\int_{U} K(\xi)^{-\frac{2-a}{a}} d x\right]^{\frac{a}{2-a}} \\
& \leq C\left[\int_{U} K(\xi)|\nabla u|^{2}|u|^{\alpha-2} d x\right]\left[\int_{U}\left(1+\xi^{2-a}\right) d x\right]^{\frac{a}{2-a}},
\end{aligned}
$$

thus obtaining (2.25).

(ii) Next, we consider the NDC case. Note that $\theta>\frac{2}{(2-a)^{*}}$ is equivalent to $\frac{2 n}{n \theta+2}<2-a$, hence there always exists $\theta_{1}$ that satisfies the second condition in (2.26). Also, $\theta>1$ thanks to (2.17).

Set $m=\theta \alpha / 2$ and $\delta_{1}=(\alpha-2) /(2(m-1))$. Then $m>\alpha / 2 \geq 1$ and hence $\delta_{1} \in[0,1)$. Since $\theta_{1} \geq \frac{2 n}{n \theta+2}$, we have $2 m / \alpha=\theta \geq 2 / \theta_{1}^{*}$ or $\alpha / m \leq \theta_{1}^{*}$. Note that $2 \delta_{1}(m-1)=\alpha-2$ and $2\left(1-\delta_{1}\right)(m-1)=2(m-1)-(\alpha-2)=2 m-\alpha$. Therefore it follows from (2.29) that

$$
\begin{aligned}
\int_{U}|u|^{\alpha} d x & \leq\left[\int_{U} K(\xi)|\nabla u|^{2}|u|^{\alpha-2} d x\right]^{\frac{\alpha}{2 m}}\left[\int_{U} K(\xi)^{-\frac{\theta_{1}}{2-\theta_{1}}}|u|^{\frac{\theta_{1}(2 m-\alpha)}{2-\theta_{1}}} d x\right]^{\frac{\alpha\left(2-\theta_{1}\right)}{2 \theta_{1} m}} \\
& \leq\left[\int_{U} K(\xi)|\nabla u|^{2}|u|^{\alpha-2} d x\right]^{\frac{\alpha}{2 m}}\left[\int_{U}(1+\xi)^{\frac{a \theta_{1}}{2-\theta_{1}}}|u|^{\frac{\theta_{1}(2 m-\alpha)}{2-\theta_{1}}} d x\right]^{\frac{\alpha\left(2-\theta_{1}\right)}{2 \theta_{1} m}} .
\end{aligned}
$$

Since $\theta_{1}<2-a$ implies $\frac{a \theta_{1}}{2-\theta_{1}}<2-a$, we apply Young's inequality to obtain

$$
\int_{U}(1+\xi)^{\frac{a \theta_{1}}{2-\theta_{1}}}|u|^{\frac{\theta_{1}(2 m-\alpha)}{2-\theta_{1}}} d x \leq C \int_{U}(1+\xi)^{2-a}+|u|^{\frac{\theta_{1}(2 m-\alpha)(2-a)}{2\left(2-\theta_{1}-a\right)}} d x .
$$

Note that

$$
\frac{\theta_{1}(2 m-\alpha)(2-a)}{2\left(2-\theta_{1}-a\right)}=\theta_{2} \alpha
$$

Therefore we have from (2.30), (2.31) and (2.32) that

$$
\int_{U}|u|^{\alpha} d x \leq C\left[\int_{U} K(\xi)|\nabla u|^{2}|u|^{\alpha-2} d x\right]^{\frac{\alpha}{2 m}}\left[\int_{U}\left(1+|u|^{\theta_{2} \alpha}+\xi^{2-a}\right) d x\right]^{\frac{\alpha\left(2-\theta_{1}\right)}{2 m \theta_{1}}}
$$

which proves (2.27).

We derive below some simple but useful estimates for solutions of certain non-linear ordinary differential inequalities.

Definition 2.6. Given $f(t)$ defined on an interval $I \subset \mathbb{R}$. A function $F(t)$ is called an (upper) envelop of $f(t)$ on $I$ if $F(t) \geq f(t)$ for all $t \in I$. We denote by $E n v(f)$ a continuous, increasing envelop function of $f(t)$.

Lemma 2.7. Let $\theta>0$ and let $y(t) \geq 0, h(t)>0, f(t) \geq 0$ be continuous functions on $[0, \infty)$ that satisfy

$$
y^{\prime}(t) \leq-h(t) y(t)^{\theta}+f(t) \quad \text { for all } t>0 .
$$

Then

$$
y(t) \leq y(0)+[\operatorname{Env}(f(t) / h(t))]^{\frac{1}{\theta}} \text { for all } t \geq 0
$$


If $\int_{0}^{\infty} h(t) d t=\infty$ then

$$
\limsup _{t \rightarrow \infty} y(t) \leq \limsup _{t \rightarrow \infty}[f(t) / h(t)]^{\frac{1}{\theta}} .
$$

In the simple case when $y(t)=\int_{0}^{t} e^{-k(t-\tau)} f(\tau) d \tau$, where $k$ is a positive number, we have from (2.34) that

$$
\limsup _{t \rightarrow \infty} \int_{0}^{t} e^{-k(t-\tau)} f(\tau) d \tau \leq k^{-1} \limsup _{t \rightarrow \infty} f(t) .
$$

Lemma 2.7 is a special case of Lemma A.1 when applied to the function $\phi(s)=s^{1 / \theta}$. The reader is referred to Appendix A for its proof.

\section{EXISTENCE RESULTS}

Our aim is to study the IBVP for equation (2.8) in a bounded domain. Here afterward $U$ is a bounded open connected subset of $\mathbb{R}^{n}, n=2,3, \ldots$ with $C^{2}$ boundary $\Gamma=\partial U$. In this section the number $N \geq 1$, the vectors $\vec{\alpha}$ and $\vec{a}$, and the Forchheimer polynomial $g(s, \vec{a}) \in F P(N, \vec{a})$ all are fixed. Denote $g(s)=g(s, \vec{a})$ and let $K(\xi)$ and $H(\xi)$ be defined as in the previous section.

Consider the following IBVP for $p(x, t)$ :

$$
\left\{\begin{array}{l}
\frac{\partial p}{\partial t}=\nabla \cdot(K(|\nabla p|) \nabla p) \text { in } U \times(0, \infty), \\
p(x, 0)=p_{0}(x) \text { in } U, \\
p(x, t)=\psi(x, t) \text { on } \Gamma \times(0, \infty) .
\end{array}\right.
$$

In order to deal with the non-homogeneous boundary condition, the data $\psi(x, t)$ with $x \in \Gamma$ and $t>0$ is extended to a function $\Psi(x, t)$ with $x \in \bar{U}$ and $t \geq 0$. Throughout, our results are stated in terms of $\Psi$ instead of $\psi$. Nonetheless, corresponding results in terms of $\psi$ can be retrieved as performed in [13].

In this section we prove the global (in time) existence and uniqueness of weak solutions to (3.1). The proof is based on the theory of monotone operators (c.f. [5, 16, 20,23]).

To reduce our problem to the framework of the general theory let us recollect some definitions.

Definition 3.1. Let $\mathbf{V}_{1}, \mathbf{V}_{2}$ be Banach spaces, $A: \mathbf{V}_{1} \rightarrow \mathbf{V}_{2}$ be a map. Then $A$ is called:

- bounded if it maps bounded sets in $\mathrm{V}_{1}$ into bounded sets in $\mathbf{V}_{2}$,

- (weakly) continuous if it maps (weakly) convergent sequences in $\mathbf{V}_{1}$ into (weakly) convergent sequences in $\mathbf{V}_{2}$,

- completely continuous if it maps weakly convergent in $\mathrm{V}_{1}$ sequences into strongly convergent sequences in $\mathbf{V}_{2}$,

- demi-continuous if it maps strongly convergent sequences in $\mathrm{V}_{1}$ into weakly convergent sequences in $\mathbf{V}_{2}$.

In case $\mathbf{V}_{1}=\mathbf{V}$ and $\mathbf{V}_{2}=\mathbf{V}^{\prime}$, that is, $A: \mathbf{V} \rightarrow \mathbf{V}^{\prime}$, the map $A$ is called 
- hemi-continuous if $t \mapsto \mathbf{V}^{\prime}\langle A(u+t v), v\rangle_{\mathbf{V}}$ is continuous for all $u, v \in \mathbf{V}$,

- monotone if $\mathbf{V}^{\prime}\langle A u-A v, u-v\rangle_{\mathbf{v}} \geq 0$ for all $u, v \in \mathbf{V}$,

- of type $\mathbf{M}$ if for every sequence $\left\{u_{n}\right\}_{n=1}^{\infty}$ in $\mathbf{V}$ such that $u_{n} \rightarrow u$ weakly in $\mathbf{V}$, $A u_{n} \rightarrow \xi$ weakly in $\mathbf{V}^{\prime}$ as $n \rightarrow \infty$, and $\limsup _{n \rightarrow \infty} \mathbf{V}^{\prime}\left\langle A u_{n}, u_{n}\right\rangle_{\mathbf{V}} \leq \mathbf{V}^{\prime}\langle\xi, u\rangle_{\mathbf{V}}$, one has $A u=\xi$.

Above, $\mathbf{V}^{\prime}$ denotes the dual space of $\mathbf{V}$ and ${ }_{\mathbf{V}^{\prime}}\langle w, v\rangle_{\mathbf{V}}$ denotes $w(v)$ for $w \in \mathbf{V}^{\prime}$ and $v \in \mathbf{V}$.

From Lemmas 2.1 and 2.2 in [20] follows:

Proposition 3.2. Let $\mathbf{V}$ be a Banach space and let $A: \mathbf{V} \rightarrow \mathbf{V}^{\prime}$. Then:

(i) if $A$ is monotone and hemi-continuous then $A$ is of type $M$,

(ii) if $A$ is bounded and of type $M$ then it is demi-continuous.

Next we prove an abstract existence result which will be applied to our particular problem. This follows Propositions 4.1, 4.2 and 5.1 in Chapter III from [20], and Theorem 30.A from [23] with necessary modifications. We provide some details of the proof for the sake of unity and self-containment. Below, for a number $r>1$ we denote $r^{\prime}$ its conjugate, that is, $1 / r+1 / r^{\prime}=1$.

Theorem 3.3. Let $\mathbf{V}$ be a separable reflexive Banach space, and $\mathbf{H}$ be a separable Hilbert space such that $\mathbf{V} \cap \mathbf{H}$ is dense in $H$. Assume that a family of operators

$$
A(t): \mathbf{V} \rightarrow \mathbf{V}^{\prime}, \quad t \in(0, T),
$$

is given such that

(1) for every $v \in \mathbf{V}$, the map $t \mapsto A(t) v$ is a measurable map $(0, T) \rightarrow \mathbf{V}^{\prime}$,

(2) for almost all (a.a.) $t \in(0, T)$, the operator $A(t)$ is monotone, hemi-continuous and bounded with

$$
\|A(t) v\|_{\mathbf{V}^{\prime}} \leq c\left(\|v\|_{\mathbf{V}}^{r-1}+k_{0}^{r-1}(t)\right), \text { for all } v \in \mathbf{V}
$$

with some constant $c>0$, a number $r \in(1, \infty)$, and a non-negative function $k_{0} \in L^{r}(0, T)$.

(3) there exists a semi-norm $[\cdot]$ on $\mathrm{V}$, constants $\gamma, \lambda>0$, and a non-negative function $k_{1} \in L^{r}(0, T)$ such that

$$
\begin{gathered}
\lambda\|v\|_{\mathbf{H}}+[v] \geq \gamma\|v\|_{\mathbf{V}} \text { for all } v \in \mathbf{V} \cap \mathbf{H}, \\
\mathbf{V}^{\prime}\langle A(t) v, v\rangle_{\mathbf{V}} \geq \gamma[v]^{r}-k_{1}^{r}(t) \text { for all } v \in \mathbf{V} \text {, a.a. } t \in(0, T) .
\end{gathered}
$$

Part I. Then, for every $u_{0} \in \mathbf{H}$ and $f \in L^{r^{\prime}}\left(0, T ; \mathbf{V}^{\prime}\right)$ there exists a solution $u$ to the abstract Cauchy problem

$$
u^{\prime}+A(t) u=f(t) \text { in } L^{r^{\prime}}\left(0, T ; \mathbf{V}^{\prime}\right), \quad u(0)=u_{0},
$$


that satisfies

$$
u \in C([0, T] ; \mathbf{H}) \cap L^{r}(0, T ; \mathbf{V}), u^{\prime} \in L^{r^{\prime}}\left(0, T ; \mathbf{V}^{\prime}\right)
$$

Moreover, this solution is unique in the class

$$
\left\{u \in L^{r}(0, T ; \mathbf{V} \cap \mathbf{H}): u^{\prime} \in L^{r^{\prime}}\left(0, T ; \mathbf{V}^{\prime}+\mathbf{H}\right)\right\}
$$

Part II. In addition, assume that there exists a map $\Phi=\Phi(v, t)$ from $\mathbf{V} \times[0, T]$ to $\mathbb{R}^{+}$, convex and locally bounded in the first variable for all $t \in[0, T]$, such that the following chain rule holds

$$
\frac{d}{d t} \Phi(v(t), t)=\mathbf{v}^{\prime}\left\langle A(t) v(t), v^{\prime}(t)\right\rangle_{\mathbf{v}}+\partial_{t} \Phi(v(t), t) \quad \text { for a.a. } t \in(0, T)
$$

for every absolutely continuous finite dimensional trajectory $v:(0, T) \rightarrow \mathbf{V} \cap \mathbf{H}$ with $v^{\prime} \in L^{r^{\prime}}(0, T ; \mathbf{V})$. Assume there is $c>0$ such that

$$
\left|\partial_{t} \Phi(v, t)\right| \leq c\left(\|v\|_{\mathbf{V}}^{r}+k_{2}(t)\right) \text { for all } v \in \mathbf{V} \cap \mathbf{H}, \text { a.a. } t \in(0, T)
$$

where $k_{2}$ is a non-negative function. Then the following regularity result holds.

(i) If $f \in L^{2}\left(0, T ; \mathbf{H} ; t^{\mu} d t\right)$, $\Phi(0, \cdot) \in L^{1}\left(0, T ; t^{\mu-1} d t\right)$ and $k_{2} \in L^{1}\left(0, T ; t^{\mu} d t\right)$ for some $\mu \geq 1$ then

$$
u^{\prime} \in L^{2}\left(0, T ; H ; t^{\mu} d t\right) \text { and } \operatorname{esssup}_{t \in(0, T)} t^{\mu} \Phi(u(t), t)<\infty
$$

In particular, the identity

$$
u^{\prime}(t)+A(t) u(t)=f(t) \text { holds in } \mathbf{H} \text { for a.a. } t \in(0, T) .
$$

Moreover, $u:(0, T] \rightarrow \mathbf{V}$ is weakly continuous.

(ii) If $f \in L^{2}(0, T ; \mathbf{H}), k_{2} \in L^{1}(0, T)$ and $u_{0} \in \mathbf{V} \cap \mathbf{H}$ then

$$
u^{\prime} \in L^{2}(0, T ; H) \text { and } \operatorname{essup}_{t \in(0, T)} \Phi(u(t), t)<\infty .
$$

Moreover, if in addition $k_{1}$ is bounded in a neighborhood of zero, then $u:[0, T] \rightarrow$ $\mathrm{V}$ is also weakly continuous at $t=0$. 
Remark 3.4. Let $m>0, t>0$ and $v \in \mathbf{V} \cap \mathbf{H}$, we define $v(s)=(s / t)^{m} v$ for $s \in$ $\left[2^{-\frac{1}{m r}} t, t\right]$. It follows from (3.6), (3.7), (3.3) and (3.4) that

$$
\begin{aligned}
\Phi(v, t) \geq & \left.\Phi\left((s / t)^{m} v, s\right)\right|_{s=2^{-\frac{1}{m}} t} ^{s=t} \\
= & \int_{2^{-\frac{1}{m r} t}}^{t}\left[\frac{m}{s} \mathbf{V}^{\prime}\left\langle A(s)(s / t)^{m} v,(s / t)^{m} v\right\rangle_{\mathbf{V}}+\partial_{t} \Phi\left((s / t)^{m} v, s\right)\right] d s \\
\geq & \int_{2^{-\frac{1}{m r}} t}^{t} \gamma \frac{m}{s}\left[(s / t)^{m} v\right]^{r} d s-\int_{2^{-\frac{1}{m r} t}}^{t} \frac{m}{s} k_{1}^{r}(s) d s \\
& -c \int_{2^{-\frac{1}{m r}} t}^{t}\left\|(s / t)^{m} v\right\|_{\mathbf{V}}^{r} d s-c \int_{2^{-\frac{1}{m r}} t}^{t} k_{2}(s) d s \\
= & \frac{\gamma}{r}\left(1-2^{-1}\right)[v]^{r}-\frac{c t}{m r+1}\left(1-2^{-\frac{m r+1}{m r}}\right)\|v\|_{\mathbf{V}}^{r}-\int_{2^{-\frac{1}{m r}} t}^{t}\left(\frac{m}{s} k_{1}^{r}(s)+c k_{2}(s)\right) d s \\
\geq & \left(\frac{\gamma}{2 r}-\frac{2^{r} c t}{m r \gamma^{r}}\right)[v]^{r}-\frac{2^{r} \lambda^{r} c t}{m r \gamma^{r}}\|v\|_{\mathbf{H}}^{r}-\int_{2^{-\frac{1}{m r}} t}^{t}\left(\frac{m}{s} k_{1}^{r}(s)+c k_{2}(s)\right) d s .
\end{aligned}
$$

Hence, choosing $m=2^{r+2} c t / \gamma^{r+1}$, we obtain

$$
\frac{\gamma}{4 r}[v]^{r} \leq \Phi(v, t)+\frac{\gamma \lambda^{r}}{4 r}\|v\|_{\mathbf{H}}^{r}+\int_{2^{-\frac{1}{m r} t}}^{t}\left(\frac{m}{s} k_{1}^{r}(s)+c k_{2}(s)\right) d s .
$$

So holds with

$$
[v]^{r} \leq k_{3}(t)\left(\Phi(v, t)+\|v\|_{\mathbf{H}}^{r}+1\right) \text { for all } v \in \mathbf{V} \cap \mathbf{H} \text { and } t \in(0, T)
$$

where

$$
k_{3}(t)=\frac{4 r}{\gamma}+\lambda^{r}+\frac{4 r}{\gamma} \int_{2^{-\frac{1}{m r} t}}^{t}\left(\frac{m}{s} k_{1}^{r}(s)+c k_{2}(s)\right) d s .
$$

If $k_{2} \in L_{l o c}^{1}((0, T])$, then $k_{3} \in L_{l o c}^{\infty}((0, T])$. Moreover, since $\int_{2^{-\frac{1}{m r}} t}^{t} \frac{m}{s} d s=\frac{\ln 2}{r}$, the above function $k_{3}$ is bounded in a neighborhood of zero provided $k_{1}$ is bounded in a neighborhood of zero and $k_{2} \in L^{1}(0, T)$.

Proof of Theorem 3.3. Note the following.

- By Philips theorem, $L^{r}(0, T ; \mathbf{V})^{\prime} \simeq L^{r^{\prime}}\left(0, T ; \mathbf{V}^{\prime}\right)$.

- By Proposition 3.2, $A(t)$ is demi-continuous for a.a. $t \in(0, T)$. So, given a measurable map $w:(0, T) \rightarrow \mathbf{V}$, and a sequence of simple functions $w_{n}$ converging to $w$ a.e. in $t$ as $n \rightarrow \infty$, one has $A(t) w_{n}(t) \rightarrow A(t) w(t)$ weakly in $\mathbf{V}^{\prime}$ for a.a. $t \in(0, T)$. Hence $t \mapsto A(t) w(t)$ is a measurable map.

- Due to (3.2), the map $\mathcal{A}: L^{r}(0, T ; \mathbf{V}) \rightarrow L^{r^{\prime}}\left(0, T ; \mathbf{V}^{\prime}\right)$ defined by $\mathcal{A} w(t) \stackrel{\text { def }}{=}$ $A(t) w(t)$ is a bounded hemi-continuous map. Moreover, since $A(t)$ is monotone for a.a. $t \in(0, T)$, the map $\mathcal{A}$ is monotone as well. Therefore $\mathcal{A}$ is of type $M$ and demicontinuous.

- The uniqueness of the solution $u$ follows from the fact that $\mathcal{A}$ is a monotone operator.

Case A. First we consider the case $\mathbf{V} \hookrightarrow \mathbf{H}$. 
Part I. Note that in this case $\mathrm{V} \hookrightarrow \mathbf{H} \backsim \mathbf{H}^{\prime} \hookrightarrow \mathbf{V}^{\prime}$, where both embeddings are dense. Since $\mathbf{V}$ is separable, there exists a countable set $F \subset \mathbf{V}$ dense in $\mathbf{V}, \mathbf{H}$ and $\mathbf{V}^{\prime}$. The Gram-Schmidt orthonormalization procedure in $\mathbf{H}$ produces a Schauder basis $\left\{e_{k}\right\}_{k \in \mathbb{N}} \subset \mathbf{V}$ which spans $\mathbf{V}, \mathbf{H}$ and $\mathbf{V}^{\prime}$, being orthonormal in $\mathbf{H}$ and biorthogonal in $\left\langle\mathbf{V}^{\prime}, \mathbf{V}\right\rangle$.

For $n \in \mathbb{N}$, let $\mathbf{V}_{n}=\operatorname{span}\left\{e_{k}: 1 \leq k \leq n\right\}$ and let $P_{n}$ denote the projection $\mathbf{V}^{\prime} \rightarrow \mathbf{V}_{n}$ defined by $P_{n} v \stackrel{\text { def }}{=} \sum_{k=1}^{n} \mathbf{V}^{\prime}\left\langle v, e_{k}\right\rangle_{\mathbf{V}} e_{k}$ for any $v \in \mathbf{V}^{\prime}$.

Let $A_{n}(t)=P_{n} A(t) P_{n}: \mathbf{V}_{n} \rightarrow \mathbf{V}_{n}$ and $f_{n}(t)=P_{n} f(t)$.

Let $u_{0 n} \in \mathbf{V}_{n}$ be such that $u_{0 n} \rightarrow u_{0}$ in $\mathbf{H}$ as $n \rightarrow \infty$.

Consider the following initial value problem in $\mathbf{V}_{n}$ :

$$
u_{n}^{\prime}=A_{n}(t) u_{n}+f_{n}(t), \quad u_{n}(0)=u_{0 n} .
$$

Since $A(t)$ is demi-continuous on $\mathbf{V}$ for a.a. $t \in(0, T)$, it follows that $A_{n}(t)$ is continuous on $\mathbf{V}_{n}$ for a.a. $t \in(0, T)$. Then, by the Cauchy-Peano and Carathéodory theorems, (3.2) implies that (3.9) has a (unique) local solution $u_{n}$, called Galerkin approximation, on $\left[0, T_{n}\right]$ with $0<T_{n} \leq T, u_{n} \in L^{r}\left(0, T_{n} ; \mathbf{V}_{n}\right)$ and $u_{n}^{\prime} \in L^{r^{\prime}}\left(0, T_{n} ; \mathbf{V}_{n}\right)$. Note that this implies $u_{n} \in C\left(\left[0, T_{n}\right], \mathbf{V}_{n}\right)$. The solution $u_{n}$ is extendable to the whole interval $[0, T]$, i.e., $T_{n}=T$, unless it blows up. We derive an estimate of $u_{n}$ which is independent of $n$ to show the absence of a blow up and use it in passing to the limit in $n$.

Multiply (3.9) by $u_{n}$ and integrate in $t$ to obtain

$$
\frac{1}{2}\left\|u_{n}(t)\right\|_{\mathbf{H}}^{2}+\int_{0}^{t} \mathbf{V}^{\prime}\left\langle A(s) u_{n}(s), u_{n}(s)\right\rangle_{\mathbf{V}} d s=\frac{1}{2}\left\|u_{0 n}\right\|_{\mathbf{H}}^{2}+\int_{0}^{t} \mathbf{V}^{\prime}\left\langle f(s), u_{n}(s)\right\rangle_{\mathbf{V}} d s .
$$

Let $k(t)=\max \left\{k_{0}(t), k_{1}(t)\right\}, t \in(0, T)$. Note that, by (3.4)

$$
\int_{0}^{t} \mathbf{V}^{\prime}\left\langle A(s) u_{n}(s), u_{n}(s)\right\rangle_{\mathbf{V}} d s \geq \gamma \int_{0}^{t}\left[u_{n}\right]^{r} d s-\|k\|_{L^{r}(0, T)}^{r}
$$

and, by (3.3) and the Hölder and Young inequalities, there are positive constants $C_{\gamma, r}$ and $C_{\gamma, \lambda, r}$ such that

$$
\begin{array}{r}
\int_{0}^{t} \mathbf{V}^{\prime}\left\langle f(s), u_{n}(s)\right\rangle_{\mathbf{V}} d s \leq \int_{0}^{t}\|f(s)\|_{\mathbf{V}^{\prime}}\left\|u_{n}(s)\right\|_{\mathbf{V}} d s \leq \frac{1}{\gamma} \int_{0}^{t}\|f\|_{\mathbf{V}^{\prime}}\left(\left[u_{n}\right]+\lambda\left\|u_{n}\right\|_{\mathbf{H}}\right) d s \\
\leq C_{\gamma, r}\||f|\|_{r^{\prime}}^{r^{\prime}}+C_{\gamma, \lambda, r}\||f|\|_{r^{\prime}}^{2}+\frac{\gamma}{2} \int_{0}^{t}\left[u_{n}\right]^{r} d s+\frac{1}{2}\left(\int_{0}^{t}\left\|u_{n}\right\|_{\mathbf{H}}^{r} d s\right)^{\frac{2}{r}},
\end{array}
$$

where $\||\cdot|\|_{r^{\prime}}$ denotes the norm in $L^{r^{\prime}}\left(0, T ; \mathbf{V}^{\prime}\right)$. So there exists a constant $c_{\gamma, \lambda, r}>0$ such that

$$
\begin{aligned}
& \left\|u_{n}(t)\right\|_{\mathbf{H}}^{2}+\gamma \int_{0}^{t}\left[u_{n}\right]^{r} d s \\
& \quad \leq\left\|u_{0}\right\|_{H}^{2}+2\|k\|_{L^{r}(0, T)}^{r}+c_{\gamma, \lambda, r}\left(\left\|f\left|\left\|_{r^{\prime}}^{r^{\prime}}+\right\|\|f \mid\|_{r^{\prime}}^{2}\right)+\left(\int_{0}^{t}\left\|u_{n}\right\|_{\mathbf{H}}^{r} d s\right)^{\frac{2}{r}} .\right.\right.
\end{aligned}
$$


In particular, neglecting the integral term on the left-hand side and raising both sides to the power $r / 2$ give

with

$$
\left\|u_{n}(t)\right\|_{\mathbf{H}}^{r} \leq c\left(1+\int_{0}^{t}\left\|u_{n}\right\|_{\mathbf{H}}^{r} d s\right)
$$

$$
c=2^{\frac{r}{2}}\left(1+\left\|u_{0}\right\|_{H}^{2}+2\|k\|_{L^{r}(0, T)}^{r}+c_{\gamma, \lambda, r}\left(\||f|\|_{r^{\prime}}^{r^{\prime}}+\||f|\|_{r^{\prime}}^{2}\right)\right)^{\frac{r}{2}}
$$

independent of $n$. By Gronwall's inequality, $\left\|u_{n}(t)\right\|_{\mathbf{H}}^{r} \leq c e^{c t}$ and hence the solution is extendable to the interval $[0, T]$ and, by (3.10) and (3.3),

$$
\sup _{t \in[0, T]}\left\|u_{n}(t)\right\|_{\mathbf{H}}^{2}+\int_{0}^{T}\left\|u_{n}\right\|_{\mathbf{V}}^{r} d s \text { is bounded uniformly in } n \text {. }
$$

Since $\mathcal{A}$ is a bounded operator $L^{r}(0, T ; \mathbf{V}) \rightarrow L^{r^{\prime}}\left(0, T ; \mathbf{V}^{\prime}\right)$, it follows that $\mathcal{A} u_{n}$ is bounded in $L^{r^{\prime}}\left(0, T ; \mathbf{V}^{\prime}\right)$ uniformly in $n$. Since a closed ball in $L^{r}(0, T ; \mathbf{V})$ is weakly compact, the sequence $\left\{u_{n}\right\}$ has a weak limit point $u \in L^{r}(0, T ; \mathbf{V})$. Let $\left\{u_{n_{k}}\right\}_{k=1}^{\infty}$ be a subsequence such that

$$
u_{n_{k}} \rightarrow u \text { weakly in } L^{r}(0, T ; \mathbf{V})
$$

Since closed balls in $L^{r^{\prime}}\left(0, T ; \mathbf{V}^{\prime}\right)$ and in $\mathbf{H}$ are weakly compact as well, the sequence $\left\{\mathcal{A} u_{n_{k}}\right\}_{k=1}^{\infty}$ has a weak limit point $\xi \in L^{r^{\prime}}\left(0, T ; \mathbf{V}^{\prime}\right)$ and $\left\{u_{n_{k}}(T)\right\}_{k=1}^{\infty}$ has a weak limit point $u^{*} \in \mathbf{H}$. Passing if necessary to a subsequence of $\left\{u_{n_{k}}\right\}_{k=1}^{\infty}$ (which we denote by the same notation), we may assume that

$$
\begin{aligned}
& \mathcal{A} u_{n_{k}} \rightarrow \xi \text { weakly in } L^{r^{\prime}}\left(0, T ; \mathbf{V}^{\prime}\right), \text { and } \\
& u_{n_{k}}(T) \rightarrow u^{*} \text { weakly in } \mathbf{H} \text { as } k \rightarrow \infty
\end{aligned}
$$

Note that

$-\int_{0}^{T} \theta^{\prime}(t)_{\mathbf{V}^{\prime}}\left\langle u_{n_{k}}(t), v\right\rangle_{\mathbf{V}} d t=\int_{0}^{T} \theta(t)_{\mathbf{V}^{\prime}}\left\langle A(t) u_{n_{k}}(t), v\right\rangle_{\mathbf{V}} d t+\int_{0}^{T} \theta(t)_{\mathbf{V}^{\prime}}\langle f(t), v\rangle_{\mathbf{V}} d t$ for all $\theta \in C_{c}^{1}(0, T)$ and $v \in \mathbf{V}_{n_{k}}$. Since $F \stackrel{\text { def }}{=} \cup_{n} \mathbf{V}_{n}$ is dense in $\mathbf{V}$, it follows that

$$
-\int_{0}^{T} \theta^{\prime}(t)_{\mathbf{V}^{\prime}}\langle u(t), v\rangle_{\mathbf{V}} d t=\int_{0}^{T} \theta(t)_{\mathbf{V}^{\prime}}\langle\xi(t), v\rangle_{\mathbf{V}} d t+\int_{0}^{T} \theta(t)_{\mathbf{V}^{\prime}}\langle f(t), v\rangle_{\mathbf{V}} d t
$$

for all $\theta \in C_{c}^{1}(0, T)$ and $v \in \mathbf{V}$. By Proposition 23.20(b) in [23], this implies $u$ is weakly differentiable and

$$
u^{\prime}=-\xi+f \in L^{r^{\prime}}\left(0, T ; \mathbf{V}^{\prime}\right) .
$$

Hence, by Proposition 23.23(ii) in [23], $u \in C([0, T] ; \mathbf{H}), u(T)=u^{*}$ and

$$
\int_{0}^{T} \mathbf{v}^{\prime}\langle\xi(t), u(t)\rangle_{\mathbf{V}} d t=\int_{0}^{T} \mathbf{v}^{\prime}\langle f(t), u(t)\rangle_{\mathbf{v}} d t+\frac{1}{2}\left\|u_{0}\right\|_{H}^{2}-\frac{1}{2}\|u(T)\|_{H}^{2} .
$$

We are left to show that $\mathcal{A} u=\xi$. Since $\mathcal{A}$ is of $M$-type, and one already has (3.12) and (3.13), it suffices to show that

$$
\limsup _{k \rightarrow \infty} \int_{0}^{T} \mathbf{v}^{\prime}\left\langle A(t) u_{n_{k}}(t), u_{n_{k}}(t)\right\rangle_{\mathbf{V}} d t \leq \int_{0}^{T} \mathbf{v}^{\prime}\langle\xi(t), u(t)\rangle_{\mathbf{V}} d t
$$


Indeed, it follows from (3.9) that

$$
\begin{aligned}
\limsup _{k \rightarrow \infty} & \int_{0}^{T} \mathbf{v}^{\prime}\left\langle A(t) u_{n_{k}}(t), u_{n_{k}}(t)\right\rangle_{\mathbf{V}} d t \\
& =\int_{0}^{T} \mathbf{v}^{\prime}\langle f(t), u(t)\rangle_{\mathbf{V}} d t+\frac{1}{2}\left\|u_{0}\right\|_{H}^{2}-\frac{1}{2} \liminf _{k \rightarrow \infty}\left\|u_{n_{k}}(T)\right\|_{H}^{2} \\
& \leq \int_{0}^{T} \mathbf{v}^{\prime}\langle f(t), u(t)\rangle_{\mathbf{V}} d t+\frac{1}{2}\left\|u_{0}\right\|_{H}^{2}-\frac{1}{2}\|u(T)\|_{H}^{2} .
\end{aligned}
$$

The last inequality is due to (3.14) and $\|\cdot\|_{\mathbf{H}}$ being lower semi-continuous in the weak topology. Combining with (3.16), we obtain (3.17). Thus $\mathcal{A} u=\xi$ and (3.15) implies (3.5). So every weak limit point of $\left\{u_{n}\right\}_{n=1}^{\infty}$ is a solution to (3.5). Since such a solution is unique, we conclude that

$$
u_{n} \rightarrow u \text { weakly in } L^{r}(0, T ; \mathbf{V}) \text { as } n \rightarrow \infty \text {. }
$$

Similar argument shows that $\mathcal{A} u$ is the only weak limit point of the sequence $\left\{\mathcal{A} u_{n}\right\}_{n=1}^{\infty}$ in $L^{r^{\prime}}\left(0, T ; \mathbf{V}^{\prime}\right)$. So $\mathcal{A} u_{n} \rightarrow \mathcal{A} u$ weakly in $L^{r^{\prime}}\left(0, T ; \mathbf{V}^{\prime}\right)$ as $n \rightarrow \infty$.

The latter implies that, for all $v \in \mathbf{H}, t \in[0, T]$,

$$
\left|\mathbf{V}^{\prime}\left\langle v, u_{n}(t)-u(t)\right\rangle_{\mathbf{V}}\right| \rightarrow 0 \text { as } n \rightarrow \infty .
$$

Since $\sup _{n \geq 1} \max _{t \in[0, T]}\left\|u_{n}(t)\right\|_{\mathbf{H}}$ and $\max _{t \in[0, T]}\|u(t)\|_{\mathbf{H}}$ are bounded, it suffices to prove (3.19) for $v \in F$. Let $m>1$ and $v \in \mathbf{V}_{m}$. Let $n>m$. Then

$$
\begin{aligned}
& \left|\mathbf{V}^{\prime}\left\langle u_{n}(t)-u(t), v\right\rangle_{\mathbf{V}}\right| \leq\left\|u_{n 0}-u_{0}\right\|_{\mathbf{H}}\|v\|_{\mathbf{H}}+\left|\int_{0}^{t} \mathbf{V}^{\prime}\left\langle u_{n}^{\prime}(\tau)-u^{\prime}(\tau), v\right\rangle_{\mathbf{V}} d \tau\right| \\
& =\left\|u_{n 0}-u_{0}\right\|_{\mathbf{H}}\|v\|_{\mathbf{H}}+\left|\int_{0}^{t} \mathbf{V}^{\prime}\left\langle A(\tau) u_{n}(\tau)-A(\tau) u(\tau), v\right\rangle_{\mathbf{V}} d \tau\right| \\
& \quad+\left|\int_{0}^{t} \mathbf{V}^{\prime}\left\langle f_{n}(\tau)-f(\tau), v\right\rangle_{\mathbf{V}} d \tau\right| \\
& =\left\|u_{n 0}-u_{0}\right\|_{\mathbf{H}}\|v\|_{\mathbf{H}}+\left|\int_{0}^{t} \mathbf{v}^{\prime}\left\langle A(\tau) u_{n}(\tau)-A(\tau) u(\tau), v\right\rangle_{\mathbf{V}} d \tau\right| \rightarrow 0 \text { as } n \rightarrow \infty .
\end{aligned}
$$

(In fact, Theorem 30.A of [23] has much stronger result, namely,

$$
\max _{t \in[0, T]}\left\|u_{n}(t)-u(t)\right\|_{H} \rightarrow 0 \text { as } n \rightarrow \infty .
$$

However, the weak convergence in (3.19) with a short proof adequately serves our purpose at the moment.)

Part II. The proof of this part requires additional estimates of $u_{n}$, independent of $n$.

(i) By (3.6),

$$
\frac{d}{d t} \Phi\left(u_{n}(t), t\right)=\mathbf{v}^{\prime}\left\langle A(t) u_{n}(t), u_{n}^{\prime}(t)\right\rangle_{\mathbf{v}}+\partial_{t} \Phi\left(u_{n}(t), t\right) .
$$


It follows from (3.9) and (3.7) that

$$
\begin{aligned}
& \left\|u_{n}^{\prime}(t)\right\|_{\mathbf{H}}^{2}+\frac{d}{d t} \Phi\left(u_{n}(t), t\right) \leq\left\|u_{n}^{\prime}(t)\right\|_{\mathbf{H}}\|f(t)\|_{\mathbf{H}}+\partial_{t} \Phi\left(u_{n}(t), t\right) \\
& \leq \frac{1}{2}\left\|u_{n}^{\prime}(t)\right\|_{\mathbf{H}}^{2}+\frac{1}{2}\|f(t)\|_{\mathbf{H}}^{2}+C\left(\left\|u_{n}(t)\right\|_{\mathbf{V}}^{r}+k_{2}(t)\right) .
\end{aligned}
$$

Hence

$$
\frac{1}{2}\left\|u_{n}^{\prime}(t)\right\|_{\mathbf{H}}^{2}+\frac{d}{d t} \Phi\left(u_{n}(t), t\right) \leq \frac{1}{2}\|f(t)\|_{\mathbf{H}}^{2}+C\left(\left\|u_{n}(t)\right\|_{\mathbf{V}}^{r}+k_{2}(t)\right) .
$$

Multiply by $t^{\mu}$ and integrate in $t$ to obtain the following:

$$
\begin{aligned}
& \int_{0}^{T} t^{\mu}\left\|u_{n}^{\prime}(t)\right\|_{\mathbf{H}}^{2} d t+\sup _{t \in[0, T]} t^{\mu} \Phi\left(u_{n}(t), t\right) \\
& \leq \frac{3}{2} \int_{0}^{T} t^{\mu}\|f(t)\|_{\mathbf{H}}^{2} d t+\frac{3}{2} \mu \int_{0}^{T} t^{\mu-1} \Phi\left(u_{n}(t), t\right) d t+C T^{\mu}\left\|\left|u_{n}\right|\right\|_{r}^{r}+C \int_{0}^{T} t^{\mu} k_{2}(t) d t
\end{aligned}
$$

here $\left\|\left|u_{n}\right|\right\|_{r}$ stands for the norm of $u_{n}$ in $L^{r}(0, T ; \mathbf{V})$. Now observe that, since $v \mapsto$ $\Phi(v, t)$ is convex for all $t \in[0, T]$, it follows from (3.2) that

$$
\Phi\left(u_{n}(t), t\right) \leq \Phi(0, t)+\mathbf{V}^{\prime}\left\langle A(t) u_{n}, u_{n}\right\rangle_{\mathbf{V}} \leq(c+1)\left\|u_{n}(t)\right\|_{\mathbf{V}}^{r}+k_{1}^{r}(t)+\Phi(0, t) .
$$

Hence there exists a constant $c=c_{\mu, T}>0$ such that

$$
\begin{aligned}
& \int_{0}^{T} t^{\mu}\left\|u_{n}^{\prime}(t)\right\|_{\mathbf{H}}^{2} d t+\sup _{t \in[0, T]} t^{\mu} \Phi\left(u_{n}(t), t\right) \\
& \leq c\left(\int_{0}^{T} t^{\mu}\|f(t)\|_{\mathbf{H}}^{2} d t+\left\|\left|u_{n}\right|\right\|_{r}^{r}+\left\|k_{1}\right\|_{L^{r}(0, T)}^{r}+\int_{0}^{T} t^{\mu-1} \Phi(0, t) d t+\int_{0}^{T} t^{\mu} k_{2}(t) d t\right) .
\end{aligned}
$$

Combining (3.21) with (3.11), we have $\left\{u_{n}^{\prime}\right\}_{n=1}^{\infty}$ is uniformly bounded in $L^{2}\left(0, T ; \mathbf{H} ; t^{\mu} d t\right)$, and hence it is weakly pre-compact in $L^{2}\left(0, T ; \mathbf{H} ; t^{\mu} d t\right)$. Let $w \in L^{2}\left(0, T ; \mathbf{H} ; t^{\mu} d t\right)$ be a weak limit point of $\left\{u_{n}^{\prime}\right\}_{n=1}^{\infty}$ and $\left\{u_{n_{k}}\right\}$ be a subsequence of $\left\{u_{n}\right\}_{n=1}^{\infty}$ such that $u_{n_{k}}^{\prime} \rightarrow w$ weakly in $L^{2}\left(0, T ; \mathbf{H} ; t^{\mu} d t\right)$ as $k \rightarrow \infty$. Then, due to (3.18), for every $\theta \in C_{c}^{1}(0, T)$ and $v \in \mathbf{V}$,

$$
\begin{aligned}
\int_{0}^{T} \theta(t)_{\mathbf{V}^{\prime}}\langle w(t), v\rangle_{\mathbf{V}} d t & =\lim _{k} \int_{0}^{T} \theta(t)_{\mathbf{V}^{\prime}}\left\langle u_{n_{k}}^{\prime}(t), v\right\rangle_{\mathbf{V}} d t \\
& =-\lim _{k} \int_{0}^{T} \theta^{\prime}(t)_{\mathbf{V}^{\prime}}\left\langle u_{n_{k}}(t), v\right\rangle_{\mathbf{V}} d t \\
& =-\int_{0}^{T} \theta^{\prime}(t)_{\mathbf{V}^{\prime}}\langle u(t), v\rangle_{\mathbf{V}} d t
\end{aligned}
$$

So $w=u^{\prime}$, by Proposition 23.20(b) in [23]. Since the weak limit point of $\left\{u_{n}^{\prime}\right\}_{n=1}^{\infty}$ is unique, it follows that $u_{n}^{\prime} \rightarrow u^{\prime}$ weakly in $L^{2}\left(0, T ; \mathbf{H} ; t^{\mu} d t\right)$ as $n \rightarrow \infty$ and $u^{\prime} \in$ $L^{2}\left(0, T ; \mathbf{H} ; t^{\mu} d t\right)$. 
Similarly, we have from (3.21) that

$$
\sup _{n} \sup _{t \in[0, T]} t^{\mu} \Phi\left(u_{n}(t), t\right)<\infty .
$$

Because of (3.12) and $\mathbf{V}$ being separable, by passing to a subsequence we may assume $u_{n_{k}}(t) \rightarrow u(t)$ weakly in $\mathbf{V}$ for a.a. $t \in(0, T)$. Since $\Phi$ is convex in the first variable, it is lower semi-continuous with respect to the weak topology of $\mathbf{V}$. Therefore

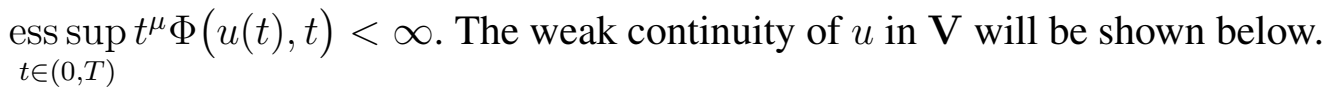

(ii) Note that, since $u_{0} \in \mathbf{V}$, we can choose $u_{n}(0)$ converging to $u_{0}$ in $\mathbf{V}$ rather than in H. Since a locally bounded convex function is continuous, it follows that $\Phi\left(u_{n}(0), 0\right) \rightarrow$ $\Phi\left(u_{0}, 0\right)$ as $n \rightarrow \infty$. Then integration of (3.20) leads to the following:

$$
\begin{aligned}
& \int_{0}^{T}\left\|u_{n}^{\prime}\right\|_{\mathbf{H}}^{2} d t+\sup _{t \in[0, T]} \Phi\left(u_{n}(t), t\right) \\
& \quad \leq c\left(\Phi\left(u_{n}(0), 0\right)+\int_{0}^{T}\|f\|_{\mathbf{H}}^{2} d t+\left\|\left|u_{n}\right|\right\|_{r}^{r}+\int_{0}^{T} k_{2}(t) d t\right) .
\end{aligned}
$$

The argument can be completed as in (i).

Now we prove weak continuity of $u$ in $\mathbf{V}$. It suffices to prove the assertion for $T<\infty$.

Assume (i). By Remark 3.4, we have (3.8) with $k_{3} \in L_{l o c}^{\infty}((0, T])$. Then, by (3.3), (3.22) and (3.11) we have that, for every $\varepsilon>0$,

$$
\left\{u_{n}(t): n \in \mathbb{N}, \varepsilon \leq t \leq T\right\} \text { is bounded in } \mathbf{V} \text { and in } \mathbf{H} \text {. }
$$

For each $t \in[\varepsilon, T]$, property (3.19) implies that $u_{n}(t) \rightarrow u(t)$ weakly in $\mathbf{H}$, hence by the weak pre-compactness in $\mathbf{V}$ and $\mathbf{H}$ of the set in (3.24), we have $u(t) \in \mathbf{V}$. Since $u \in C([0, T] ; \mathbf{H})$, the weak continuity of $u:(0, T] \rightarrow V$ again follows from (3.24).

Assume (ii) with $k_{1}$ bounded in a neighborhood of zero. Then (3.8) holds with $k_{3} \in$ $L^{\infty}(0, T)$. Thanks to (3.23), the boundedness (3.24) holds with $\varepsilon=0$ and the corresponding weak continuity on $[0, T]$ is proved similarly.

Case $B$. Now, consider the general case when $\mathbf{V} \cap \mathbf{H}$ is dense in $\mathbf{H}$. Let $\mathbf{V}_{0}=\mathbf{V} \cap \mathbf{H}$. Then

$$
\mathrm{V}_{0} \hookrightarrow \mathbf{H} \simeq \mathbf{H}^{\prime} \hookrightarrow \mathrm{V}_{0}^{\prime} \simeq \mathbf{V}^{\prime}+\mathbf{H} \text { densely and continuously. }
$$

Moreover, assumptions (1)-(3) hold true with $\mathbf{V}$ and $\mathbf{V}^{\prime}$ replaced with $\mathbf{V}_{0}$ and $\mathbf{V}_{0}^{\prime}$, respectively. Hence problem (3.5) has a unique solution $u$ satisfying

$$
u \in C([0, T] ; \mathbf{H}) \cap L^{r}(0, T ; \mathbf{V} \cap \mathbf{H}), u^{\prime} \in L^{r^{\prime}}\left(0, T ; \mathbf{V}^{\prime}+\mathbf{H}\right) .
$$

However, $C([0, T] ; \mathbf{H}) \cap L^{r}(0, T ; \mathbf{V} \cap \mathbf{H}) \simeq C([0, T] ; \mathbf{H}) \cap L^{r}(0, T ; \mathbf{V})$, and, since $f \in L^{r^{\prime}}\left(0, T ; \mathbf{V}^{\prime}\right)$ and $\mathcal{A}$ is a bounded operator $L^{r}(0, T ; \mathbf{V}) \rightarrow L^{r^{\prime}}\left(0, T ; \mathbf{V}^{\prime}\right)$, we have $u^{\prime}=-\mathcal{A} u+f \in L^{r^{\prime}}\left(0, T ; \mathbf{V}^{\prime}\right)$. The other assertions follow from case A above.

We apply the above abstract result to our problem to obtain the existence of weak solutions globally in time. Below, for any function $\varphi(x, t)$ of two variables $x$ and $t$, we 
use $\varphi(t)$ to denote the function $t \rightarrow \varphi(\cdot, t)$, hence give meaning to expressions such as $\varphi \in C\left((0, T), L^{2}(U)\right)$ and $\|\varphi(t)\|_{L^{2}(U)}$.

Theorem 3.5. (i) For every $p_{0} \in L^{2}(U)$ and

$$
\Psi \in C\left([0, \infty), L^{2}(U)\right) \cap L_{\mathrm{loc}}^{\infty}\left([0, \infty), W^{1,2-a}(U)\right)
$$

with $\Psi_{t} \in L_{\mathrm{loc}}^{(2-a)^{\prime}}\left([0, \infty),\left(W^{1,2-a}(U)\right)^{\prime}\right)$,

the initial boundary value problem (3.1) has a unique solution p satisfying

$$
\begin{aligned}
& \quad p \in C\left([0, \infty), L^{2}(U)\right) \cap L_{\mathrm{loc}}^{\infty}\left([0, \infty), W^{1,2-a}(U)\right) \\
& \text { and } p_{t} \in L_{\mathrm{loc}}^{(2-a)^{\prime}}\left([0, \infty),\left(W^{1,2-a}(U)\right)^{\prime}\right) .
\end{aligned}
$$

(ii) If, in addition, $\Psi:[0, \infty) \rightarrow W^{1,2-a}(U)$ is weakly continuous,

$$
\partial_{t} \Psi \in L_{\mathrm{loc}}^{2}\left([0, \infty), L^{2}(U)\right) \cap L_{\mathrm{loc}}^{2-a}\left([0, \infty), W^{1,2-a}(U)\right),
$$

and $p_{0} \in W^{1,2-a}(U)$ with $\left.p_{0}\right|_{\Gamma}=\left.\Psi(0)\right|_{\Gamma}$ then

$$
p:[0, \infty) \rightarrow W^{1,2-a}(U) \text { is weakly continuous and } \partial_{t} p \in L_{\mathrm{loc}}^{2}\left([0, \infty), L^{2}(U)\right) .
$$

Proof. (i) The uniqueness of a solution $p(x, t)$ is a direct consequence of the monotonicity in Lemma 2.1. For the existence, as usual, we solve for $p(x, t)$ of the form $p(x, t)=$ $\bar{p}(x, t)+\Psi(x, t)$, where $\bar{p}(x, t)$ satisfies

$$
\left\{\begin{array}{l}
\frac{\partial \bar{p}}{\partial t}=\nabla \cdot(K(|\nabla \bar{p}+\nabla \Psi|)(\nabla \bar{p}+\nabla \Psi))-\Psi_{t} \text { in } U \times(0, \infty), \\
\bar{p}(x, t)=0 \text { on } \Gamma \times(0, \infty) .
\end{array}\right.
$$

We will apply Theorem 3.3 to (3.27). Let $\mathbf{V}=\dot{W}^{1,2-a}(U), \mathbf{H}=L^{2}(U)$ and $f=-\partial_{t} \Psi$. Let $T>0$ be arbitrary. Then $f \in L^{(2-a)^{\prime}}\left(0, T ; \mathbf{V}^{\prime}\right)$. For the weak formulation, we multiply the equation by $w \in \mathbf{V}$ and integrate over $U$ to obtain

$$
\frac{d}{d t} \int_{U} \bar{p} w d x=-\int_{U}\left(K(|\nabla \bar{p}+\nabla \Psi(t)|)(\nabla \bar{p}+\nabla \Psi(t)) \cdot \nabla w d x+\int_{U} f w d x .\right.
$$

Therefore we define $A(t) v$, for $t>0$ and $v \in \mathbf{V}$, by

$$
\mathbf{V}^{\prime}\langle A(t) v, w\rangle_{\mathbf{V}}=\int_{U}(K(|\nabla v+\nabla \Psi(t)|)(\nabla v+\nabla \Psi(t)) \cdot \nabla w d x, \quad w \in \mathbf{V} .
$$

Then it follows from (2.10) that

$$
\begin{aligned}
\|A(t) v\|_{\mathbf{V}^{\prime}} & \leq\|K(|\nabla v+\nabla \Psi(t)|)(\nabla v+\nabla \Psi(t))\|_{L^{(2-a)^{\prime}}} \\
& \leq C\|\nabla v+\nabla \Psi(t)\|_{L^{2-a}}^{1-a} \leq C\left(\|v\|_{\mathbf{V}}^{1-a}+\|\nabla \Psi(t)\|_{L^{2-a}}^{1-a}\right) .
\end{aligned}
$$

Hence $A(t)$ is a bounded linear map $\mathbf{V} \rightarrow \mathbf{V}^{\prime}$ and the estimate (3.2) holds with

$$
k_{0}(t)=\|\nabla \Psi(t)\|_{L^{2-a}} \text { and the number } r=2-a .
$$

Obviously, $k_{0} \in L^{r}(0, T)$. Applying (2.10) and the Lebesgue dominated convergence theorem, it is easy to obtain that $A(t)$ is hemi-continuous, $(2.15)$ implies that it is monotone. 
We define the seminorm $[v]$, for $v \in \mathbf{V}$, to be $\|\nabla v\|_{L^{2-a}}$. Then (3.3) clearly holds since $2-a<2$. It follows from estimates (2.10)-(2.11) that, with some $c>0$,

$$
\begin{aligned}
\mathbf{V}^{\prime}\langle A(t) v, v\rangle_{\mathbf{V}} \geq & \int_{U} K(|\nabla v+\nabla \Psi(t)|)|\nabla v+\nabla \Psi(t)|^{2} d x \\
& -\int_{U} K(|\nabla v+\nabla \Psi(t)|)|\nabla v+\nabla \Psi(t)||\nabla \Psi(t)| d x \\
\geq & c\left(\|\nabla v+\nabla \Psi(t)\|_{L^{2-a}}^{2-a}-|U|\right)-\|\nabla v+\nabla \Psi(t)\|_{L^{2-a}}^{1-a}\|\nabla \Psi(t)\|_{L^{2-a}} \\
\geq & \frac{c}{2}\|\nabla v+\nabla \Psi(t)\|_{L^{2-a}}^{2-a}-c|U|-\left(\frac{c}{2}\right)^{a-1}\|\nabla \Psi(t)\|_{L^{2-a}}^{2-a} \\
\geq & c 2^{3-a}\|v\|_{\mathbf{V}}^{2-a}-c|U|-\left(\frac{c}{2}+\left(\frac{c}{2}\right)^{a-1}\right)\|\nabla \Psi(t)\|_{L^{2-a} .}^{2-a}
\end{aligned}
$$

Thus we obtain the coercivity estimate (3.4) with $k_{1}=C\left(1+k_{0}\right)$ for some $C>0$. Applying Theorem 3.3 part I, we obtain solution $\bar{p}(x, t)$ and conclude (3.25) for $p(x, t)$ accordingly.

(ii) Here $f \in L^{2}(0, T ; \mathbf{H})$ and $\bar{p}_{0} \in \mathbf{V} \cap \mathbf{H}$. For $v \in \mathbf{V}$ and $t \geq 0$, let

$$
\Phi(v, t)=\frac{1}{2} \int_{U} H(|\nabla v+\nabla \Psi(t)|) d x .
$$

Then $\Phi(v, t)$ meets the requirements in Theorem 3.3, part II. Indeed, by (2.13),

$$
|\Phi(v, t)| \leq C\left(\|v\|_{\mathbf{V}}^{2-a}+\|\Psi(t)\|_{\mathbf{V}}^{2-a}\right),
$$

hence $\Phi(v, t)$ is bounded in $v$ for each $t \geq 0$.

Let $v, w \in \mathbf{V}$ and $\tau \in(0,1)$, by (2.14) we have

$$
\begin{aligned}
\Phi(\tau v+(1-\tau) w, t) & \leq \frac{1}{2} \int_{U} H(\tau|\nabla v+\nabla \Psi(t)|+(1-\tau)|\nabla w+\nabla \Psi(t)|) d x \\
& \leq \frac{1}{2} \int_{U} \tau H(|\nabla v+\nabla \Psi(t)|)+(1-\tau) H(|\nabla w+\nabla \Psi(t)|) d x \\
& =\tau \Phi(v, t)+(1-\tau) \Phi(w, t) .
\end{aligned}
$$

Therefore $\Phi(v, t)$ is convex in $v$. By direct calculations, one can verify (3.6) and

$$
\partial_{t} \Phi(v, t)=\int_{U} K(|\nabla v+\nabla \Psi(t)|)(\nabla v+\nabla \Psi(t)) \cdot \nabla \partial_{t} \Psi(t) d x .
$$

Then by (2.10),

$$
\begin{aligned}
\left|\partial_{t} \Phi(v, t)\right| & \leq C \int_{U}|\nabla v+\nabla \Psi(t)|^{1-a}\left|\nabla \partial_{t} \Psi(t)\right| d x \\
& \leq C\left(\|v\|_{\mathbf{V}}^{2-a}+\|\nabla \Psi(t)\|_{L^{2-a}}^{2-a}+\left\|\nabla \partial_{t} \Psi(t)\right\|_{L^{2-a}}^{2-a}\right) .
\end{aligned}
$$

Hence (3.7) holds with $k_{2}(t)=\|\nabla \Psi(t)\|_{L^{2-a}}^{2-a}+\left\|\nabla \partial_{t} \Psi(t)\right\|_{L^{2-a}}^{2-a}$. Clearly, $k_{2} \in L^{1}(0, T)$ and $k_{1} \in L^{\infty}(0, T)$. Now the assertion follows from (ii) of part II, Theorem 3.3.

In case the initial and boundary data have more regularity, so does our solution. Specifically, we have: 
Theorem 3.6. Let $\alpha \geq 2$. Assume $p_{0}(x) \in L^{\alpha}(U), \Psi \in C\left([0, \infty) ; L^{\alpha}(U)\right), \nabla \Psi \in$ $L^{\frac{\alpha(2-a)}{2}}(U \times(0, T))$ and $\Psi_{t} \in L^{\alpha}(U \times(0, T))$ for all $T>0$. Then the corresponding solution $p(x, t)$ to (3.1) satisfies $p \in C\left([0, \infty) ; L^{\alpha}(U)\right)$, and $\bar{p}=p-\Psi$ satisfies

$$
\begin{aligned}
\frac{1}{\alpha} \frac{d}{d t} \int_{U}|\bar{p}(x, t)|^{\alpha} d x= & -(\alpha-1) \int_{U} K(|\nabla p|)(\nabla p \cdot \nabla \bar{p})|\bar{p}|^{\alpha-2} d x \\
& +\int_{U} \Psi_{t}|\bar{p}|^{\alpha-2} \bar{p} d x \quad \text { for all } t>0 .
\end{aligned}
$$

Proof. Let $T>0$. For $n \in \mathbb{N}$ and $s \in \mathbb{R}$, let $V_{n}(s)=\int_{0}^{s}(\min \{|\tau|, n\})^{\alpha-2} \tau d \tau$, that is,

$$
V_{n}(s)= \begin{cases}\frac{1}{\alpha}|s|^{\alpha}, & |s| \leq n \\ \frac{1}{\alpha} n^{\alpha}+\frac{1}{2} n^{\alpha-2}(|s|-n)^{2}, & |s|>n .\end{cases}
$$

Clearly, $V_{n}(s) \uparrow \frac{1}{\alpha}|s|^{\alpha}$ as $n \uparrow \infty$. Let also $\bar{p}_{n}=\min \{|\bar{p}|, n\}$. Note that $\bar{p}_{n}^{\alpha-2} \bar{p} \in$ $C\left([0, \infty), W^{1,2-a}(U)\right)$ and that

$$
\nabla\left(\bar{p}_{n}^{\alpha-2} \bar{p}\right)=\left[(\alpha-1) \chi_{n}|\bar{p}|^{\alpha-2}+n^{\alpha-2} \chi_{n}^{c}\right] \nabla \bar{p}
$$

where $\chi_{n}$ is the indicator of the set $\{(x, t):|\bar{p}(x, t)| \leq n\}$ and $\chi_{n}^{c}=1-\chi_{n}$ is the indicator of the complement.

Multiplying the first equation in (3.27) by $\bar{p}_{n}^{\alpha-2} \bar{p}$ and integrating over domain $U$ give

$$
\begin{aligned}
& \frac{d}{d t} \int_{U} V_{n}(\bar{p}) d x=\int_{U} \bar{p}_{n}^{\alpha-2} \bar{p} \frac{\partial \bar{p}}{\partial t} d x \\
& =-\int_{U}\left[K(|\nabla p|)|\nabla p|^{2}-K(|\nabla p|)(\nabla p) \cdot(\nabla \Psi)\right]\left[(\alpha-1) \chi_{n}|\bar{p}|^{\alpha-2}+n^{\alpha-2} \chi_{n}^{c}\right] d x \\
& \quad+\int_{U} \Psi_{t} \bar{p}_{n}^{\alpha-2} \bar{p} d x \equiv I_{1}+I_{2} \cdot
\end{aligned}
$$

We will estimate two integrals on the right-hand side of (3.30).

Note from (2.10)-(2.11) that for $\varepsilon>0$ and $\xi, \eta \geq 0$, we have

$$
K(\xi) \xi \eta \leq C \xi^{1-a} \eta \leq C\left[\left(K(\xi) \xi^{2}\right)^{\frac{1-a}{2-a}}+1\right] \eta \leq \varepsilon K(\xi) \xi^{2}+C_{\varepsilon}\left(\eta^{2-a}+1\right) .
$$

Estimating $K(|\nabla p|)(\nabla p \cdot \nabla \Psi)$ by (3.31) with $\varepsilon=\frac{1}{2}$, there exists $C>0$ such that

$$
\begin{aligned}
-\left[K(|\nabla p|)|\nabla p|^{2}-K(|\nabla p|)(\nabla p) \cdot(\nabla \Psi)\right] & \leq-K(|\nabla p|)|\nabla p|^{2}+K(|\nabla p|)|\nabla p||\nabla \Psi| \\
& \leq-\frac{1}{2} K(|\nabla p|)|\nabla p|^{2}+C\left(|\nabla \Psi|^{2-a}+1\right) .
\end{aligned}
$$

Moreover,

$$
(\alpha-1) \chi_{n}|\bar{p}|^{\alpha-2}+n^{\alpha-2} \chi_{n}^{c} \leq \alpha^{\frac{2 \alpha-2}{\alpha}} V_{n}(\bar{p})^{\frac{\alpha-2}{\alpha}},
$$

and, since $\alpha \geq 2$, we have $p_{n}^{\alpha-2}|\bar{p}| \leq C_{\alpha} V_{n}(\bar{p})^{\frac{\alpha-1}{\alpha}}$. Then, by the Young inequality,

$$
\begin{aligned}
& I_{1} \leq-\frac{1}{2} \alpha^{\frac{2 \alpha-2}{\alpha}} \int_{U} K(|\nabla p|)|\nabla p|^{2} V_{n}(\bar{p})^{\frac{\alpha-2}{\alpha}} d x+C\left(\int_{U} V_{n}(\bar{p}) d x+\int_{U}|\nabla \Psi|^{\frac{\alpha(2-a)}{2}} d x+1\right), \\
& I_{2} \leq \int_{U} V_{n}(\bar{p}) d x+\int_{U}\left|\Psi_{t}\right|^{\alpha} d x .
\end{aligned}
$$


Hence it follows from (3.30) and the above estimate of $I_{1}$ and $I_{2}$ that

$$
\begin{aligned}
\frac{d}{d t} \int_{U} V_{n}(\bar{p}) d x & +\frac{1}{2} \alpha^{\frac{2 \alpha-2}{\alpha}} \int_{U} K(|\nabla p|)|\nabla p|^{2} V_{n}(\bar{p})^{\frac{\alpha-2}{\alpha}} d x \\
& \leq C\left(\int_{U} V_{n}(\bar{p}) d x+\int_{U}|\nabla \Psi|^{\frac{\alpha(2-a)}{2}} d x+\int_{U}\left|\Psi_{t}\right|^{\alpha} d x+1\right) .
\end{aligned}
$$

Drop the second summand on the left-hand side to obtain

$$
\frac{d}{d t} \int_{U} V_{n}(\bar{p}) d x \leq C \int_{U} V_{n}(\bar{p}) d x+C\left(\int_{U}|\nabla \Psi|^{\frac{\alpha(2-a)}{2}} d x+\int_{U}\left|\Psi_{t}\right|^{\alpha} d x+1\right) .
$$

Then it follows from the Gronwall inequality that there exists $C=C(T)>0$ such that

$$
\int_{U} V_{n}(\bar{p}) d x \leq C e^{C t}, \quad 0<t<T .
$$

Passing $n \rightarrow \infty$ and by the Beppo Levi lemma, we have

$$
\int_{U}|\bar{p}|^{\alpha} d x \leq \alpha C e^{C t}, \quad 0<t<T
$$

Therefore $\bar{p} \in L^{\infty}\left(0, T ; L^{\alpha}(U)\right)$.

Now integrate (3.32) in $t$ to obtain that, for all $T>0$,

$$
\begin{aligned}
\int_{0}^{T} \int_{U} K(|\nabla p|)|\nabla p|^{2} V_{n}(\bar{p})^{\frac{\alpha-2}{\alpha}} d x d t \leq & C\left(\int_{0}^{T} \int_{U}|\bar{p}|^{\alpha} d x d t+\int_{0}^{T} \int_{U}|\nabla \Psi|^{\frac{\alpha(2-a)}{2}} d x d t\right. \\
& \left.+\int_{0}^{T} \int_{U}\left|\Psi_{t}\right|^{\alpha} d x d t+\int_{U}\left|\bar{p}_{0}\right|^{\alpha} d x+1\right) .
\end{aligned}
$$

Again, it follows from the Beppo Levi lemma that

$$
K(|\nabla p|)|\nabla p|^{2}|\bar{p}|^{\alpha-2} \in L^{1}(U \times(0, T)) \text { for all } T>0 .
$$

Now (3.29) follows from (3.30) as $n \rightarrow \infty$.

To prove that $\bar{p}(t)$ is continuous in $L^{\alpha}(U)$, observe that it is continuous in measure convergence since it is continuous in $L^{2}(U)$. Then by the Brezis-Lieb lemma [6], it suffices to prove that $\|\bar{p}(t)\|_{L^{\alpha}}$ is continuous. However, by the asserted identity (3.29), similar estimates to those of $I_{1}$ and $I_{2}$, and properties (3.33) and (3.34), it follows that $\int_{0}^{T}\left|\frac{d}{d t}\|\bar{p}(t)\|_{L^{\alpha}}^{\alpha}\right| d t<\infty$, hence $\|\bar{p}(t)\|_{\alpha}^{\alpha}$ is absolutely continuous. Consequently the assertion follows.

\section{Estimates OF SOLUTIONS}

In this section, we obtain more refined estimates for solutions of (3.1) than those in the previous section. We emphasize on the asymptotic estimates as $t \rightarrow \infty$ in terms of the asymptotic behavior of the Dirichlet data. They are crucial to the stability analysis of the solutions in the next two sections.

In previous section, we prove that (3.1) possesses a weak solution $p(x, t)$ for all $t>0$ (see Theorems 3.5 and 3.6). Our solution $p(x, t)$, in fact, has more regularity in spatial and time variables. However, a proof of this fact requires another lengthy treatment which 
is beyond the scope of the current paper. Also, since we aim is to study the qualitative properties of the solutions, we take the liberty to assume that $p(x, t)$ has sufficient regularities both in $x$ and $t$ variables such that our calculations hereafterward can be performed legitimately.

Let $p(x, t)$ be a solution to IBVP (3.1) with given data $p_{0}(x)$ and $\psi(x, t)$. Let $\bar{p}=p-\Psi$, then it satisfies

$$
\left\{\begin{array}{l}
\frac{\partial \bar{p}}{\partial t}=\nabla \cdot(K(|\nabla p|) \nabla p)-\Psi_{t} \text { in } U \times(0, \infty), \\
\bar{p}(x, t)=0 \text { on } \Gamma \times(0, \infty) .
\end{array}\right.
$$

We will derive estimates for $\int_{U}|\bar{p}(x, t)|^{\alpha} d x$ for all $\alpha>0$ and $t>0$, as well as for $\int_{U}|\nabla p(x, t)|^{2-a} d x$ and $\int_{U}\left|\bar{p}_{t}(x, t)\right|^{2} d x$. The corresponding estimates for $p(x, t)$ can be obtained by using the facts

$$
\int_{U}|p|^{\alpha} d x \leq 2^{\alpha} \int_{U}|\bar{p}|^{\alpha}+|\Psi|^{\alpha} d x \text { and } \int_{U}\left|p_{t}\right|^{2} d x \leq 2 \int_{U}\left|\bar{p}_{t}\right|^{2}+\left|\Psi_{t}\right|^{2} d x .
$$

Notation for constants. In this section all constants $C, C_{1}, C_{2}, \ldots$ depend on many parameters, namely, exponent $\alpha$, vectors $\vec{\alpha}$ and $\vec{a}$, the spatial dimension $n$ and domain $U$, but are independent of the initial and boundary data. The constant $C$ is generic and may vary from place to place, even changes values on the same line. Constants $C_{1}, C_{2}, \ldots$ have values temporarily fixed within one proof. The constants $c_{1}, c_{2}, \ldots$, have fixed values for each $\alpha$. Their dependence on $n, U, \vec{\alpha}$ and $\vec{a}$ is implicitly understood. The constants $d_{1}, d_{2}, \ldots$, do not depend on $\alpha$.

The Lebesgue norms (on $U$ ) of $\Psi(x, t)$ or its spatial and time derivatives are always assumed to be continuous in $t$ on $[0, \infty)$ whenever they are used.

For $\alpha>0$ and $t \geq 0$, we define

$$
A(\alpha, t)=A[\Psi](\alpha, t) \stackrel{\text { def }}{=}\left[\int_{U}|\nabla \Psi(x, t)|^{\frac{\alpha(2-a)}{2}} d x\right]^{\frac{2(\alpha-a)}{\alpha(2-a)}}+\left[\int_{U}\left|\Psi_{t}(x, t)\right|^{\alpha} d x\right]^{\frac{\alpha-a}{\alpha(1-a)}}
$$

Lemma 4.1. Suppose $\alpha \geq \max \left\{2, \alpha_{*}\right\}$. Then there exist positive constants $c_{5}=c_{5}(\alpha)$ and $C$ such that

$$
\frac{d}{d t} \int_{U}|\bar{p}(x, t)|^{\alpha} d x \leq-c_{5}\left(\int_{U}|\bar{p}(x, t)|^{\alpha} d x\right)^{\frac{1}{\gamma_{0}}}+C(1+A(\alpha, t)), \quad t>0,
$$

where $\gamma_{0}=\gamma_{0}(\alpha)=\alpha /(\alpha-a)$. 
Proof. Multiplying both sides of the first equation in (4.1) by $|\bar{p}|^{\alpha-1} \operatorname{sign}(\bar{p})$, integrating over domain $U$, and using Green' formula and the facts $\alpha>1, \bar{p}=0$ on $\Gamma$, we have

$$
\begin{aligned}
& \frac{1}{\alpha} \frac{d}{d t} \int_{U}|\bar{p}|^{\alpha} d x=\int_{U}|\bar{p}|^{\alpha-1} \operatorname{sign}(\bar{p}) \frac{\partial \bar{p}}{\partial t} d x \\
= & \int_{U}\left[\nabla \cdot(K(|\nabla p|) \nabla p)-\Psi_{t}\right]|\bar{p}|^{\alpha-1} \operatorname{sign}(\bar{p}) d x \\
= & -(\alpha-1)\left[\int_{U} K(|\nabla p|)|\nabla p|^{2}|\bar{p}|^{\alpha-2} d x-\int_{U} K(|\nabla p|)(\nabla p \cdot \nabla \Psi)|\bar{p}|^{\alpha-2} d x\right] \\
& -\int_{U} \Psi_{t}|\bar{p}|^{\alpha-1} \operatorname{sign}(\bar{p}) d x
\end{aligned}
$$

We will estimate the last three integrals of (4.4). Let $\varepsilon>0$. By (2.11) and Hölder's inequality, we have

$$
\begin{aligned}
& -\int_{U} K(|\nabla p|)|\nabla p|^{2}|\bar{p}|^{\alpha-2} d x \leq-C \int_{U}\left(|\nabla p|^{2-a}-1\right)|\bar{p}|^{\alpha-2} d x \\
& \leq-C \int_{U}|\nabla \bar{p}|^{2-a}|\bar{p}|^{\alpha-2} d x+C \int_{U}|\nabla \Psi|^{2-a}|\bar{p}|^{\alpha-2}+|\bar{p}|^{\alpha-2} d x \\
& \leq-C \int_{U}|\nabla \bar{p}|^{2-a}|\bar{p}|^{\alpha-2} d x+C\left(\int_{U}|\bar{p}|^{\alpha} d x\right)^{\frac{\alpha-2}{\alpha}}\left(\int_{U}\left(1+|\nabla \Psi|^{2-a}\right)^{\frac{\alpha}{2}} d x\right)^{\frac{2}{\alpha}} .
\end{aligned}
$$

Since $\frac{\alpha-2}{\alpha}<\frac{\alpha-a}{\alpha}=\frac{1}{\gamma_{0}}$, applying Young's inequality to the last product gives

$$
\begin{aligned}
-\int_{U} K(|\nabla p|)|\nabla p|^{2}|\bar{p}|^{\alpha-2} d x \leq & -C \int_{U}|\nabla \bar{p}|^{2-a}|\bar{p}|^{\alpha-2} d x+\varepsilon\left(\int_{U}|\bar{p}|^{\alpha} d x\right)^{\frac{1}{\gamma_{0}}} \\
& +C_{\varepsilon}\left[1+\left(\int_{U}|\nabla \Psi|^{\frac{\alpha(2-a)}{2}} d x\right)^{\frac{2(\alpha-a)}{\alpha(2-a)}}\right] .
\end{aligned}
$$

For the second integral on the right-hand side of (4.4),

$$
\begin{aligned}
& \left.\left.\left|\int_{U} K(|\nabla p|)(\nabla p \cdot \nabla \Psi)\right| \bar{p}\right|^{\alpha-2} d x\left|\leq C \int_{U}\right| \nabla p\right|^{1-a}|\nabla \Psi||\bar{p}|^{\alpha-2} d x \\
& \leq C \int_{U}|\nabla \bar{p}|^{1-a}|\nabla \Psi||\bar{p}|^{\alpha-2} d x+\int_{U}|\nabla \Psi|^{2-a}|\bar{p}|^{\alpha-2} d x .
\end{aligned}
$$

Applying Hölder's inequality to functions $|\bar{p}|^{\alpha-2}$ and $|\nabla \Psi|^{2-a}$ using powers $\frac{\alpha}{\alpha-2}$ and $\frac{\alpha}{2}$, and then applying Young's inequality we obtain

$$
\begin{aligned}
\int_{U}|\bar{p}|^{\alpha-2}|\nabla \Psi|^{2-a} d x & \leq\left(\int_{U}|\bar{p}|^{\alpha} d x\right)^{\frac{\alpha-2}{\alpha}}\left(\int_{U}|\nabla \Psi|^{\frac{\alpha(2-a)}{2}} d x\right)^{\frac{2}{\alpha}} \\
& \leq \varepsilon\left(\int_{U}|\bar{p}|^{\alpha} d x\right)^{\frac{1}{\gamma_{0}}}+C_{\varepsilon}\left(\int_{U}|\nabla \Psi|^{\frac{\alpha(2-a)}{2}} d x\right)^{\frac{2(\alpha-a)}{\alpha(2-a)}}
\end{aligned}
$$


Applying Hölder's inequality to three functions $|\nabla \bar{p}|^{1-a}|\bar{p}|^{\frac{(\alpha-2)(1-a)}{2-a}},|\bar{p}|^{\frac{\alpha-2}{2-a}},|\nabla \Psi|$ with powers $\frac{2-a}{1-a}, \frac{\alpha(2-a)}{\alpha-2}, \frac{\alpha(2-a)}{2}$ respectively, we have

$$
\begin{aligned}
& \int_{U}|\nabla \bar{p}|^{1-a}|\nabla \Psi||\bar{p}|^{\alpha-2} d x=\int_{U}|\nabla \bar{p}|^{1-a}|\bar{p}|^{\frac{(\alpha-2)(1-a)}{(2-a)}} \cdot|\bar{p}|^{\frac{\alpha-2}{2-a}} \cdot|\nabla \Psi| d x \\
& \leq\left(\int_{U}|\nabla \bar{p}|^{2-a}|\bar{p}|^{\alpha-2} d x\right)^{\frac{1-a}{2-a}}\left(\int_{U}|\bar{p}|^{\alpha} d x\right)^{\frac{\alpha-2}{\alpha(2-a)}}\left(\int_{U}|\nabla \Psi|^{\frac{\alpha(2-a)}{2}} d x\right)^{\frac{2}{\alpha(2-a)}} .
\end{aligned}
$$

Since $\frac{1-a}{2-a}<1$ and $\frac{\alpha-2}{\alpha(2-a)} \leq \frac{\alpha-2}{\alpha}<\frac{1}{\gamma_{0}}$, applying Young's inequality gives

$$
\begin{aligned}
& \int_{U}|\nabla \bar{p}|^{1-a}|\nabla \Psi||\bar{p}|^{\alpha-2} d x \\
& \leq \varepsilon \int_{U}|\nabla \bar{p}|^{2-a}|\bar{p}|^{\alpha-2} d x+\varepsilon\left(\int_{U}|\bar{p}|^{\alpha} d x\right)^{\frac{1}{\gamma_{0}}}+C_{\varepsilon}\left(\int_{U}|\nabla \Psi|^{\frac{\alpha(2-a)}{2}} d x\right)^{\frac{2(\alpha-a)}{\alpha(2-a)}} .
\end{aligned}
$$

From (4.6), (4.7) and (4.8), we have

$$
\begin{aligned}
\left.\left|\int_{U} K(|\nabla p|) \nabla p \cdot \nabla \Psi\right| \bar{p}\right|^{\alpha-2} d x \mid \leq & \varepsilon \int_{U}|\nabla \bar{p}|^{2-a}|\bar{p}|^{\alpha-2} d x+\varepsilon\left(\int_{U}|\bar{p}|^{\alpha} d x\right)^{\frac{1}{\gamma_{0}}} \\
& +C_{\varepsilon}\left(\int_{U}|\nabla \Psi|^{\frac{\alpha(2-a)}{2}} d x\right)^{\frac{2(\alpha-a)}{\alpha(2-a)}} .
\end{aligned}
$$

For the last integral on the right-hand side of (4.4), by Höder's and Young's inequalities, we similarly obtain

$$
\begin{aligned}
\left.\left|\int_{U} \Psi_{t}\right| \bar{p}\right|^{\alpha-1} \operatorname{sign}(\bar{p}) d x \mid & \leq\left(\int_{U}|\bar{p}|^{\alpha} d x\right)^{\frac{\alpha-1}{\alpha}}\left(\int_{U}\left|\Psi_{t}\right|^{\alpha} d x\right)^{\frac{1}{\alpha}} \\
& \leq \varepsilon\left(\int_{U}|\bar{p}|^{\alpha} d x\right)^{\frac{1}{\gamma_{0}}}+C_{\varepsilon}\left(\int_{U}\left|\Psi_{t}\right|^{\alpha} d x\right)^{\frac{\alpha-a}{\alpha(1-a)}} .
\end{aligned}
$$

Using estimates (4.5), (4.9) and (4.10) in (4.4), we have

$$
\begin{aligned}
\frac{d}{d t} \int_{U}|\bar{p}|^{\alpha} d x \leq & -C \int_{U}|\nabla \bar{p}|^{2-a}|\bar{p}|^{\alpha-2} d x+\varepsilon\left(\int_{U}|\bar{p}|^{\alpha} d x\right)^{\frac{1}{\gamma_{0}}}+\varepsilon \int_{U}|\nabla \bar{p}|^{2-a}|\bar{p}|^{\alpha-2} d x \\
& +C_{\varepsilon}(1+A(\alpha, t)) .
\end{aligned}
$$

For sufficiently small $\varepsilon$, we obtain

$$
\frac{d}{d t} \int_{U}|\bar{p}|^{\alpha} d x \leq-C \int_{U}|\nabla \bar{p}|^{2-a}|\bar{p}|^{\alpha-2} d x+\varepsilon\left(\int_{U}|\bar{p}|^{\alpha} d x\right)^{\frac{1}{\gamma_{0}}}+C_{\varepsilon}(1+A(\alpha, t)) .
$$

Applying inequality (2.19) of Lemma 2.3 to $\int_{U}|\nabla \bar{p}|^{2-a}|\bar{p}|^{\alpha-2} d x$ in (4.11) yields

$$
\frac{d}{d t} \int_{U}|\bar{p}|^{\alpha} d x \leq-C\left(\left(\int_{U}|\bar{p}|^{\alpha} d x\right)^{\frac{1}{\gamma_{0}}}-1\right)+\varepsilon\left(\int_{U}|\bar{p}|^{\alpha} d x\right)^{\frac{1}{\gamma_{0}}}+C_{\varepsilon}(1+A(\alpha, t)) .
$$

Now selecting $\varepsilon$ sufficiently small, we obtain (4.3).

We can estimate the $L^{\alpha}$-norm of $\bar{p}$ for all $\alpha>0$. 
Definition 4.2. For any $\alpha>0$, we define

$$
\widehat{\alpha}=\max \left\{\alpha, 2, \alpha_{*}\right\}= \begin{cases}\max \left\{\alpha, \alpha_{*}\right\} & \text { in the NDC case } \\ \max \{\alpha, 2\} & \text { in the DC case. }\end{cases}
$$

For $\alpha \geq 1$, let

$$
A(\alpha)=\limsup _{t \rightarrow \infty} A(\alpha, t) \text { and } \beta(\alpha)=\limsup _{t \rightarrow \infty}\left[A^{\prime}(\alpha, t)\right]^{-} .
$$

Whenever $\beta(\alpha)$ is in use, it is understood that the function $t \rightarrow A(\alpha, t)$ belongs to $C^{1}((0, \infty))$.

Theorem 4.3. Let $\alpha>0$.

(i) For all $t \geq 0$,

$$
\int_{U}|\bar{p}(x, t)|^{\alpha} d x \leq C\left(1+\int_{U}|\bar{p}(x, 0)|^{\widehat{\alpha}} d x+[\operatorname{Env} A(\widehat{\alpha}, t)]^{\frac{\widehat{\alpha}}{\widehat{\alpha}-a}}\right) .
$$

(ii) If $A(\widehat{\alpha})<\infty$ then

$$
\limsup _{t \rightarrow \infty} \int_{U}|\bar{p}(x, t)|^{\alpha} d x \leq C\left(1+A(\widehat{\alpha})^{\frac{\widehat{\alpha}}{\hat{\alpha}-a}}\right) .
$$

(iii) If $\beta(\widehat{\alpha})<\infty$ then there is $T>0$ such that

$$
\int_{U}|\bar{p}(x, t)|^{\alpha} d x \leq C\left(1+\beta(\widehat{\alpha})^{\frac{\widehat{\alpha}}{\widehat{\alpha}-2 a}}+A(\widehat{\alpha}, t)^{\frac{\widehat{\alpha}}{\widehat{\alpha}-a}}\right)
$$

for all $t \geq T$.

Proof. Since $\alpha \leq \widehat{\alpha}$, by Young's inequality

$$
\int_{U}|\bar{p}(x, t)|^{\alpha} d x \leq \int_{U} 1+|\bar{p}(x, t)|^{\widehat{\alpha}} d x
$$

hence it suffices to prove (4.13), (4.14) and (4.15) for the case $\alpha=\widehat{\alpha}$.

Consider $\alpha=\widehat{\alpha}$. Let $y(t)=\int_{U}|\bar{p}(x, t)|^{\alpha} d x$. Then by (4.3),

$$
y^{\prime}(t) \leq-c_{5} y(t)^{\frac{1}{\gamma_{0}}}+C(1+A(\alpha, t)), \quad t>0 .
$$

(i) Applying estimate (2.33) Lemma 2.7 to inequality (4.16), we obtain

$$
\int_{U}|\bar{p}(x, t)|^{\alpha} d x \leq \int_{U}|\bar{p}(x, 0)|^{\alpha} d x+C\left(1+[\operatorname{Env} A(\alpha, t)]^{\gamma_{0}}\right)
$$

hence (4.13) follows.

(ii) Assume $A(\alpha)<\infty$. Applying (2.34) of Lemma 2.7 to (4.16) yields

$$
\limsup _{t \rightarrow \infty} \int_{U}|\bar{p}(x, t)|^{\alpha} d x \leq C(1+A(\alpha))^{\gamma_{0}}
$$

hence we obtain (4.14).

(iii) Assume $\beta(\alpha)<\infty$. Let $\phi(z)=c_{5}^{-\gamma_{0}} z^{\gamma_{0}}$ for $z \geq 0$. Then $\phi(z) \leq \varphi_{c, \gamma_{0}}(z)$, where $c=c_{5}^{-\gamma_{0}}$, and $\phi^{-1}(z) \geq \varphi_{c, \gamma_{0}}^{-1}(z)$, and it follows from (4.16) that

$$
y^{\prime}(t) \leq-\phi^{-1}(y(t))+C(1+A(\alpha, t)) \leq-\varphi_{c, \gamma_{0}}^{-1}(y(t))+C(1+A(\alpha, t)), \quad t>0 .
$$


Note that $1<\gamma_{0}<2$, applying Proposition 3.7 in [14] to (4.17), there is $T>0$ such that for all $t>T$,

$$
\int_{U}|\bar{p}(x, t)|^{\alpha} d x \leq C\left(1+\beta(\alpha)^{\frac{\gamma_{0}}{2-\gamma_{0}}}+A(\alpha, t)^{\gamma_{0}}\right),
$$

which yields (4.15).

For further estimates of spatial and time derivatives of the pressure, we recall the following quantities from $[13,14]$ :

$$
\begin{aligned}
G_{1}(t)=G_{1}[\Psi](t)= & \int_{U}|\nabla \Psi(x, t)|^{2} d x+\left[\int_{U}\left|\Psi_{t}(x, t)\right|^{r_{0}} d x\right]^{\frac{2-a}{r_{0}(1-a)}} \\
& +\left[\int_{U}\left|\Psi_{t}(x, t)\right|^{r_{0}} d x\right]^{\frac{1}{r_{0}}} \\
G_{2}(t)=G_{2}[\Psi](t)= & \int_{U}\left|\nabla \Psi_{t}(x, t)\right|^{2} d x+\int_{U}\left|\Psi_{t}(x, t)\right|^{2} d x \\
G_{3}(t)=G_{3}[\Psi](t)= & G_{1}(t)+G_{2}(t), \\
G_{4}(t)=G_{4}[\Psi](t)= & G_{3}(t)+\int_{U}\left|\Psi_{t t}\right|^{2} d x
\end{aligned}
$$

where $r_{0}$ is the same as in section 2, or explicitly, $r_{0}=\frac{n(2-a)}{(2-a)(n+1)-n}$.

Using the above $L^{\alpha}$-estimates, we re-estimate and improve the bounds of the integrals $\int_{U} H(|\nabla p|) d x$ and $\int_{U}\left|\bar{p}_{t}\right|^{2} d x$ obtained in [13]. Particularly, our new estimates are much sharper in the NDC case.

From estimate (3.25) in [13] we have for all $t \geq 0$ that

$$
\int_{U} H(|\nabla p(x, t)|) d x \leq \int_{U} H(|\nabla p(x, 0)|)+|\bar{p}(x, 0)|^{2} d x+C \int_{0}^{t} G_{3}(\tau) d \tau .
$$

However, this does not imply the uniform boundedness of $\int_{U} H(|\nabla p(x, t)|) d x$ for $t \geq 0$ even when $G_{3}(t)$ are uniformly bounded.

We easily obtain from inequality (3.4) in [13] that

$$
\int_{0}^{t} \int_{U} H(|\nabla p(x, t)|) d x \leq C \int_{U}|\bar{p}(x, 0)|^{2} d x+C \int_{0}^{t} G_{1}(\tau) d \tau .
$$

Also, we recall (3.25) of [13]:

$$
\int_{0}^{t} \int_{U}\left|\bar{p}_{t}(x, \tau)\right|^{2} d x d \tau \leq \int_{U} H(|\nabla p(x, 0)|)+|\bar{p}(x, 0)|^{2} d x+C \int_{0}^{t} G_{3}(\tau) d \tau .
$$

Because of estimate (4.13), we define for $\alpha>0$ and $t \geq 0$,

$$
B(\alpha, t)=B[\bar{p}(\cdot, 0), \Psi](\alpha, t) \stackrel{\text { def }}{=} \int_{U}|\bar{p}(x, 0)|^{\alpha} d x+[\operatorname{Env} A(\alpha, t)]^{\frac{\alpha}{\alpha-a}} .
$$


Theorem 4.4. (i) If $t \geq 0$ then

$$
\begin{aligned}
\int_{U} H(|\nabla p(x, t)|) d x \leq & C\left(1+\int_{U}|\bar{p}(x, 0)|^{\widehat{2}} d x\right)+e^{-d_{5} t} \int_{U} H(|\nabla p(x, 0)|) d x \\
& +C \int_{0}^{t} e^{-d_{5}(t-\tau)}\left[\left[\operatorname{Env}(A(\widehat{2}, \tau)]^{\frac{\widehat{2}}{2-a}}+G_{3}(\tau)\right] d \tau\right.
\end{aligned}
$$

where $d_{5}>0$ is introduced in (4.26) below.

(ii) If $A(\widehat{2})<\infty$ then there is $T>0$ such that for $t>T$,

$$
\int_{U} H(|\nabla p(x, t)|) d x \leq C\left(1+A(\widehat{2})^{\frac{\widehat{2}}{2-a}}+\int_{0}^{t} e^{-d_{5}(t-\tau)} G_{3}(\tau) d \tau\right)
$$

consequently

$$
\limsup _{t \rightarrow \infty} \int_{U} H(|\nabla p(x, t)|) d x \leq C\left[1+A(\widehat{2})^{\frac{\widehat{2}}{\hat{2}-a}}+\limsup _{t \rightarrow \infty} G_{3}(t)\right] .
$$

(iii) If $\beta(\widehat{2})<\infty$ then there is $T>0$ such that

$$
\int_{U} H(|\nabla p(x, t)|) d x \leq C\left\{1+\beta(\widehat{2})^{\frac{\widehat{2}}{2-2 a}}+\int_{0}^{t} e^{-d_{5}(t-\tau)}\left[A(\widehat{2}, \tau)^{\frac{\widehat{2}}{2-a}}+G_{3}(\tau)\right] d \tau\right\}
$$

for all $t>T$.

Proof. (i) From line 4 into the proof of Corollary 3.7 in [13], we have

$$
\frac{d}{d t} \int_{U} H(|\nabla p|) d x+\frac{1}{2} \int_{U}\left|\bar{p}_{t}\right|^{2} d x \leq-d_{5} \int_{U} H(|\nabla p|) d x+C \int_{U} \bar{p}^{2} d x+C G_{3}(t),
$$

hence neglecting the non-negative term on the left-hand side gives

$$
\frac{d}{d t} \int_{U} H(|\nabla p|) d x \leq-d_{5} \int_{U} H(|\nabla p|) d x+C \int_{U} \bar{p}^{2} d x+C G_{3}(t) .
$$

Applying Gronwall's inequality yields

$$
\begin{array}{rl}
\int_{U} H(|\nabla p(x, t)|) d x \leq e^{-d_{5} t} \int_{U} & H(|\nabla p(x, 0)|) d x \\
& +C \int_{0}^{t} e^{-d_{5}(t-\tau)}\left[\int_{U} \bar{p}^{2}(x, \tau) d x+G_{3}(\tau)\right] d \tau .
\end{array}
$$

Using estimate (4.13) in (4.28) gives

$$
\begin{aligned}
\int_{U} H(|\nabla p(x, t)|) d x & \leq e^{-d_{5} t} \int_{U} H(|\nabla p(x, 0)|) d x \\
& +C \int_{0}^{t} e^{-d_{5}(t-\tau)}\left(1+\int_{U}|p(0, x)|^{\widehat{2}} d x+[\operatorname{Env} A(\widehat{2}, \tau)]^{\frac{\widehat{2}}{2-a}}+G_{3}(\tau)\right) d \tau,
\end{aligned}
$$

and hence (4.22) follows.

(ii) Assume $A(\widehat{2})<\infty$. We notice that the first term on the right-hand side of (4.28) exponentially decays to 0 as $t \rightarrow \infty$. Next, by (2.35) and (4.14)

$$
\limsup _{t \rightarrow \infty} \int_{0}^{t} e^{-d_{5}(t-\tau)} \int_{U} \bar{p}(x, \tau)^{2} d x d \tau \leq C \limsup _{t \rightarrow \infty} \int_{U} \bar{p}^{2}(x, t) d x \leq C\left(1+A(\widehat{2})^{\gamma_{0}(\widehat{2})}\right) .
$$


Hence it follows from (4.28) for large $t$ that

$$
\int_{U} H(|\nabla p(x, t)|) d x \leq C\left(1+A(\widehat{2})^{\gamma_{0}(\widehat{2})}\right)+C \int_{U} e^{-d_{5}(t-\tau)} G_{3}(\tau) d \tau,
$$

which is (4.23). Taking limit superior both sides of (4.23) and, again, applying (2.35) to $\int_{0}^{t} e^{-d_{5}(t-\tau)} G_{3}(\tau) d \tau$ we obtain (4.24).

(iii) Assume $\beta(\widehat{2})<\infty$. By virtue of Theorem 4.3, there is $T_{1}>0$ such that (4.15) holds for $t>T_{1}$. Let $t>T_{1}$. Splitting the time integral in (4.28) gives

$$
\begin{aligned}
\int_{U} H(|\nabla p(x, t)|) d x \leq & e^{-d_{5} t} \int_{U} H(|\nabla p(x, 0)|) d x \\
& +C \int_{0}^{T_{1}} e^{-d_{5}(t-\tau)}\left(\int_{U} \bar{p}(x, \tau)^{2} d x+G_{3}(\tau)\right) d \tau \\
& +C \int_{T_{1}}^{t} e^{-d_{5}(t-\tau)}\left(\int_{U} \bar{p}(x, \tau)^{2} d x+G_{3}(\tau)\right) d \tau
\end{aligned}
$$

To estimate $\int_{U} \bar{p}(x, \tau)^{2} d x$ we use (4.13) for $\tau<T_{1}$ and use (4.15) for $t>T_{1}$. Then

$$
\begin{aligned}
& \int_{U} H(|\nabla p(x, t)|) d x \leq e^{-d_{5} t} \int_{U} H(|\nabla p(x, 0)|) d x \\
& \quad+C e^{-d_{5} t} \int_{0}^{T_{1}} e^{d_{5} \tau}\left(1+B(\widehat{2}, \tau)+G_{3}(\tau)\right) d \tau \\
& \quad+C \int_{T_{1}}^{\infty} e^{-d_{5}(t-\tau)}\left(1+\beta(\widehat{2})^{\frac{\widehat{2}}{2-2 a}}+A(\widehat{2}, \tau)^{\gamma_{0}(\widehat{2})}+G_{3}(\tau)\right) d \tau . \\
& \leq C e^{-d_{5} t}\left\{\int_{U} H(|\nabla p(x, 0)|) d x+\int_{0}^{T_{1}} e^{d_{5} \tau}\left(1+B(\widehat{2}, \tau)+G_{3}(\tau)\right) d \tau\right\} \\
& \quad+C\left(1+\beta(\widehat{2})^{\frac{\widehat{2}}{2-2 a}}\right)+C \int_{T_{1}}^{t} e^{-d_{5}(t-\tau)}\left(A(\widehat{2}, \tau)^{\gamma_{0}(\widehat{2})}+G_{3}(\tau)\right) d \tau .
\end{aligned}
$$

Due to the exponential decay of the term $e^{-d_{5} t}\{\ldots\}$ above, there exists $T>T_{1}$ such that for $t>T$,

$$
\int_{U} H(|\nabla p(x, t)|) d x \leq C\left(1+\beta(\widehat{2})^{\frac{\widehat{2}}{2-2 a}}\right)+C \int_{T_{1}}^{t} e^{-d_{5}(t-\tau)}\left(A(\widehat{2}, \tau)^{\gamma_{0}(\widehat{2})}+G_{3}(\tau)\right) d \tau,
$$

therefore we obtain (4.25).

For the time derivative, we have the following.

Theorem 4.5. (i) If $t \geq t_{0}>0$ then

$$
\begin{aligned}
& \int_{U} H(|\nabla p(x, t)|)+\left|\bar{p}_{t}(x, t)\right|^{2} d x \leq C\left(1+\int_{U}|\bar{p}(x, 0)|^{\widehat{2}} d x\right) \\
& +C e^{d_{6} t_{0}} t_{0}^{-1} e^{-d_{6} t}\left\{\int_{U}|\bar{p}(x, 0)|^{2}+H(|\nabla p(x, 0)|) d x+\int_{0}^{t_{0}} G_{3}(\tau) d \tau\right\} \\
& +C \int_{0}^{t} e^{-d_{6}(t-\tau)}\left([\operatorname{Env} A(\widehat{2}, \tau)]^{\frac{\widehat{2}}{2-a}}+G_{4}(\tau)\right) d \tau,
\end{aligned}
$$

where $d_{6}>0$ is introduced in (4.33) below. 
(ii) If $A(\widehat{2})<\infty$ then there is $T>0$ such that for $t>T$,

$$
\int_{U} H(|\nabla p(x, t)|)+\left|\bar{p}_{t}(x, t)\right|^{2} d x \leq C\left(1+A(\widehat{2})^{\frac{\widehat{2}}{2-a}}+\int_{1}^{t} e^{-d_{6}(t-\tau)} G_{4}(\tau) d \tau\right) ;
$$

consequently,

$$
\limsup _{t \rightarrow \infty} \int_{U} H(|\nabla p(x, t)|)+\left|\bar{p}_{t}(x, t)\right|^{2} d x \leq C\left[1+A(\widehat{2})^{\frac{\widehat{2}}{2-a}}+\limsup _{t \rightarrow \infty} G_{4}(t)\right] .
$$

(iii) If $\beta(\widehat{2})<\infty$ then there is $T>0$ such that for $t>T$,

$$
\int_{U} H(|\nabla p(x, t)|)+\left|\bar{p}_{t}(x, t)\right|^{2} d x \leq C\left(1+\beta(\widehat{2})^{\frac{\widehat{2}}{2-2 a}}+\int_{1}^{t} e^{-d_{6}(t-\tau)}\left(A(\widehat{2}, \tau)^{\frac{\widehat{2}}{2-a}}+G_{4}(\tau)\right) d \tau\right) .
$$

Proof. (i) The inequality (3.51) of [13] reads

$$
\frac{d}{d t} \int_{U} H(|\nabla p|)+\left|\bar{p}_{t}\right|^{2}+|\bar{p}|^{2} d x \leq-d_{6} \int_{U} H(|\nabla p|)+\left|\bar{p}_{t}\right|^{2} d x+C G_{4}(t) .
$$

By Cauchy's inequality (see the derivation of (4.26)), we have

$$
\frac{d}{d t} \int_{U} H(|\nabla p|)+\left|\bar{p}_{t}\right|^{2} d x \leq-d_{6} \int_{U} H(|\nabla p|)+\left|\bar{p}_{t}\right|^{2} d x+C \int_{U} \bar{p}^{2} d x+C G_{4}(t) \text {. }
$$

Then by Theorem 4.3(i),

$$
\frac{d}{d t} \int_{U} H(|\nabla p|)+\left|\bar{p}_{t}\right|^{2} d x \leq-d_{6} \int_{U} H(|\nabla p|)+\left|\bar{p}_{t}\right|^{2} d x+C(1+B(\widehat{2}, t))+C G_{4}(t) \text {. }
$$

By (4.19) and (4.20)

$$
\begin{aligned}
\int_{0}^{t_{0}} \int_{U} H(|\nabla p(x, \tau)|)+ & \left|\bar{p}_{t}(x, \tau)\right|^{2} d x d \tau \\
& \leq C_{1}\left[\int_{U} H(|\nabla p(x, 0)|)+|\bar{p}(x, 0)|^{2} d x+\int_{0}^{t_{0}} G_{3}(\tau) d \tau\right] .
\end{aligned}
$$

Hence there is $t^{\prime} \in\left(0, t_{0}\right)$ such that

$$
\begin{aligned}
\int_{U} H\left(\left|\nabla p\left(x, t^{\prime}\right)\right|\right)+ & \left|\bar{p}_{t}\left(x, t^{\prime}\right)\right|^{2} d x \\
& \leq 2 C_{1} t_{0}^{-1}\left[\int_{U} H(|\nabla p(x, 0)|)+|\bar{p}(x, 0)|^{2} d x+\int_{0}^{t_{0}} G_{3}(\tau) d \tau\right] .
\end{aligned}
$$

By (4.34) and Gronwall's inequality, for $t \geq t_{0}>t^{\prime}>0$,

$$
\begin{aligned}
& \int_{U} H(|\nabla p(x, t)|)+\left|\bar{p}_{t}(x, t)\right|^{2} d x \\
& \leq e^{-d_{6}\left(t-t^{\prime}\right)} \int_{U} H\left(\left|\nabla p\left(x, t^{\prime}\right)\right|\right)+\left|\bar{p}_{t}\left(x, t^{\prime}\right)\right|^{2} d x+C \int_{t^{\prime}}^{t} e^{-d_{6}(t-\tau)}\left(1+B(\widehat{2}, \tau)+G_{4}(\tau)\right) d \tau \\
& \leq C e^{-d_{6}\left(t-t_{0}\right)} t_{0}^{-1}\left\{\int_{U} H(|\nabla p(x, 0)|)+|\bar{p}(x, 0)|^{2} d x+\int_{0}^{t_{0}} G_{3}(\tau) d \tau\right\} \\
& \quad+C \int_{0}^{t} e^{-d_{6}(t-\tau)}\left(1+\int_{U} \bar{p}(x, 0)^{\widehat{2}} d x+[\operatorname{Env} A(\widehat{2}, \tau)]^{\gamma_{0}(\widehat{2})}+G_{4}(\tau)\right) d \tau .
\end{aligned}
$$


Therefore (4.29) follows.

(ii) Assume $A(\widehat{2})<\infty$. By (4.33) and Theorem 4.3(ii), there exists $T_{1} \geq 1$ such that

$$
\frac{d}{d t} \int_{U} H(|\nabla p|)+\left|\bar{p}_{t}\right|^{2} d x \leq-d_{6} \int_{U} H(|\nabla p|)+\left|\bar{p}_{t}\right|^{2} d x+C\left[1+A^{\gamma_{0}(\widehat{2})}+G_{4}(t)\right]
$$

for all $t>T_{1}$. Let $t>T_{1}$. Applying Gronwall's inequality to (4.35) yields

$$
\begin{aligned}
& \int_{U} H(|\nabla p(x, t)|)+\left|\bar{p}_{t}(x, t)\right|^{2} d x \\
& \leq e^{-d_{6}\left(t-T_{1}\right)} \int_{U} H\left(\left|\nabla p\left(x, T_{1}\right)\right|\right)+\bar{p}_{t}^{2}\left(x, T_{1}\right) d x+C \int_{T_{1}}^{t} e^{-d_{6}(t-\tau)}\left(1+A^{\gamma_{0}(\widehat{2})}+G_{4}(\tau)\right) d \tau \\
& \leq e^{-d_{6}\left(t-T_{1}\right)} \int_{U} H\left(\left|\nabla p\left(x, T_{1}\right)\right|\right)+\bar{p}_{t}^{2}\left(x, T_{1}\right) d x+C\left(1+A^{\gamma_{0}(\widehat{2})}\right)+C \int_{1}^{t} e^{-d_{6}(t-\tau)} G_{4}(\tau) d \tau .
\end{aligned}
$$

Since $\int_{U} H\left(\left|\nabla p\left(x, T_{1}\right)\right|\right)+\bar{p}_{t}^{2}\left(x, T_{1}\right) d x<\infty$, estimate (4.30) easily follows for sufficiently large $t$. Then (4.31) follows by taking limit superior of (4.30) and using (2.35).

(iii) Assume $\beta(\widehat{2})<\infty$. By Theorem 4.3 and (4.33) there is $T_{2}>0$ such that for $t>T_{2}$,

$$
\begin{aligned}
& \frac{d}{d t} \int_{U} H(|\nabla p|)+\left|\bar{p}_{t}\right|^{2} d x \\
& \quad \leq-d_{6} \int_{U} H(|\nabla p|)+\left|\bar{p}_{t}\right|^{2} d x+C\left[1+\beta(\widehat{2})^{\frac{\widehat{2}}{2-2 a}}+A(\widehat{2}, t)^{\gamma_{0}(\widehat{2})}+G_{4}(t)\right] .
\end{aligned}
$$

We then obtain (4.34) by using the same argument as in part (ii).

Remark 4.6. In the NDC case, despite the initial data being imposed to be in a higher Lebesgue space $L^{\alpha_{*}}(U)$ the estimates obtained in Theorems 4.4 and 4.5 are much sharper than those in [13]. In particularly if the boundary data satisfy $A(\widehat{2}, t)+G_{4}(t) \leq C$ for all $t \geq 0$, then $\int_{U} H(|\nabla p(x, t)|) d x$ is uniformly bounded on $[0, \infty)$, and $\int_{U}\left|\bar{p}_{t}(x, t)\right|^{2} d x$ is uniformly bounded on $[1, \infty)$. These cannot be achieved by the results in [13] which only yield the boundedness for finite time intervals.

We now estimate some integrals with mixed products of $p$ and $|\nabla p|$. These will be needed in section 6 .

Corollary 4.7. If $\alpha \geq \max \left\{2, \alpha_{*}\right\}$ and $t>0$ then

$$
\begin{array}{rl}
\int_{0}^{t} \int_{U}|\nabla p(x, \tau)|^{2-a}|\bar{p}(x, \tau)|^{\alpha-2} & d x d \tau \\
& \leq C\left(\int_{U}|\bar{p}(x, 0)|^{\alpha} d x+\int_{0}^{t}(1+A(\alpha, \tau)) d \tau\right),
\end{array}
$$




$$
\begin{aligned}
\int_{U}|\nabla p(x, t)|^{2-a} & |\bar{p}(x, t)|^{\alpha-2} d x \\
& \leq C\left(1+A(\alpha, t)+\int_{U}|\bar{p}(x, t)|^{2(\alpha-1)} d x+\int_{U}\left|\bar{p}_{t}(x, t)\right|^{2} d x\right) .
\end{aligned}
$$

Proof. Note in this case that $\widehat{\alpha}=\alpha$. Since $|\nabla p| \leq|\nabla \bar{p}|+|\nabla \Psi|$, we have

$$
\int_{U}|\nabla p|^{2-a}|\bar{p}|^{\alpha-2} d x \leq C \int_{U}|\nabla \bar{p}|^{2-a}|\bar{p}|^{\alpha-2} d x+C \int_{U}|\nabla \Psi|^{2-a}|\bar{p}|^{\alpha-2} d x .
$$

Let $t>0$. Integrating inequality (4.11) from 0 to $t$ with $\varepsilon=1$ gives

$$
\begin{aligned}
\int_{0}^{t} \int_{U}|\nabla \bar{p}|^{2-a}|\bar{p}|^{\alpha-2} d x & \leq C \int_{U}|\bar{p}(x, 0)|^{\alpha} d x-C \int_{U}|\bar{p}(x, t)|^{\alpha} d x \\
& +\int_{0}^{t}\left(\int_{U}|\bar{p}(x, \tau)|^{\alpha} d x\right)^{\frac{1}{\gamma_{0}}} d \tau+C \int_{0}^{t}(1+A(\alpha, \tau)) d \tau .
\end{aligned}
$$

Integrating (4.3) from 0 to $t$ yields

$$
\int_{0}^{t}\left(\int_{U}|\bar{p}(x, \tau)|^{\alpha} d x\right)^{\frac{1}{\gamma_{0}}} d \tau \leq C \int_{U}|\bar{p}(x, 0)|^{\alpha} d x+C \int_{0}^{t} 1+A(\alpha, \tau) d \tau .
$$

Combining (4.40) with (4.41) we have

$$
\int_{0}^{t} \int_{U}|\nabla \bar{p}|^{2-a}|\bar{p}|^{\alpha-2} d x \leq C \int_{U}|\bar{p}(x, 0)|^{\alpha} d x+C \int_{0}^{t}(1+A(\alpha, \tau)) d \tau .
$$

By (4.39) as well as Hölder's and Young's inequalities,

$$
\begin{aligned}
& \int_{0}^{t} \int_{U}|\nabla p|^{2-a}|\bar{p}|^{\alpha-2} d x d \tau \\
& \leq C \int_{0}^{t} \int_{U}|\nabla \bar{p}|^{2-a}|\bar{p}|^{\alpha-2} d x d \tau+C \int_{0}^{t} \int_{U}|\nabla \Psi|^{2-a}|\bar{p}|^{\alpha-2} d x d \tau \\
& \leq C \int_{0}^{t} \int_{U}|\nabla \bar{p}|^{2-a}|\bar{p}|^{\alpha-2} d x d \tau+C \int_{0}^{t}\left(\int_{U}|\nabla \Psi|^{\frac{\alpha(2-a)}{2}} d x\right)^{\frac{2}{\alpha}}\left(\int_{U}|\bar{p}|^{\alpha} d x\right)^{\frac{\alpha-2}{\alpha}} d \tau \\
& \leq C \int_{0}^{t} \int_{U}|\nabla \bar{p}|^{2-a}|\bar{p}|^{\alpha-2} d x d \tau+C \int_{0}^{t}\left(\int_{U}|\nabla \Psi|^{\frac{\alpha(2-a)}{2}} d x\right)^{\frac{2(\alpha-a)}{\alpha(2-a)}} d \tau \\
& \quad+\int_{0}^{t}\left(\int_{U}|\bar{p}|^{\alpha} d x\right)^{\frac{1}{\gamma_{0}}} d \tau .
\end{aligned}
$$

Utilizing estimates (4.41) and (4.42) in the last inequality, and noticing that the quantity $\left(\int_{U}|\nabla \Psi(x, \tau)|^{\frac{\alpha(2-a)}{2}} d x\right)^{\frac{2(\alpha-a)}{\alpha(2-a)}}$ is a part of $A(\alpha, \tau)$ we obtain (4.37).

Now, we set $\varepsilon=1$ in (4.11) and rewrite it as

$$
\int_{U}|\nabla \bar{p}|^{2-a}|\bar{p}|^{\alpha-2} d x \leq-C \int_{U} \frac{\partial|\bar{p}|^{\alpha}}{\partial t} d x+C \int_{U}|\bar{p}|^{\alpha} d x+C(1+A(\alpha, t)) .
$$

Hence

$$
\begin{aligned}
\int_{U}|\nabla \bar{p}|^{2-a}|\bar{p}|^{\alpha-2} d x & \leq C \int_{U}\left|\bar{p}_{t}\right||\bar{p}|^{\alpha-1} d x+C \int_{U}|\bar{p}|^{\alpha} d x+C(1+A(\alpha, t)) \\
& \leq C\left\{\int_{U}\left|\bar{p}_{t}\right|^{2} d x+\int_{U}|\bar{p}|^{2(\alpha-1)} d x+\int_{U}|\bar{p}|^{\alpha} d x+1+A(\alpha, t)\right\} .
\end{aligned}
$$


Also, by Young's inequality

$$
\int_{U}|\nabla \Psi|^{2-a}|\bar{p}|^{\alpha-2} d x \leq \int_{U}|\nabla \Psi|^{\frac{\alpha(2-a)}{2}} d x+\int_{U}|\bar{p}|^{\alpha} d x \leq 1+A(\alpha, t)+\int_{U}|\bar{p}|^{\alpha} d x .
$$

The last inequality is due to the fact that $\frac{2(\alpha-1)}{\alpha(2-a)} \geq 1$. Thus we have from (4.39),

$$
\begin{aligned}
\int_{U}|\nabla p|^{2-a}|\bar{p}|^{\alpha-2} d x \leq & C\left\{\int_{U}\left|\bar{p}_{t}\right|^{2} d x+\int_{U}|\bar{p}|^{2(\alpha-1)} d x+\int_{U}|\bar{p}|^{\alpha} d x+1+A(\alpha, t)\right\} \\
& +C\left\{1+A(\alpha, t)+\int_{U}|\bar{p}|^{\alpha} d x\right\} .
\end{aligned}
$$

Since $2(\alpha-1) \geq \alpha$, estimate (4.38) then follows.

Remark 4.8. The estimates and corresponding conditions in Theorems 4.3, 4.4 and 4.5 need be made more flexible in order to compare one to another when the involved parameters are varied. This can be done in the following way. First consider Theorem 4.3. Suppose

$$
\alpha^{\prime} \geq \widehat{\alpha} \text { and } A\left(\alpha^{\prime}, t\right)^{\gamma_{0}\left(\alpha^{\prime}\right)} \leq C_{\alpha^{\prime}}(1+G(t)),
$$

where $C_{\alpha^{\prime}}$ denotes a generic positive constant depending on $\alpha^{\prime}$. Then we can replace constant $C$ depending on $\alpha$ by a constant $C_{\alpha^{\prime}}$ depending on $\alpha^{\prime}$; and

- in part (i), replace $\int_{U}|\bar{p}(x, 0)|^{\widehat{\alpha}} d x$ by $\int_{U}|\bar{p}(x, 0)|^{\alpha^{\prime}} d x$, replace $[E n v A(\widehat{\alpha}, \cdot)]^{\frac{\widehat{\alpha}}{\alpha-a}}$ by $\operatorname{Env} G(\cdot)$; and

- in part (ii), replace $A(\widehat{\alpha})$ by $\left[\lim \sup _{t \rightarrow \infty} G(t)\right]^{\frac{1}{\gamma_{0}\left(\alpha^{\prime}\right)}}$, replace $A(\widehat{\alpha})^{\frac{\widehat{\alpha}}{\alpha-a}}$ by $\lim \sup _{t \rightarrow \infty} G(t)$; and

- in part (iii), replace $\beta(\widehat{\alpha})$ by $\beta^{\prime}=\limsup _{t \rightarrow \infty}\left[\left(G(t)^{\frac{1}{\gamma_{0}\left(\alpha^{\prime}\right)}}\right)^{\prime}\right]^{-}$, replace $\beta(\widehat{\alpha})^{\frac{\widehat{\alpha}}{\widehat{\alpha}-2 a}}$ by $\beta^{\prime \frac{\alpha^{\prime}}{\alpha^{\prime}-2 a}}$, replace $A(\widehat{\alpha}, t)^{\frac{\widehat{\alpha}}{\alpha-a}}$ by $G(t)$.

Indeed, by Young's inequality

$$
\int_{U}|\bar{p}(x, t)|^{\alpha} d x \leq \int_{U} 1+|\bar{p}(x, t)|^{\alpha^{\prime}} d x
$$

and by (4.3) and (4.43),

$$
\frac{d}{d t} \int_{U}|\bar{p}(x, t)|^{\alpha^{\prime}} d x \leq-c_{5}\left(\alpha^{\prime}\right)\left(\int_{U}|\bar{p}(x, t)|^{\alpha^{\prime}} d x\right)^{\frac{1}{\gamma_{0}\left(\alpha^{\prime}\right)}}+C_{\alpha^{\prime}}\left(1+G(t)^{\frac{1}{\gamma_{0}\left(\alpha^{\prime}\right)}}\right), \quad t>0 .
$$

Then we can proceed the proof of Theorem 4.3 with $\alpha^{\prime}$ replacing $\alpha$ and $G(t)^{\frac{1}{\gamma_{0}\left(\alpha^{\prime}\right)}}$ replacing $A(\alpha, t)$, also noticing that $\widehat{\alpha^{\prime}}=\alpha^{\prime}$,

$$
\begin{gathered}
\limsup _{t \rightarrow \infty}\left[G(t)^{\frac{1}{\gamma_{0}\left(\alpha^{\prime}\right)}}\right]=\left[\limsup _{t \rightarrow \infty} G(t)\right]^{\frac{1}{\gamma_{0}\left(\alpha^{\prime}\right)}}, \\
\left\{\limsup _{t \rightarrow \infty}\left[G(t)^{\frac{1}{\gamma_{0}\left(\alpha^{\prime}\right)}}\right]\right\}^{\gamma_{0}\left(\alpha^{\prime}\right)}=\limsup _{t \rightarrow \infty} G(t),
\end{gathered}
$$

and we can choose $[\operatorname{Env} G(t)]^{\frac{1}{\gamma_{0}\left(\alpha^{\prime}\right)}}$ for $\operatorname{Env}\left[G(t)^{\frac{1}{\gamma_{0}\left(\alpha^{\prime}\right)}}\right]$, therefore

$$
\left\{\operatorname{Env}\left[G(t)^{\frac{1}{\gamma_{0}\left(\alpha^{\prime}\right)}}\right]\right\}^{\gamma_{0}\left(\alpha^{\prime}\right)}=\operatorname{Env} G(t) .
$$

The above replacements can also be applied to Theorems 4.4 and 4.5 with $\widehat{\alpha}=\widehat{2}$. 


\section{DEPENDENCE ON THE BOUNDARY DATA}

In this section we study the continuous dependence of the solution to IBVP (3.1) on the boundary data $\psi(x, t)$. Let $p_{k}=p_{k}(x, t)$, for $k=1,2$, be two solutions of (3.1) with boundary values $\psi_{k}$ respectively. For $k=1,2$, let $\Psi_{k}$ be an extension of $\psi_{k}$ and denote $\bar{p}_{k}=p_{k}-\Psi_{k}$. Let

$$
z=p_{1}-p_{2}, \Phi=\Psi_{1}-\Psi_{2} \text { and } \bar{z}=\bar{p}_{1}-\bar{p}_{2}=z-\Phi .
$$

We will estimate $\int_{U}|\bar{z}(x, t)|^{\alpha} d x$ and $\int_{U}|\nabla \bar{z}(x, t)|^{2-a} d x$. The corresponding estimates for $z$ can be retrieved easily by using the relations

$$
\begin{gathered}
\int_{U}|z|^{\alpha} d x \leq C\left[\int_{U}|\bar{z}|^{\alpha} d x+\int_{U}|\Phi|^{\alpha} d x\right], \\
\int_{U}|\nabla z|^{2-a} d x \leq C\left[\int_{U}|\nabla \bar{z}|^{2-a} d x+\int_{U}|\nabla \Phi|^{2-a} d x\right] .
\end{gathered}
$$

We derive from (5.1) then IBVP for $\bar{z}(x, t)$ :

$$
\left\{\begin{array}{l}
\frac{\partial \bar{z}}{\partial t}=\nabla \cdot\left(K\left(\left|\nabla p_{1}\right|\right) \nabla p_{1}-K\left(\left|\nabla p_{2}\right|\right) \nabla p_{2}\right)-\Phi_{t} \text { in } U \times(0, \infty), \\
\bar{z}(x, 0)=\bar{p}_{1}(x, 0)-\bar{p}_{2}(x, 0) \text { in } U, \\
\bar{z}(x, t)=0 \text { on } \Gamma \times(0, \infty) .
\end{array}\right.
$$

Throughout this section the numbers $\theta, \theta_{1}$ and $\theta_{2}$ are as in Lemma 2.5. Denote

$$
N(\lambda, t)=\sum_{k=1}^{2}\left(\int_{U}\left|\bar{p}_{k}(x, t)\right|^{\lambda} d x\right)^{1 / \lambda} \text { for } \lambda>0, \text { and } N(0, t)=1 .
$$

Lemma 5.1. Let $\alpha \geq 2$.

(i) In the DC case,

$$
\frac{d}{d t} \int_{U}|\bar{z}(x, t)|^{\alpha} d x \leq-c_{6}\left[\int_{U}|\bar{z}(x, t)|^{\alpha} d x\right] M_{1}(t)^{-\frac{a}{2-a}}+C F(\alpha, t) D(\alpha, t)
$$

for all $t>0$, where $c_{6}=c_{6}(\alpha, \vec{\alpha}, \vec{a}, n, U)>0$,

$$
\begin{aligned}
& M_{1}(t)=1+\int_{U}\left|\nabla p_{1}(x, t)\right|^{2-a}+\left|\nabla p_{2}(x, t)\right|^{2-a} d x, \\
& F(\alpha, t)=1+N(\alpha, t)^{\alpha-1}+N\left(\gamma_{1}, t\right)^{\alpha-2} M_{1}(t)^{\frac{1-a}{2-a}},
\end{aligned}
$$

with $\gamma_{1}=\gamma_{1}(\alpha) \stackrel{\text { def }}{=} 2(\alpha-2)(2-a)$, and

$$
D(\alpha, t)= \begin{cases}\|\nabla \Phi(\cdot, t)\|_{L^{2(2-a)}}+\|\nabla \Phi(\cdot, t)\|_{L^{\alpha}}^{2}+\left\|\Phi_{t}(\cdot, t)\right\|_{L^{\alpha}} & \text { if } \alpha>2 \\ \|\nabla \Phi(\cdot, t)\|_{L^{2-a}}+\|\nabla \Phi(\cdot, t)\|_{L^{2}}^{2}+\left\|\Phi_{t}(\cdot, t)\right\|_{L^{2}} & \text { if } \alpha=2 .\end{cases}
$$

(ii) In the NDC case,

$$
\frac{d}{d t} \int_{U}|\bar{z}(x, t)|^{\alpha} d x \leq-c_{7}\left[\int_{U}|\bar{z}(x, t)|^{\alpha} d x\right]^{\theta} M_{2}(\alpha, t)^{-\frac{2-\theta_{1}}{\theta_{1}}}+C F(\alpha, t) D(\alpha, t)
$$

for all $t>0$, where $c_{7}=c_{7}\left(\alpha, \theta, \theta_{1}, \vec{\alpha}, \vec{a}, n, U\right)>0$,

$$
M_{2}(\alpha, t)=1+\int_{U}\left|\nabla p_{1}(x, t)\right|^{2-a}+\left|\nabla p_{2}(x, t)\right|^{2-a}+\left|\bar{p}_{1}(x, t)\right|^{\theta_{2} \alpha}+\left|\bar{p}_{2}(x, t)\right|^{\theta_{2} \alpha} d x \text {. }
$$


Proof. Multiplying the first equation of (5.1) by $|\bar{z}|^{\alpha-1} \operatorname{sign}(z)$, integrating over domain $U$, then applying Green's formula, we have

$$
\begin{aligned}
& \frac{1}{\alpha} \frac{d}{d t} \int_{U}|\bar{z}|^{\alpha} d x \\
& =-(\alpha-1) \int_{U}\left(K\left(\left|\nabla p_{1}\right|\right) \nabla p_{1}-K\left(\left|\nabla p_{2}\right|\right) \nabla p_{2}\right) \cdot \nabla \bar{z}|\bar{z}|^{\alpha-2} d x-\int_{U} \Phi_{t}|\bar{z}|^{\alpha-1} \operatorname{sign}(\bar{z}) d x \\
& =-(\alpha-1) \int_{U}\left(K\left(\left|\nabla p_{1}\right|\right) \nabla p_{1}-K\left(\left|\nabla p_{2}\right|\right) \nabla p_{2}\right) \cdot\left(\nabla p_{1}-\nabla p_{2}\right)|\bar{z}|^{\alpha-2} d x \\
& \quad+(\alpha-1) \int_{U}\left(K\left(\left|\nabla p_{1}\right|\right) \nabla p_{1}-K\left(\left|\nabla p_{2}\right|\right) \nabla p_{2}\right) \cdot \nabla \Phi|\bar{z}|^{\alpha-2} d x-\int_{U} \Phi_{t}|\bar{z}|^{\alpha-1} \operatorname{sign}(\bar{z}) d x .
\end{aligned}
$$

By the monotonicity, c.f. Lemma 2.1(i),

$$
\begin{aligned}
\frac{1}{\alpha} \frac{d}{d t} \int_{U}|\bar{z}|^{\alpha} d x \leq & -(\alpha-1)(1-a) \int_{U} K\left(\max \left\{\left|\nabla p_{1}\right|,\left|\nabla p_{2}\right|\right\}\right)\left|\nabla p_{1}-\nabla p_{2}\right|^{2}|\bar{z}|^{\alpha-2} d x \\
& +(\alpha-1) J_{2}+J_{1}
\end{aligned}
$$

where

$$
\begin{aligned}
& J_{1}=\int_{U}\left|\Phi_{t}\right||\bar{z}|^{\alpha-1} d x \\
& J_{2}=\int_{U}\left(K\left(\left|\nabla p_{1}\right|\right)\left|\nabla p_{1}\right|+K\left(\left|\nabla p_{2}\right|\right)\left|\nabla p_{2}\right|\right)|\nabla \Phi \| \bar{z}|^{\alpha-2} d x .
\end{aligned}
$$

Since $\left|\nabla p_{1}-\nabla p_{2}\right|^{2}=|\nabla \bar{z}-\nabla \Phi|^{2} \geq 1 / 2|\nabla \bar{z}|^{2}-|\nabla \Phi|^{2}$, we have

$$
\frac{1}{\alpha} \frac{d}{d t} \int_{U}|\bar{z}|^{\alpha} d x \leq-C J_{4}+C J_{3}+(\alpha-1) J_{2}+J_{1}
$$

where

$$
\begin{aligned}
& J_{3}=\int_{U} K\left(\max \left\{\left|\nabla p_{1}\right|,\left|\nabla p_{2}\right|\right\}\right)|\nabla \Phi|^{2}|\bar{z}|^{\alpha-2} d x \\
& J_{4}=\int_{U} K\left(\max \left\{\left|\nabla p_{1}\right|,\left|\nabla p_{2}\right|\right\}\right)|\nabla \bar{z}|^{2}|\bar{z}|^{\alpha-2} d x .
\end{aligned}
$$

We now estimate each of $J_{1}, J_{2}, J_{3}$.

Consider the case $\alpha>2$. For $J_{1}$, we have

$$
J_{1} \leq C\left[\int_{U}\left|\Phi_{t}\right|^{\alpha} d x\right]^{\frac{1}{\alpha}}\left[\int_{U}|\bar{z}|^{\alpha} d x\right]^{\frac{\alpha-1}{\alpha}} \leq C\left\|\Phi_{t}\right\|_{L^{\alpha}} N(\alpha, t)^{\alpha-1} .
$$

For $J_{2}$, using (2.10) and applying Hölder's inequality give

$$
\begin{aligned}
J_{2} & \leq C \int_{U}\left(\sum_{k=1}^{2}\left|\nabla p_{k}\right|^{1-a}\right)|\nabla \Phi||\bar{z}|^{\alpha-2} d x \\
& \leq C\left[\sum_{k=1}^{2} \int_{U}\left|\nabla p_{k}\right|^{2-a} d x\right]^{\frac{1-a}{2-a}}\left[\int_{U}|\bar{z}|^{(\alpha-2)(2-a)}|\nabla \Phi|^{2-a} d x\right]^{\frac{1}{2-a}} \\
& \leq C M_{1}(t)^{\frac{1-a}{2-a}}\left[\int_{U}|\bar{z}|^{2(\alpha-2)(2-a)} d x\right]^{\frac{1}{2(2-a)}}\left[\int_{U}|\nabla \Phi|^{2(2-a)} d x\right]^{\frac{1}{2(2-a)}} .
\end{aligned}
$$


Hence

$$
J_{2} \leq C M_{1}(t)^{\frac{1-a}{2-a}} N(2(\alpha-2)(2-a), t)^{\alpha-2}\|\nabla \Phi\|_{L^{2(2-a)}} .
$$

For $J_{3}$, by the boundedness of $K(\xi)$, Hölder's and Young's inequalities, we get

$J_{3} \leq C \int_{U}|\nabla \Phi|^{2}|\bar{z}|^{\alpha-2} d x \leq C\left[\int_{U}|\nabla \Phi|^{\alpha} d x\right]^{\frac{2}{\alpha}}\left[\int_{U}|\bar{z}|^{\alpha} d x\right]^{\frac{\alpha-2}{\alpha}} \leq C\|\nabla \Phi\|_{L^{\alpha}}^{2} N(\alpha, t)^{\alpha-2}$,

thus,

$$
J_{3} \leq C\left(1+N(\alpha, t)^{\alpha-1}\right)\|\nabla \Phi\|_{L^{\alpha}}^{2} .
$$

Combining (5.8) with estimates (5.9), (5.10) and (5.11), we obtain

$$
\begin{aligned}
\frac{d}{d t} \int_{U}|\bar{z}|^{\alpha} d x \leq & -C J_{4}+C N(\alpha, t)^{\alpha-1}\left\|\Phi_{t}\right\|_{L^{\alpha}}+C M_{1}(t)^{\frac{1-a}{2-a}} N\left(\gamma_{1}, t\right)^{\alpha-2}\|\nabla \Phi\|_{L^{2(2-a)}} \\
& +C\left(1+N(\alpha, t)^{\alpha-1}\right)\|\nabla \Phi\|_{L^{\alpha}}^{2},
\end{aligned}
$$

hence

$$
\frac{d}{d t} \int_{U}|\bar{z}|^{\alpha} d x \leq-C J_{4}+C F(\alpha, t) D(\alpha, t) .
$$

For the case $\alpha=2$ we use the same estimate for $J_{1}$ while quickly estimate

$$
J_{2} \leq C M_{1}(t)^{\frac{1-a}{2-a}}\|\nabla \Phi\|_{L^{2-a}} \text { and } J_{3} \leq C\|\nabla \Phi\|_{L^{2}}^{2},
$$

hence obtaining (5.12) again with the corresponding $D(2, t)$ defined in (5.5).

(i) Consider the DC case. Applying inequality (2.25) of Lemma 2.5 with $u=\bar{z}$, $\xi=\max \left\{\left|\nabla p_{1}\right|,\left|\nabla p_{2}\right|\right\}$ and using $\xi \leq\left|\nabla p_{1}\right|+\left|\nabla p_{2}\right|$ in the last integral of that inequality we obtain

which implies

$$
\int_{U}|\bar{z}|^{\alpha} d x \leq C J_{4} M_{1}(t)^{\frac{a}{2-a}}
$$

$$
-J_{4} \leq-C\left[\int_{U}|\bar{z}|^{\alpha} d x\right] M_{1}(t)^{-\frac{a}{2-a}} .
$$

Then (5.2) follows from (5.12) and (5.13).

(ii) Now consider the NDC case. Similarly, applying inequality (2.27) instead of (2.25) with the same $u(x)$ and $\xi(x)$, and also using $|u| \leq\left|\bar{p}_{1}\right|+\left|\bar{p}_{2}\right|$ in its last integral, we have

$$
-J_{4} \leq-C\left[\int_{U}|\bar{z}|^{\alpha} d x\right]^{\theta} M_{2}(\alpha, t)^{-\frac{2-\theta_{1}}{\theta_{1}}}
$$

Hence (5.6) follows from (5.12) and (5.14).

Referring to the notation used in the section 4 , we set

$$
\mathcal{G}_{j}(t)=G_{j}\left[\Psi_{1}\right](t)+G_{j}\left[\Psi_{2}\right](t) \text { for } j=1,2,3,4 .
$$

To unify may estimates below, we will use the replacements in Remark 4.8. With the condition (4.43) in mind, we observe that

$$
\left(\sum_{k=1}^{2} A\left[\Psi_{k}\right](\alpha, t)\right)^{\gamma_{0}(\alpha)} \leq C \sum_{k=1}^{2}\left\{\left(\int_{U}\left|\nabla \Psi_{k}\right|^{\frac{\alpha(2-a)}{2}} d x\right)^{\frac{2}{2-a}}+\left(\int_{U}\left|\left(\Psi_{k}\right)_{t}\right|^{\alpha} d x\right)^{\frac{1}{1-a}}\right\},
$$


therefore, by Hölder's inequality,

$$
\left(\sum_{k=1}^{2} A\left[\Psi_{k}\right](\alpha, t)\right)^{\gamma_{0}(\alpha)} \leq C \tilde{\mathcal{A}}(\alpha, t)
$$

where

$$
\tilde{\mathcal{A}}(\alpha, t)=\sum_{k=1}^{2}\left\{\int_{U}\left|\nabla \Psi_{k}(x, t)\right|^{\alpha} d x+\left(\int_{U}\left|\left(\Psi_{k}(x, t)\right)_{t}\right|^{\alpha} d x\right)^{\frac{1}{1-a}}\right\} .
$$

For each $k=1,2$, applying (4.22) of Theorem 4.4 for $p_{k}$ and using replacements in Remark 4.8 with $\alpha^{\prime}=\alpha \geq \widehat{2}$ and, thanks to (5.15), $G(t)=\tilde{\mathcal{A}}(\alpha, t)$, we have

$$
M_{1}(t) \leq C_{\alpha} \bar{M}_{1}(\alpha, t) \text { for all } \alpha \geq \widehat{2}, t \geq 0 \text {, }
$$

where

$$
\begin{aligned}
\bar{M}_{1}(\alpha, t)=1+\sum_{k=1}^{2}\left[\int_{U}\left|\bar{p}_{k}(x, 0)\right|^{\alpha} d x\right. & \left.+e^{-d_{5} t} \int_{U}\left|\nabla p_{k}(x, 0)\right|^{2-a} d x\right] \\
& +\int_{0}^{t} e^{-d_{5}(t-\tau)}\left(\operatorname{Env} \tilde{\mathcal{A}}(\alpha, \tau)+\mathcal{G}_{3}(\tau)\right) d \tau .
\end{aligned}
$$

Particularly, for $\alpha=\widehat{2}$

$$
M_{1}(t) \leq C \bar{M}_{1}(t) \text { for all } t \geq 0, \text { where } \bar{M}_{1}(t)=\bar{M}_{1}(\widehat{2}, t) .
$$

For $\lambda \geq 0$, similar to (5.17) but using (4.13) instead of (4.22), using $\alpha^{\prime}=\lambda$ and $G(t)=\tilde{\mathcal{A}}(\lambda, t)$ in Remark 4.8, we have

$$
\sum_{k=1}^{2} \int_{U}\left|\bar{p}_{k}(\cdot, t)\right|^{\lambda} d x \leq C_{\lambda} \bar{E}(\widehat{\lambda}, t) \text { for all } t \geq 0
$$

where

$$
\bar{E}(\lambda, t)= \begin{cases}1+\sum_{k=1}^{2} \int_{U}\left|\bar{p}_{k}(\cdot, 0)\right|^{\lambda} d x+\operatorname{Env} \tilde{\mathcal{A}}(\lambda, t) & \text { if } \lambda>0 \\ 1 & \text { if } \lambda=0\end{cases}
$$

Let $\lambda \geq \widehat{\theta_{2} \alpha} \geq \widehat{2}$, we observe that

$$
\begin{aligned}
M_{2}(\alpha, t) & =M_{1}(t)+\sum_{k=1}^{2} \int_{U}\left|\bar{p}_{k}(x, t)\right|^{\theta_{2} \alpha} d x \\
& \leq C_{\alpha, \lambda}\left(1+M_{1}(t)+\sum_{k=1}^{2} \int_{U}\left|\bar{p}_{k}(x, t)\right|^{\lambda} d x\right) .
\end{aligned}
$$

hence by taking $\alpha$ in (5.17) to be $\lambda$ and using (5.20), we obtain

$$
M_{2}(\alpha, t) \leq C_{\alpha, \lambda}\left(\bar{M}_{1}(\lambda, t)+\bar{E}(\lambda, t)\right) .
$$

Taking into account that $\operatorname{Env} \tilde{A}(\lambda, t)$ is increasing in $t$, we can bound

$$
\int_{0}^{t} e^{-d_{5}(t-\tau)} \operatorname{Env} \tilde{\mathcal{A}}(\lambda, \tau) d \tau \leq d_{5}^{-1} \operatorname{Env} \tilde{A}(\lambda, t) .
$$


Hence

$$
M_{2}(\alpha, t) \leq C_{\alpha, \lambda} \bar{M}_{2}(\lambda, t) \text { for all } \lambda \geq \widehat{\theta_{2} \alpha}, t \geq 0
$$

where

$$
\begin{aligned}
\bar{M}_{2}(\lambda, t)=1+\sum_{k=1}^{2}\left[\int_{U}\left|\bar{p}_{k}(x, 0)\right|^{\lambda} d x\right. & \left.+e^{-d_{5} t} \int_{U}\left|\nabla p_{k}(x, 0)\right|^{2-a} d x\right] \\
& +\operatorname{Env} \tilde{\mathcal{A}}(\lambda, t)+\int_{0}^{t} e^{-d_{5}(t-\tau)} \mathcal{G}_{3}(\tau) d \tau .
\end{aligned}
$$

When $\lambda=\widehat{\theta_{2} \alpha}$, estimate $(5.25)$ reads

$$
M_{2}(\alpha, t) \leq C_{\alpha} \bar{M}_{2}\left(\widehat{\theta_{2} \alpha}, t\right) \text { for all } t \geq 0
$$

If $\alpha \geq 0$, then $N(\alpha, t) \leq C_{\alpha}(1+N(\widehat{\alpha}, t))$, and by (5.20) we have $N(\alpha, t) \leq$ $\left.C_{\alpha} \bar{E}(\widehat{\alpha}, t)\right)^{1 / \widehat{\alpha}}$, thus

$$
N(\alpha, t) \leq C_{\alpha} \bar{N}(\alpha, t) \text { for all } \geq \alpha \geq 0, t \geq 0,
$$

where

$$
\bar{N}(\alpha, t)= \begin{cases}1+\sum_{k=1}^{2}\left\|\bar{p}_{k}(\cdot, 0)\right\|_{L^{\widehat{\alpha}}}+(\operatorname{Env} \tilde{\mathcal{A}}(\widehat{\alpha}, t))^{1 / \widehat{\alpha}} & \text { if } \alpha>0 \\ 1 & \text { if } \alpha=0 .\end{cases}
$$

If $\lambda \geq \alpha \geq 0$, then $N(\alpha, t) \leq C_{\alpha, \lambda}(1+N(\lambda, t))$, and applying (5.28) with $\alpha$ set to equal $\lambda$, we have

$$
N(\alpha, t) \leq C_{\alpha, \lambda} \bar{N}(\lambda, t) \text { for all } \lambda \geq \alpha \geq 0, t \geq 0 .
$$

Consequently, concerning $F(\alpha, t)$ in (5.4), using (5.28) to estimate $N(\alpha, t)$ and $N\left(\gamma_{1}, t\right)$, and using (5.19) to estimate $M_{1}(t)$, we obtain

$$
F(\alpha, t) \leq C_{\alpha} \bar{F}(\alpha, t) \text { for all } t \geq 0
$$

where

$$
\bar{F}(\alpha, t)=\bar{N}(\alpha, t)^{\alpha-1}+\bar{N}\left(\gamma_{1}(\alpha), t\right)^{\alpha-2} \bar{M}_{1}(t)^{\frac{1-a}{2-a}} .
$$

Alternatively, for $\lambda \geq \max \left\{\alpha, \gamma_{1}(\alpha), \widehat{\theta_{2} \alpha}\right\}$, we use (5.30) to estimate $N(\alpha, t)$ and $N\left(\gamma_{1}, t\right)$, and use (5.17) with $\alpha$ set to equal $\lambda$ to estimate $M_{1}(t)$, thus obtaining

$$
F(\alpha, t) \leq C_{\alpha, \lambda} \bar{F}(\alpha, \lambda, t) \text { for all } t \geq 0, \lambda \geq \max \left\{\alpha, \gamma_{1}(\alpha), \widehat{\theta_{2} \alpha}\right\}
$$

where

$$
\bar{F}(\alpha, \lambda, t)=\bar{N}(\lambda, t)^{\alpha-1}+\bar{N}(\lambda, t)^{\alpha-2} \bar{M}_{1}(\lambda, t)^{\frac{1-a}{2-a}} .
$$

For large time estimates we define

$$
\bar{A}(\alpha)=\limsup _{t \rightarrow \infty} \tilde{\mathcal{A}}(\alpha, t) \text { and } \bar{\beta}(\alpha)=\limsup _{t \rightarrow \infty}\left[\frac{d}{d t}\left(\tilde{\mathcal{A}}(\alpha, t)^{\frac{1}{\gamma_{0}(\alpha)}}\right)\right]^{-} .
$$

For $\alpha \geq \widehat{2}$, there is $T_{\alpha}>0$ such that

$$
M_{1}(t) \leq C_{\alpha} \tilde{M}_{1}(\alpha, t) \text { for all } t \geq T_{\alpha},
$$


where

$$
\tilde{M}_{1}(\alpha, t)= \begin{cases}1+\bar{A}(\alpha)+\int_{0}^{t} e^{-d_{5}(t-\tau)} \mathcal{G}_{3}(\tau) d \tau & \text { if } \bar{A}(\alpha)<\infty \\ 1+\bar{\beta}(\alpha)^{\frac{\alpha}{\alpha-2 a}} & \\ +\int_{0}^{t} e^{-d_{5}(t-\tau)}\left(\tilde{\mathcal{A}}(\alpha, \tau)+\mathcal{G}_{3}(\tau)\right) d \tau & \text { if } \bar{A}(\alpha)=\infty, \bar{\beta}(\alpha)<\infty \\ 1+\operatorname{Env} \tilde{\mathcal{A}}(\alpha, t) & \text { if } \bar{A}(\alpha)=\infty, \bar{\beta}(\alpha)=\infty \\ +\int_{0}^{t} e^{-d_{5}(t-\tau)} \mathcal{G}_{3}(\tau) d \tau & \end{cases}
$$

In particular, when $\alpha=\widehat{2}$ there is $T>0$ such that

$$
M_{1}(t) \leq C \tilde{M}_{1}(t) \text { for all } t \geq T, \text { where } \tilde{M}_{1}(t)=\tilde{M}_{1}(\widehat{2}, t) .
$$

For the proof of (5.35), we combine Theorem 4.4 with the same replacements in Remark 4.8 used for the above derivation of (5.17), and have (4.23) corresponding to the case $\bar{A}(\alpha)<\infty$, have (4.25) corresponding to the case $\bar{A}(\alpha)=\infty, \bar{\beta}(\alpha)<\infty$, and have (4.22) corresponding to the case $\bar{A}(\alpha)=\infty, \bar{\beta}(\alpha)=\infty$.

Similarly, it follows from Theorem 4.3 instead of 4.4 that there is $T_{\alpha}>0$, for $\alpha \geq \widehat{2}$, such that

$$
\sum_{k=1}^{2} \int_{U}\left|\bar{p}_{k}(\cdot, t)\right|^{\alpha} d x \leq C_{\alpha} \tilde{E}(\widehat{\alpha}, t) \text { for all } t \geq T_{\alpha}
$$

where

$$
\tilde{E}(\alpha, t)= \begin{cases}\bar{A}(\alpha) & \text { if } \bar{A}(\alpha)<\infty, \\ \bar{\beta}(\alpha)^{\frac{\alpha}{\alpha-2 a}}+\tilde{\mathcal{A}}(\alpha, t) & \text { if } \bar{A}(\alpha)=\infty, \bar{\beta}(\alpha)<\infty, \\ \operatorname{Env} \tilde{\mathcal{A}}(\alpha, t) & \text { if } \bar{A}(\alpha)=\infty, \bar{\beta}(\alpha)=\infty,\end{cases}
$$

For $\alpha \geq 0$ and $\lambda \geq \widehat{\theta_{2} \alpha}$, by (5.22), (5.35) and (5.38) with $\alpha=\lambda=\widehat{\lambda}$, there is $T_{\alpha, \lambda}>0$ such that

$$
M_{2}(\alpha, t) \leq C_{\alpha, \lambda} \tilde{M}_{2}(\lambda, t) \text { for all } t \geq T_{\alpha, \lambda},
$$

where

$$
\tilde{M}_{2}(\alpha, t)=\tilde{M}_{1}(\alpha, t)+\tilde{E}(\alpha, t) .
$$

Taking $\lambda=\widehat{\theta_{2} \alpha}$, estimate $(5.40)$ reads

$$
M_{2}(\alpha, t) \leq C_{\alpha} \tilde{M}_{2}\left(\widehat{\theta_{2} \alpha}, t\right) \text { for all } t \geq T_{\alpha}, \text { some } T_{\alpha}>0 .
$$

The functions $N(\lambda, t)$ and $F(\alpha, t)$ can be treated similarly to obtain counter parts of (5.31) and (5.33). The counter part of $\bar{N}(\lambda, t)$ is

$$
\tilde{N}(\lambda, t)= \begin{cases}1+\tilde{E}(\widehat{\lambda}, t)^{1 / \widehat{\lambda}} & \text { if } \lambda>0 \\ 1 & \text { if } \lambda=0\end{cases}
$$

the counter part of $\bar{F}(\alpha, t)$ is

$$
\tilde{F}(\alpha, t)=\tilde{N}(\alpha, t)^{\alpha-1}+\tilde{N}\left(\gamma_{1}(\alpha), t\right)^{\alpha-2} \tilde{M}_{1}(t)^{\frac{1-a}{2-a}},
$$

and the counter part of $\bar{F}(\alpha, \lambda, t)$ is

$$
\tilde{F}(\alpha, \lambda, t)=\tilde{N}(\lambda, t)^{\alpha-1}+\tilde{N}(\lambda, t)^{\alpha-2} \tilde{M}_{1}(\lambda, t)^{\frac{1-a}{2-a}} .
$$


For $\alpha \geq 2$, resp. $\alpha \geq 2$ and $\lambda \geq \max \left\{\alpha, \gamma_{1}(\alpha), \widehat{2}\right\}$, using (5.38) we have

$$
\begin{gathered}
N(\alpha, t) \leq C_{\alpha} \tilde{N}(\alpha, t) \text { and } N\left(\gamma_{1}, t\right) \leq C_{\alpha} \tilde{N}\left(\gamma_{1}, t\right), \\
\text { resp. } N(\alpha, t), N\left(\gamma_{1}, t\right) \leq C_{\alpha, \lambda} \tilde{N}(\lambda, t),
\end{gathered}
$$

for sufficiently large $t$, hence combining this with (5.37), resp. with (5.35) for $\alpha$ set to equal $\lambda$, we obtain

$$
\begin{gathered}
F(\alpha, t) \leq C_{\alpha} \tilde{F}(\alpha, t) \text { for all } t \geq T_{\alpha}, \text { some } T_{\alpha}>0, \\
\text { resp. } F(\alpha, t) \leq C_{\alpha, \lambda} \tilde{F}(\alpha, \lambda, t) \text { for all } t \geq T_{\alpha, \lambda}, \text { some } T_{\alpha, \lambda}>0 .
\end{gathered}
$$

Note that the quantities $\tilde{M}_{1}(\alpha, t), \tilde{M}_{2}(\alpha, t), \tilde{N}(\lambda, t), \tilde{F}(\alpha, t)$ and $\tilde{F}(\alpha, \lambda, t)$ depend on the boundary data $\Psi_{1}(x, t)$ and $\Psi_{2}(x, t)$, but are independent of the initial data $p_{1}(x, 0)$ and $p_{2}(x, 0)$.

Throughout, we assume all of the quantities $\mathcal{G}_{j}(t), \tilde{A}(\alpha, t), \bar{M}_{k}(\alpha, t), \tilde{M}_{k}(\alpha, t), \bar{N}(\alpha, t)$, $\tilde{N}(\alpha, t), \bar{F}(\alpha, t), \tilde{F}(\alpha, t), \bar{F}(\alpha, \lambda, t), \tilde{F}(\alpha, \lambda, t)$, as functions of $t$, are continuous on $[0, \infty)$ whenever they are in use.

With the above preparations, we are ready for continuous dependence results.

Theorem 5.2. (i) Assume (DC) and $\alpha \geq 2$. Then

$$
\begin{aligned}
\int_{U}|\bar{z}(x, t)|^{\alpha} d x \leq e^{-c_{8} \int_{0}^{t} \bar{M}_{1}(\tau)^{\frac{-a}{2-a}}} d \tau & \int_{U}|\bar{z}(x, 0)|^{\alpha} d x \\
& +C \int_{0}^{t} e^{-c_{8} \int_{\tau}^{t} \bar{M}_{1}(s)^{\frac{-a}{2-a}} d s} \bar{F}(\alpha, \tau) D(\alpha, \tau) d \tau
\end{aligned}
$$

for all $t \geq 0$, where $c_{8}=c_{8}(\alpha)>0$. Moreover, if $\int_{0}^{\infty} \tilde{M}_{1}(t)^{-\frac{a}{2-a}} d t=\infty$ then

$$
\limsup _{t \rightarrow \infty} \int_{U}|\bar{z}(x, t)|^{\alpha} d x \leq C \limsup _{t \rightarrow \infty}\left[\tilde{F}(\alpha, t) \tilde{M}_{1}(t)^{\frac{a}{2-a}} D(\alpha, t)\right] \text {. }
$$

(ii) Assume (NDC) and $\alpha \geq \alpha_{*}$. Then

$$
\int_{U}|\bar{z}(x, t)|^{\alpha} d x \leq \int_{U}|\bar{z}(x, 0)|^{\alpha} d x+C\left[\operatorname{Env}\left(\bar{F}(\alpha, t) \bar{M}_{2}\left(\widehat{\theta_{2} \alpha}, t\right)^{\frac{2-\theta_{1}}{\theta_{1}}} D(\alpha, t)\right)\right]^{\frac{1}{\theta}}
$$

for all $t \geq 0$. Moreover, if $\int_{0}^{\infty} \tilde{M}_{2}(t)^{-\frac{2-\theta_{1}}{\theta_{1}}} d t=\infty$ then

$$
\limsup _{t \rightarrow \infty} \int_{U}|\bar{z}(x, t)|^{\alpha} d x \leq C \limsup _{t \rightarrow \infty}\left[\tilde{F}(\alpha, t) \tilde{M}_{2}\left(\widehat{\theta_{2} \alpha}, t\right)^{\frac{2-\theta_{1}}{\theta_{1}}} D(\alpha, t)\right]^{\frac{1}{\theta}} .
$$

Proof. (i) By (5.2), estimates (5.19) and (5.31):

$$
\frac{d}{d t} \int_{U}|\bar{z}|^{\alpha} d x \leq-c_{8}\left[\int_{U}|\bar{z}|^{\alpha} d x\right] \bar{M}_{1}(t)^{-\frac{a}{2-a}}+C \bar{F}(\alpha, t) D(\alpha, t) .
$$

Applying Gronwall's inequality we obtain (5.48).

By (5.2), (5.37) and (5.46), we have for $t>T$ that

$$
\frac{d}{d t} \int_{U}|\bar{z}|^{\alpha} d x \leq-C_{1}\left[\int_{U}|\bar{z}|^{\alpha} d x\right] \tilde{M}_{1}(t)^{-\frac{a}{2-a}}+C_{2} \tilde{F}(\alpha, t) D(\alpha, t) .
$$


Then applying estimate (2.34) in Lemma 2.7 with $y(t)=\int_{U}|\bar{z}(x, t)|^{\alpha} d x, h(t)=$ $C_{1} \tilde{M}_{1}(t)^{-\frac{a}{2-a}}, f(t)=C_{2} \tilde{F}(\alpha, t) D(\alpha, t)$ and $\theta=1$ yields (5.49).

(ii) By (5.6), (5.27) and (5.31) we have

$$
\frac{d}{d t} \int_{U}|\bar{z}|^{\alpha} d x \leq-C_{3}\left[\int_{U}|\bar{z}|^{\alpha} d x\right]^{\theta} \bar{M}_{2}\left(\widehat{\theta_{2} \alpha}, t\right)^{-\frac{2-\theta_{1}}{\theta_{1}}}+C_{4} \bar{F}(\alpha, t) D(\alpha, t) .
$$

Applying estimate (2.33) in Lemma 2.7 to (5.52) with $y(t)=\int_{U}|\bar{z}(x, t)|^{\alpha} d x, h(t)=$ $C_{3} \bar{M}_{2}\left(\widehat{\theta_{2} \alpha}, t\right)^{-\frac{2-\theta_{1}}{\theta_{1}}}, f(t)=C_{4} \bar{F}(\alpha, t) D(\alpha, t)$, we obtain $(5.50)$.

Thanks to (5.42) and (5.46), we have for $t>T$ that

$$
\frac{d}{d t} \int_{U}|\bar{z}|^{\alpha} d x \leq-C_{5}\left[\int_{U}|\bar{z}|^{\alpha} d x\right]^{\theta} \tilde{M}_{2}\left(\widehat{\theta_{2} \alpha}, t\right)^{-\frac{2-\theta_{1}}{\theta_{1}}}+C_{6} \tilde{F}(\alpha, t) D(\alpha, t),
$$

Applying (2.34) in Lemma 2.7 to $(5.53)$ on $(T, \infty)$, we obtain $(5.51)$.

According to Theorem 5.2, even when the individual boundary data $\Psi_{1}$ and $\Psi_{2}$, characterized here by $\bar{M}_{k}$ and $\bar{F}$, are asymptotically large as $t \rightarrow \infty$, if their difference $\Phi$, characterized by $D(t)$, is asymptotically sufficiently small to diminish their growth, then $\int_{U}|\bar{z}(x, t)|^{\alpha} d x$ is asymptotically small. For all time estimates, $\int_{U}|\bar{z}(x, t)|^{\alpha} d x$ can be controlled by $\int_{U}|\bar{z}(x, 0)|^{\alpha} d x$ and small $D(t)$. Below, we present a simple scenario.

Corollary 5.3. Let $\alpha \geq \max \left\{2, \alpha_{*}\right\}$. Assume the functions $\Psi_{k}(k=1,2)$ satisfy

$$
\begin{gathered}
\sup _{[0, \infty)}\left\{\left\|\nabla \Psi_{k}(\cdot, t)\right\|_{L^{\infty}},\left\|\left(\Psi_{k}\right)_{t}(\cdot, t)\right\|_{L^{\infty}},\left\|\nabla\left(\Psi_{k}\right)_{t}(\cdot, t)\right\|_{L^{\infty}}\right\}<\infty, \\
\lim _{t \rightarrow \infty}\|\nabla \Phi(\cdot, t)\|_{L^{\gamma}}=\lim _{t \rightarrow \infty}\left\|\Phi_{t}(\cdot, t)\right\|_{L^{\alpha}}=0,
\end{gathered}
$$

where $\gamma=\max \{\alpha, 2(2-a)\}$. Then

$$
\lim _{t \rightarrow \infty} \int_{U}|\bar{z}(x, t)|^{\alpha} d x=0 .
$$

Proof. On the one hand, we have, thanks to (5.54), that $A\left[\Psi_{k}\right](\widehat{2}, t), A\left[\Psi_{k}\right](\widehat{\alpha}, t), A\left[\Psi_{k}\right]\left(\widehat{\theta_{2} \alpha}, t\right)$, $A\left[\Psi_{k}\right]\left(\widehat{\gamma}_{1}, t\right)$ and $G_{3}\left[\Psi_{k}\right](t)$, for $k=1,2$, are uniformly bounded on $[0, \infty)$, hence so are $\tilde{M}_{1}(t), \tilde{M}_{2}\left(\widehat{\theta_{2} \alpha}, t\right)$ and $\tilde{F}(\alpha, t)$. On the other hand, by (5.55) we have $\lim _{t \rightarrow \infty} D(\alpha, t)=0$. Therefore inequality (5.49), resp. (5.51), implies (5.56) in the DC, resp. NDC, case.

We turn to the continuous dependence for the pressure gradient. Because the DC case was treated in [14], we now focus on the NDC case.

Lemma 5.4. In the NDC case, we have for all $t>0$ that

$$
\left[\int_{U}|\nabla z|^{2-a} d x\right]^{\frac{2}{2-a}} \leq C M_{1}(t)^{\frac{1}{2-a}}\|\nabla \Phi(\cdot, t)\|_{L^{2-a}}+C M_{1}(t)^{\frac{a}{2-a}} M_{3}(t)^{\frac{1}{2}}\left[\int_{U}|\bar{z}|^{\alpha_{*}} d x\right]^{\frac{1}{\alpha_{*}}},
$$

where

$$
M_{3}(t)=\int_{U}\left|\left(\bar{p}_{1}\right)_{t}(x, t)\right|^{2}+\left|\left(\bar{p}_{2}\right)_{t}(x, t)\right|^{2} d x+\int_{U}\left|\Phi_{t}(x, t)\right|^{2} d x
$$


Proof. Multiplying the first equation of (5.1) by $\bar{z}$ and integrating over $U$ give

$$
\int_{U} \bar{z} \bar{z}_{t} d x=-\int_{U}\left(K\left(\left|\nabla p_{1}\right|\right) \nabla p_{1}-K\left(\left|\nabla p_{2}\right|\right) \nabla p_{2}\right) \cdot(\nabla z-\nabla \Phi) d x-\int_{U} \Phi_{t} \bar{z} d x .
$$

Hence

$$
\begin{aligned}
& \int_{U}\left(K\left(\left|\nabla p_{1}\right|\right) \nabla p_{1}-K\left(\left|\nabla p_{2}\right|\right) \nabla p_{2}\right) \cdot(\nabla z) d x \\
& \leq \int_{U}\left(K\left(\left|\nabla p_{1}\right|\right)\left|\nabla p_{1}\right|+K\left(\left|\nabla p_{2}\right|\right)\left|\nabla p_{2}\right|\right)|\nabla \Phi| d x+\int_{U}\left(\left|\bar{z}_{t}\right|+\left|\Phi_{t}\right|\right)|\bar{z}| d x .
\end{aligned}
$$

By Lemma 2.1(ii),

$$
\int_{U}\left(K\left(\left|\nabla p_{1}\right|\right) \nabla p_{1}-K\left(\left|\nabla p_{2}\right|\right) \nabla p_{2}\right) \cdot(\nabla z) d x \geq C M_{1}(t)^{\frac{-a}{2-a}}\left(\int_{U}|\nabla z|^{2-a} d x\right)^{\frac{2}{2-a}} .
$$

By relation (2.10),

$$
\begin{aligned}
& \int_{U}\left(K\left(\left|\nabla p_{1}\right|\right)\left|\nabla p_{1}\right|+K\left(\left|\nabla p_{2}\right|\right)\left|\nabla p_{2}\right|\right)|\nabla \Phi| d x \leq C \int_{U}\left(\left|\nabla p_{1}\right|^{1-a}+\left|\nabla p_{2}\right|^{1-a}\right)|\nabla \Phi| d x \\
& \leq C\left(\int_{U}\left|\nabla p_{1}\right|^{2-a}+\left|\nabla p_{1}\right|^{2-a} d x\right)^{\frac{1-a}{2-a}}\left(\int_{U}|\nabla \Phi|^{2-a} d x\right)^{\frac{1}{2-a}} \leq C M_{1}(t)^{\frac{1-a}{2-a}}\|\nabla \Phi\|_{L^{2-a}} .
\end{aligned}
$$

Recall from (2.17) that $\alpha_{*}>2$ in this NDC case. Applying Hölder's inequality and using $\left|\bar{z}_{t}\right| \leq\left|\left(\bar{p}_{1}\right)_{t}\right|+\left|\left(\bar{p}_{2}\right)_{t}\right|$, we have

$$
\begin{aligned}
\int_{U}\left(\left|\bar{z}_{t}\right|+\left|\Phi_{t}\right|\right)|\bar{z}| d x & \leq C\left(\int_{U}\left|\left(\bar{p}_{1}\right)_{t}\right|^{2}+\left|\left(\bar{p}_{2}\right)_{t}\right|^{2}+\left|\Phi_{t}\right|^{2} d x\right)^{\frac{1}{2}}\left(\int_{U}|\bar{z}|^{\alpha_{*}} d x\right)^{\frac{1}{\alpha_{*}}} \\
& \leq C M_{3}(t)^{\frac{1}{2}}\left(\int_{U}|\bar{z}|^{\alpha_{*}} d x\right)^{\frac{1}{\alpha_{*}}} .
\end{aligned}
$$

Combining all the above, we obtain

$$
M_{1}(t)^{-\frac{a}{2-a}}\left(\int_{U}|\nabla z|^{2-a} d x\right)^{\frac{2}{2-a}} \leq C M_{1}(t)^{\frac{1-a}{2-a}}\|\nabla \Phi\|_{L^{2-a}}+C M_{3}(t)^{\frac{1}{2}}\left(\int_{U}|\bar{z}|^{\alpha_{*}} d x\right)^{\frac{1}{\alpha_{*}}} .
$$

Then multiplying by $M_{1}(t)^{\frac{a}{2-a}}$ yields (5.57)

By virtue of Lemma 5.4 we need to estimate $M_{3}(t)$. For each $k=1,2$, applying inequality (4.29) in Theorem 4.4 for $p_{k}$ with $t_{0}=1$ and using Remark 4.8 with $\alpha^{\prime}=\alpha \geq$ $\widehat{2}$ and $G(t)=\tilde{\mathcal{A}}(\alpha, t)$, we have

$$
M_{3}(t) \leq C_{\alpha} \bar{M}_{3}(\alpha, t) \text { for all } t \geq 1
$$

where

$$
\begin{aligned}
& \bar{M}_{3}(\alpha, t)=1+\sum_{k=1}^{2} \int_{U}\left|\bar{p}_{k}(x, 0)\right|^{\alpha}+\left|\nabla p_{k}(x, 0)\right|^{2-a} d x \\
& +\int_{0}^{1} \mathcal{G}_{3}(\tau) d \tau+\int_{0}^{t} e^{-d_{6}(t-\tau)}\left(\operatorname{Env} \tilde{\mathcal{A}}(\alpha, \tau)+\mathcal{G}_{4}(\tau)\right) d \tau+\int_{U}\left|\Phi_{t}(x, t)\right|^{2} d x
\end{aligned}
$$

In particular, when $\alpha=\widehat{2}$

$$
M_{3}(t) \leq C \bar{M}_{3}(t) \text { for all } t \geq 1, \text { where } \bar{M}_{3}(t)=\bar{M}_{3}(\widehat{2}, t) .
$$


For $\alpha \geq \widehat{2}$, same as estimate (5.35) but using Theorem 4.5 (setting $\left.t_{0}=1\right)$ instead of Theorem 4.4 and using the same replacements for Remark 4.8, there exists $T_{\alpha} \geq 1$ such that

$$
M_{3}(t) \leq C_{\alpha} \tilde{M}_{3}(\alpha, t) \text { for all } t \geq T_{\alpha}
$$

where

$$
\begin{aligned}
& \tilde{M}_{3}(\alpha, t)=1+\int_{U}\left|\Phi_{t}(x, t)\right|^{2} d x \\
& + \begin{cases}\bar{A}(\alpha)+\int_{1}^{t} e^{-d_{6}(t-\tau)} \mathcal{G}_{4}(\tau) d \tau & \text { if } \bar{A}(\alpha)<\infty, \\
\bar{\beta}(\alpha)^{\overline{\alpha-2 a}}+\int_{1}^{t} e^{-d_{6}(t-\tau)}\left(\tilde{\mathcal{A}}(\alpha, \tau)+\mathcal{G}_{4}(\tau)\right) d \tau & \text { if } \bar{A}(\alpha)=\infty, \bar{\beta}(\alpha)<\infty, \\
\operatorname{Env} \tilde{\mathcal{A}}(\alpha, t)+\int_{1}^{t} e^{-d_{6}(t-\tau)} \mathcal{G}_{4}(\tau) d \tau & \text { if } \bar{A}(\alpha)=\infty, \bar{\beta}(\alpha)=\infty .\end{cases}
\end{aligned}
$$

Particularly, when $\alpha=\widehat{2}$ there exists $T \geq 1$ such that

$$
M_{3}(t) \leq C \tilde{M}_{3}(t) \text { for all } t \geq T, \text { where } \tilde{M}_{3}(t)=\tilde{M}_{3}(\widehat{2}, t) .
$$

Combining Lemma 5.4 and previous estimates for the quantities $M_{1}(t), M_{2}(t), M_{3}(t)$ and $\int_{U}|\bar{z}(x, t)|^{\alpha_{*}} d x$ will give continuous dependence results for the pressure gradient. We will explicate in details below. Let

$$
\begin{aligned}
\mu_{1} & =\max \left\{2, \alpha_{*}, \theta_{2} \alpha_{*}, \gamma_{1}\left(\alpha_{*}\right)\right\}, \\
\mu_{2} & =\max \left\{\frac{\alpha_{*}-1}{\mu_{1}}, \frac{\alpha_{*}-2}{\mu_{1}}+\frac{1-a}{2-a}\right\}, \\
\mu_{3} & =\frac{a}{2-a}+\frac{1}{2}+\frac{1}{\theta \alpha_{*}}\left(\mu_{2}+\frac{2-\theta_{1}}{\theta_{1}}\right) .
\end{aligned}
$$

Taking $\alpha=\mu_{1}$ in (5.35), taking $\alpha=\alpha_{*}$ and $\lambda=\mu_{1}$ in (5.40), taking $\alpha=\mu_{1}$ in (5.62), and taking $\alpha=\alpha_{*}$ and $\lambda=\mu_{1}$ in (5.47), we assert that there is $T>0$ such that for $t \geq T$,

$$
\begin{gathered}
M_{1}(t) \leq C \tilde{M}_{1}\left(\mu_{1}, t\right), M_{2}\left(\alpha_{*}, t\right) \leq C \tilde{M}_{2}\left(\mu_{1}, t\right), M_{3}(t) \leq C \tilde{M}_{3}\left(\mu_{1}, t\right), \\
F\left(\alpha_{*}, t\right) \leq C \tilde{F}\left(\alpha_{*}, \mu_{1}, t\right) .
\end{gathered}
$$

Theorem 5.5. Assume (NDC).

(i) Then

$$
\left(\int_{U}|\nabla z(x, t)|^{2-a} d x\right)^{\frac{2}{2-a}} \leq C \bar{M}_{1}(t)^{\frac{1}{2-a}}\|\nabla \Phi(\cdot, t)\|_{L^{2-a}}+C \bar{M}_{1}(t)^{\frac{a}{2-a}} \bar{M}_{3}(t)^{\frac{1}{2}} \bar{D}(t)
$$

for all $t \geq 1$, where

$$
\bar{D}(t)=\|\bar{z}(\cdot, 0)\|_{L^{\alpha *}}+\left[\operatorname{Env}\left(\bar{F}\left(\alpha_{*}, t\right) \bar{M}_{2}\left(\widehat{\theta_{2} \alpha_{*}}, t\right)^{\frac{2-\theta_{1}}{\theta_{1}}} D\left(\alpha_{*}, t\right)\right)\right]^{\frac{1}{\theta \alpha_{*}}},
$$

with $\bar{F}\left(\alpha_{*}, t\right), \bar{M}_{2}\left(\widehat{\theta_{2} \alpha_{*}}, t\right)$ and $D\left(\alpha_{*}, t\right)$ defined by (5.32), (5.26) and (5.5), respectively. 
(ii) Let $\eta_{1}=1+\bar{A}\left(\mu_{1}\right)+\lim \sup _{t \rightarrow \infty} \mathcal{G}_{4}(t)$. If $\eta_{1}<\infty$ then

$$
\begin{aligned}
& \limsup _{t \rightarrow \infty}\left(\int_{U}|\nabla z(x, t)|^{2-a} d x\right)^{\frac{2}{2-a}} \leq C \eta_{1}^{\frac{1}{2-a}} \limsup _{t \rightarrow \infty}\|\nabla \Phi(\cdot, t)\|_{L^{2-a}} \\
& \quad+C \eta_{1}^{\mu_{3}} \limsup _{t \rightarrow \infty}\left\{\|\nabla \Phi(\cdot, t)\|_{L^{2(2-a)}}+\|\nabla \Phi(\cdot, t)\|_{L^{\alpha *}}^{2}+\left\|\Phi_{t}(\cdot, t)\right\|_{L^{\alpha *}}\right\}^{\frac{1}{\theta \alpha_{*}}} .
\end{aligned}
$$

Proof. (i) For the last integral of (5.57), we use (5.50) to bound

$$
\left(\int_{U}|\bar{z}|^{\alpha_{*}} d x\right)^{\frac{1}{\alpha_{*}}} \leq C \bar{D}(t) .
$$

Thus (5.67) follows from inequality (5.57) and estimates (5.19), (5.59) and (5.70).

(ii) Note in this NDC case that $\widehat{2}=\alpha_{*}$. By (5.65) and (2.35), we obtain

$$
\begin{cases}\lim \sup _{t \rightarrow \infty} M_{1}(t) & \leq C \lim \sup _{t \rightarrow \infty} \tilde{M}_{1}\left(\mu_{1}, t\right) \leq C \eta_{1}, \\ \limsup _{t \rightarrow \infty} M_{2}\left(\alpha_{*}, t\right) & \leq C \lim \sup _{t \rightarrow \infty} \tilde{M}_{2}\left(\mu_{1}, t\right) \leq C \eta_{1}, \\ \lim \sup _{t \rightarrow \infty} M_{3}(t) & \leq C \lim \sup _{t \rightarrow \infty} \tilde{M}_{3}\left(\mu_{1}, t\right) \leq C \eta_{1} .\end{cases}
$$

Above, for $M_{3}(t)$ we first bound $\int_{U}\left|\Phi_{t}(x, t)\right|^{2} d x$ by $\sum_{k=1,2} \int_{U}\left|\left(\Psi_{k}\right)_{t}(x, t)\right|^{2} d x$ and then absorb it into $\mathcal{G}_{4}(t)$. Note $\gamma_{1}\left(\alpha_{*}\right)>0$ in this case, then

$$
\tilde{N}\left(\mu_{1}, t\right) \equiv 1+\bar{A}\left(\mu_{1}\right)^{\frac{1}{\mu_{1}}} \leq C \eta_{1}^{\frac{1}{\mu_{1}}}
$$

and hence by (5.66),

$$
\limsup _{t \rightarrow \infty} F\left(\alpha_{*}, t\right) \leq C \limsup _{t \rightarrow \infty} \tilde{F}\left(\alpha_{*}, \mu_{1}, t\right) \leq C\left(\eta_{1}^{\frac{\alpha_{*}-1}{\mu_{1}}}+\eta_{1}^{\frac{\alpha_{*}-2}{\mu_{1}}} \eta_{1}^{\frac{1-a}{2-a}}\right) \leq C \eta_{1}^{\mu_{2}} .
$$

Applying (2.34) of Lemma 2.7 to (5.6) yields

$$
\begin{array}{r}
\limsup _{t \rightarrow \infty} \int_{U}|\bar{z}(x, t)|^{\alpha_{*}} d x \leq C\left(\limsup _{t \rightarrow \infty} F\left(\alpha_{*}, t\right) \limsup _{t \rightarrow \infty} M_{2}\left(\alpha_{*}, t\right)^{\frac{2-\theta_{1}}{\theta_{1}}} \limsup _{t \rightarrow \infty} D\left(\alpha_{*}, t\right)\right)^{\frac{1}{\theta}} \\
\leq C\left(\eta_{1}^{\mu_{2}+\frac{2-\theta_{1}}{\theta_{1}}} \limsup _{t \rightarrow \infty} D\left(\alpha_{*}, t\right)\right)^{\frac{1}{\theta}}
\end{array}
$$

Combining (5.71), (5.73) with (5.57) gives

$$
\begin{aligned}
& \limsup _{t \rightarrow \infty}\left(\int_{U}|\nabla z(x, t)|^{2-a} d x\right)^{\frac{2}{2-a}} \leq C \limsup _{t \rightarrow \infty} M_{1}(t)^{\frac{1}{2-a}} \limsup _{t \rightarrow \infty}\|\nabla \Phi(\cdot, t)\|_{L^{2-a}} \\
& \quad+C \limsup _{t \rightarrow \infty} M_{1}(t)^{\frac{a}{2-a}} \limsup _{t \rightarrow \infty} M_{3}(t)^{\frac{1}{2}}\left(\eta_{1}^{\mu_{2}+\frac{2-\theta_{1}}{\theta_{1}}} \limsup _{t \rightarrow \infty} D\left(\alpha_{*}, t\right)\right)^{\frac{1}{\theta \alpha_{*}}} \\
& \leq C \eta_{1}^{\frac{1}{2-a}} \limsup _{t \rightarrow \infty}\|\nabla \Phi(\cdot, t)\|_{L^{2-a}}+C \eta_{1}^{\frac{a}{2-a}} \eta_{1}^{\frac{1}{2}}\left(\eta_{1}^{\mu_{2}+\frac{2-\theta_{1}}{\theta_{1}}} \limsup _{t \rightarrow \infty} D\left(\alpha_{*}, t\right)\right)^{\frac{1}{\theta \alpha_{*}}}
\end{aligned}
$$

hence (5.69) follows, noting that $D\left(\alpha_{*}, t\right)$ is defined by (5.5) with $\alpha=\alpha_{*}>2$.

Next we deal with the case when $\eta_{1}=\infty$. Let

$$
\mu_{4}=\alpha_{*}(\theta-1)\left(\frac{a}{2-a}+\frac{1}{2}\right)+\frac{2-\theta_{1}}{\theta_{1}} \text { and } \mu_{5}=\mu_{4}+\alpha_{*}\left(\frac{a}{2-a}+\frac{1}{2}\right) .
$$


Theorem 5.6. Assume $(N D C)$ and $\eta_{1}=\infty$. Define for $t \geq 0$,

$$
\omega(t)=\left\{\begin{aligned}
1+\bar{A}\left(\mu_{1}\right)+\int_{0}^{t} e^{-d_{7}(t-\tau)} \mathcal{G}_{4}(\tau) d \tau & \text { if } \bar{A}\left(\mu_{1}\right)<\infty, \\
1+\bar{\beta}\left(\mu_{1}\right)^{\frac{\mu_{1}-2 a}{\mu_{1}}}+\tilde{\mathcal{A}}\left(\mu_{1}, t\right) & \\
\quad+\int_{0}^{t} e^{-d_{7}(t-\tau)}\left(\tilde{\mathcal{A}}\left(\mu_{1}, \tau\right)+\mathcal{G}_{4}(\tau)\right) d \tau & \text { if } \bar{A}\left(\mu_{1}\right)=\infty, \bar{\beta}\left(\mu_{1}\right)<\infty, \\
1+\operatorname{Env} \tilde{\mathcal{A}}\left(\mu_{1}, t\right)+\int_{0}^{t} e^{-d_{7}(t-\tau)} \mathcal{G}_{4}(\tau) d \tau & \text { if } \bar{A}\left(\mu_{1}\right)=\infty, \bar{\beta}\left(\mu_{1}\right)=\infty
\end{aligned}\right.
$$

where $d_{7}=\min \left\{d_{5}, d_{6}\right\}$. If $\int_{0}^{\infty} \omega(t)^{-\mu_{4}} d t=\infty$ and

$$
\eta_{2} \stackrel{\text { def }}{=} \limsup _{t \rightarrow \infty}\left[\left(\omega(t)^{\mu_{5}}\right)^{\prime}\right]^{+}<\infty
$$

then

$$
\begin{aligned}
& \limsup _{t \rightarrow \infty}\left(\int_{U}|\nabla z(x, t)|^{2-a} d x\right)^{\frac{2}{2-a}} \leq C \limsup _{t \rightarrow \infty}\left(\omega(t)^{\frac{1}{2-a}}\|\nabla \Phi(\cdot, t)\|_{L^{2-a}}\right) \\
& +C\left[\limsup _{t \rightarrow \infty} \omega(t)^{\mu_{6}} D(t)\right]^{\frac{1}{\theta \alpha_{*}}}+C \eta_{2}^{\frac{1}{\theta \alpha_{*}}}\left[\limsup _{t \rightarrow \infty} \omega(t)^{\mu_{7}} D(t)\right]^{\frac{1}{\theta^{2} \alpha_{*}}},
\end{aligned}
$$

where $\mu_{6}$ and $\mu_{7}$ are positive numbers defined below by (5.79) and (5.82), respectively.

Proof. Same as for (5.71) and (5.72), one can verify that

$$
\tilde{M}_{1}\left(\mu_{1}, t\right), \tilde{M}_{2}\left(\mu_{1}, t\right), \tilde{M}_{3}\left(\mu_{1}, t\right) \leq C \omega(t) \text { and } \tilde{F}\left(\alpha_{*}, \mu_{1}, t\right) \leq C \omega(t)^{\mu_{2}} \text { for all } t \geq 0 .
$$

Then by (5.65) and (5.66), there is $T>1$ such that

$$
M_{1}(t), M_{2}\left(\alpha_{*}, t\right), M_{3}(t) \leq C \omega(t) \text { and } F\left(\alpha_{*}, t\right) \leq C \omega(t)^{\mu_{2}} \text { for all } t \geq T .
$$

Combining (5.57) with estimates in (5.76) we have for $t \geq T$ that

$$
\left(\int_{U}|\nabla z(x, t)|^{2-a} d x\right)^{\frac{2}{2-a}} \leq C \omega(t)^{\frac{1}{2-a}}\|\nabla \Phi(\cdot, t)\|_{L^{2-a}}+C \omega(t)^{\frac{a}{2-a}+\frac{1}{2}} Z(t)^{\frac{1}{\alpha_{*}}},
$$

where $Z(t)=\int_{U}|\bar{z}(x, t)|^{\alpha_{*}} d x$. Hence by setting $W(t)=\omega(t)^{\alpha_{*}\left(\frac{a}{2-a}+\frac{1}{2}\right)}$ we obtain

$$
\left(\int_{U}|\nabla z(x, t)|^{2-a} d x\right)^{\frac{2}{2-a}} \leq C \omega(t)^{\frac{1}{2-a}}\|\nabla \Phi(\cdot, t)\|_{L^{2-a}}+C(W(t) Z(t))^{\frac{1}{\alpha_{*}}}, \quad t \geq T .
$$

We now estimate the limit superior of the product $W(t) Z(t)$ as $t \rightarrow \infty$. As treated in [13], we derive a differential inequality for $W Z$ first. Denote $\tilde{M}_{2}(t)=\tilde{M}_{2}\left(\widehat{\theta_{2} \alpha_{*}}, t\right), \tilde{F}(t)=$ $\tilde{F}\left(\alpha_{*}, t\right)$ and $D(t)=D\left(\alpha_{*}, t\right)$. For $t>T$, setting $\alpha=\alpha_{*}$ in (5.6) and in estimates (5.42), (5.46), we have

$$
\begin{aligned}
& (W Z)^{\prime}=Z^{\prime} W+Z W^{\prime} \leq-C Z^{\theta} \tilde{M}_{2}^{-\frac{2-\theta_{1}}{\theta_{1}}} W+C \tilde{F} D W+Z W^{\prime} \\
& \leq-C\left(W^{1-\theta} \tilde{M}_{2}^{-\frac{2-\theta_{1}}{\theta_{1}}}\right)(W Z)^{\theta}+C W \tilde{F} D+W^{\prime} Z \\
& \leq-C \omega^{-\alpha_{*}(\theta-1)\left(\frac{a}{2-a}+\frac{1}{2}\right)-\frac{2-\theta_{1}}{\theta_{1}}}(W Z)^{\theta}+C \omega^{\mu_{2}+\alpha_{*}\left(\frac{a}{2-a}+\frac{1}{2}\right)} D+C \omega^{\prime} \omega^{\alpha_{*}\left(\frac{a}{2-a}+\frac{1}{2}\right)-1} Z .
\end{aligned}
$$

Thus

$$
(W Z)^{\prime} \leq-C \omega^{-\mu_{4}}(W Z)^{\theta}+C \omega^{\mu_{2}+\alpha_{*}\left(\frac{a}{2-a}+\frac{1}{2}\right)} D+C\left[\omega^{\prime}\right]^{+} \omega^{\alpha_{*}\left(\frac{a}{2-a}+\frac{1}{2}\right)-1} Z .
$$


Since $\int_{0}^{\infty} \omega(t)^{-\mu_{4}} d t=\infty$, applying (2.34) in Lemma 2.7 for $y(t)=W(t+T) Z(t+T)$ gives

$$
\begin{aligned}
& \limsup _{t \rightarrow \infty} W(t) Z(t) \\
& \quad \leq C \limsup _{t \rightarrow \infty}\left(\omega(t)^{\mu_{4}+\mu_{2}+\alpha_{*}\left(\frac{a}{2-a}+\frac{1}{2}\right)} D(t)+\left[\omega^{\prime}(t)\right]^{+} \omega(t)^{\mu_{4}+\alpha_{*}\left(\frac{a}{2-a}+\frac{1}{2}\right)-1} Z(t)\right)^{\frac{1}{\theta}},
\end{aligned}
$$

or

$$
\limsup _{t \rightarrow \infty} W(t) Z(t) \leq C \limsup _{t \rightarrow \infty}\left(\omega(t)^{\mu_{6}} D(t)+\left[\left(\omega(t)^{\mu_{5}}\right)^{\prime}\right]^{+} Z(t)\right)^{\frac{1}{\theta}},
$$

where

$$
\mu_{6}=\mu_{4}+\mu_{2}+\alpha_{*}\left(\frac{a}{2-a}+\frac{1}{2}\right)=\mu_{5}+\mu_{2} .
$$

Then by (5.78) and (5.74)

$$
\limsup _{t \rightarrow \infty} W(t) Z(t) \leq C\left(\limsup _{t \rightarrow \infty} \omega(t)^{\mu_{6}} D(t)\right)^{\frac{1}{\theta}}+C\left(\eta_{2} \limsup _{t \rightarrow \infty} Z(t)\right)^{\frac{1}{\theta}} .
$$

Therefore (5.77) now gives

$$
\begin{array}{r}
\limsup _{t \rightarrow \infty}\left(\int_{U}|\nabla z(x, t)|^{2-a} d x\right)^{\frac{2}{2-a}} \leq C \limsup _{t \rightarrow \infty}\left(\omega(t)^{\frac{1}{2-a}}\left(\int_{U}|\nabla \Phi(x, t)|^{2-a} d x\right)^{\frac{1}{2-a}}\right) \\
+C \limsup _{t \rightarrow \infty}\left[\omega^{\mu_{6}}(t) D(t)\right]^{\frac{1}{\alpha_{*} \theta}}+C \eta_{2}^{\frac{1}{\alpha_{* \theta}}}\left[\limsup _{t \rightarrow \infty} Z(t)\right]^{\frac{1}{\alpha_{*} \theta}} .
\end{array}
$$

Also, by (5.51) and (5.76):

$$
\limsup _{t \rightarrow \infty} Z(t) \leq \limsup _{t \rightarrow \infty}\left[\omega(t)^{\mu_{7}} D(t)\right]^{\frac{1}{\theta}}
$$

where

$$
\mu_{7}=\mu_{2}+\frac{2-\theta_{1}}{\theta_{1}}
$$

(Since $\theta_{1}<2-a$, the numbers $\mu_{4}, \mu_{5}, \mu_{6}$ and $\mu_{7}$ are positive.) We then obtain (5.75) from (5.80) and (5.81). The proof is complete.

\section{DEPENDENCE ON THE FORCHHEIMER POLYNOMIAL}

In this section we study the dependence of solutions of IBVP (4.1) on the coefficients of the Forchheimer polynomial $g(s)$ in (2.2). Let $N \geq 1$, the exponent vector $\vec{\alpha}=\left(0, \alpha_{1}, \ldots, \alpha_{N}\right)$ and the boundary data $\psi(x, t)$ be fixed. For each Forchheimer

polynomial $g(s, \vec{a})$ in class $F P(N, \vec{\alpha})$ we denote $p(x, t ; \vec{a})$ the solution of (4.1) with $K=K(\xi, \vec{a})$ and initial data $p(x, 0, \vec{a})$.

Let $\mathbf{D}$ be a compact subset of $\left\{\vec{a}=\left(a_{0}, a_{1}, \ldots, a_{N}\right): a_{0}, a_{N}>0, a_{1}, \ldots, a_{N-1} \geq 0\right\}$.

Let $g_{1}(s)=g\left(s, \vec{a}^{(1)}\right)$ and $g_{2}(s)=g\left(s, \vec{a}^{(2)}\right)$ be two functions of class $\operatorname{FP}(N, \vec{\alpha})$, where $\vec{a}^{(1)}$ and $\vec{a}^{(2)}$ belong to $\mathrm{D}$. Let $p_{k}=p_{k}\left(x, t ; \vec{a}^{(k)}\right)$ and $\bar{p}_{k}=p_{k}-\Psi$ for $k=1,2$, where $\Psi$ 
is an extension of $\psi$. Then

$$
\left\{\begin{array}{l}
\frac{\partial \bar{p}_{k}}{\partial t}=\nabla \cdot\left(K\left(\left|\nabla p_{k}\right|, \vec{a}^{(k)}\right) \nabla p_{k}\right)-\Psi_{t} \text { in } U \times(0, \infty) \\
\bar{p}_{k}(x, t)=0 \text { on } \Gamma \times(0, \infty)
\end{array}\right.
$$

We denote the difference between $p_{1}$ and $p_{2}$ by $z=p_{1}-p_{2}=\bar{p}_{1}-\bar{p}_{2}$. Then

$$
\left\{\begin{array}{l}
\frac{\partial z}{\partial t}=\nabla \cdot\left(K\left(\left|\nabla p_{1}\right|, \vec{a}^{(1)}\right) \nabla p_{1}\right)-\nabla \cdot\left(K\left(\left|\nabla p_{2}\right|, \vec{a}^{(2)}\right) \nabla p_{2}\right) \text { in } U \times(0, \infty), \\
z(x, t)=0 \text { on } \Gamma \times(0, \infty) .
\end{array}\right.
$$

We use the following notation for convenience in further discussions: let $\vec{x}=\left(x_{1}, x_{2}, \ldots\right)$ and $\vec{x}^{\prime}=\left(x_{1}^{\prime}, x_{2}^{\prime}, \ldots\right)$ be two arbitrary vectors of the same length, including possible length 1 . We denote by $\vec{x} \vee \vec{x}^{\prime}$ and $\vec{x} \wedge \vec{x}^{\prime}$ their maximum and minimum vectors, respectively, with components

$$
\left(\vec{x} \vee \vec{x}^{\prime}\right)_{j}=\max \left\{x_{j}, x_{j}^{\prime}\right\} \text { and }\left(\vec{x} \wedge \vec{x}^{\prime}\right)_{j}=\min \left\{x_{j}, x_{j}^{\prime}\right\}
$$

We will derive estimates for $\int_{U}|z|^{\alpha} d x$ and $\int_{U}|\nabla z|^{2-a} d x$. Those estimates contain different constants that depend on $\vec{a}^{(1)}, \vec{a}^{(2)}, \vec{a}^{(1)} \vee \vec{a}^{(2)}$ or $\vec{a}^{(1)} \wedge \vec{a}^{(2)}$. To simplify those dependences, we define for $\vec{a} \in \mathbf{D}$

$$
\chi(\vec{a})=\max \left\{a_{0}, a_{1}, \ldots, a_{N}, \frac{1}{a_{0}}, \frac{1}{a_{N}}\right\} \in[1, \infty) \text { and set } \hat{\chi}(\mathbf{D})=\max \{\chi(\vec{a}): \vec{a} \in \mathbf{D}\} .
$$

Then $\hat{\chi}(\mathbf{D})$ is a number in $[1, \infty)$. As shown in [13], all constants $d_{j}, c_{j}, C_{j}$ and $C$ appearing in estimates in the previous sections when $\vec{a}$ varies among the vectors $\vec{a}^{(1)}, \vec{a}^{(2)}$, $\vec{a}^{(1)} \vee \vec{a}^{(2)}$ and $\vec{a}^{(1)} \wedge \vec{a}^{(2)}$, can be made independent of $\vec{a}$; they depend only on $n, U, \hat{\chi}(\mathbf{D})$, $\vec{\alpha}, \theta, \theta_{1}$, and possibly $\alpha$. We still denote them by $d_{j}, c_{j}, C_{j}$ and $C$, respectively, in this section.

Let $M_{1}(t)$ and $M_{2}(\alpha, t)$ be defined by (5.3) and (5.7), respectively.

Lemma 6.1. (i) In the DC case, if $\alpha \geq 3$ then for $t>0$,

$$
\frac{d}{d t} \int_{U}|z(x, t)|^{\alpha} d x \leq-c_{9}\left[\int_{U}|z(x, t)|^{\alpha} d x\right] M_{1}(t)^{-\frac{a}{2-a}}+C\left|\vec{a}^{(1)}-\vec{a}^{(2)}\right| R(t),
$$

where $c_{9}=c_{9}(\alpha)>0$ and $R(t)=R(\alpha, t)=1+R_{1}(t)+R_{2}(t)+R_{3}(t)+R_{4}(t)$ with

$$
\begin{aligned}
& R_{1}(t)=R_{1}(\alpha, t)=\int_{U}|\nabla \Psi(x, t)|^{4-2 a}+\left|\Psi_{t}(x, t)\right|^{\alpha} d x \\
& R_{2}(t)=\sum_{k=1}^{2} \int_{U}\left|\nabla p_{k}(x, t)\right|^{2-a}+\left|\left(\bar{p}_{k}\right)_{t}(x, t)\right|^{2} d x \\
& R_{3}(t)=R_{3}(\alpha, t)=\sum_{k=1}^{2} \int_{U}\left|\bar{p}_{k}(x, t)\right|^{\gamma_{2}} d x \\
& R_{4}(t)=R_{4}(\alpha, t)=\sum_{k=1}^{2} \int_{U}\left|\nabla p_{k}(x, t)\right|^{2-a}\left|\bar{p}_{k}(x, t)\right|^{\gamma_{3}} d x \\
& \gamma_{2}=\gamma_{2}(\alpha) \stackrel{\text { def }}{=} \max \{2 \alpha,(4-2 a)(\alpha-2)\}
\end{aligned}
$$




$$
\gamma_{3}=\gamma_{3}(\alpha) \stackrel{\text { def }}{=} \max \left\{\alpha-2, \frac{2-a}{1-a},(2-a)(\alpha-3)\right\} \text {. }
$$

(ii) In the NDC case, if $\alpha \geq \max \left\{3, \alpha_{*}\right\}$ then for $t>0$,

$$
\frac{d}{d t} \int_{U}|z(x, t)|^{\alpha} d x \leq-c_{10}\left[\int_{U}|z(x, t)|^{\alpha} d x\right]^{\theta} M_{2}(\alpha, t)^{-\frac{2-\theta_{1}}{\theta_{1}}}+C\left|\vec{a}^{(1)}-\vec{a}^{(2)}\right| R(t) \text {, (6.6) }
$$

where $c_{10}=c_{10}\left(\alpha, \theta, \theta_{1}\right)>0$.

Proof. Multiplying both sides of the first equation of (6.2) by $\alpha|z|^{\alpha-1} \operatorname{sign}(z)$, integrating over domain $U$, then applying Green's formula, we have

$$
\begin{aligned}
& \frac{1}{\alpha} \frac{d}{d t} \int_{U}|z|^{\alpha} d x \\
& \quad=-(\alpha-1) \int_{U}\left(K\left(\left|\nabla p_{1}\right|, \vec{a}^{(1)}\right) \nabla p_{1}-K\left(\left|\nabla p_{2}\right|, \vec{a}^{(2)}\right) \nabla p_{2}\right) \cdot\left(\nabla p_{1}-\nabla p_{2}\right)|z|^{\alpha-2} d x .
\end{aligned}
$$

According to the perturbed monotonicity, c.f. Lemma 5.2 in [13], we have

$$
\begin{aligned}
& \left(K\left(\left|\nabla p_{1}\right|, \vec{a}^{(1)}\right) \nabla p_{1}-K\left(\left|\nabla p_{2}\right|, \vec{a}^{(2)}\right) \nabla p_{2}\right) \cdot\left(\nabla p_{1}-\nabla p_{2}\right) \\
& \geq(1-a) K\left(\left|\nabla p_{1}\right| \vee\left|\nabla p_{2}\right|, \vec{a}^{(1)} \vee \vec{a}^{(2)}\right)\left|\nabla p_{1}-\nabla p_{2}\right|^{2} \\
& \quad-C\left|\vec{a}^{(1)}-\vec{a}^{(2)}\right| K\left(\left|\nabla p_{1}\right| \vee\left|\nabla p_{2}\right|, \vec{a}^{(1)} \wedge \vec{a}^{(2)}\right)\left(\left|\nabla p_{1}\right| \vee\left|\nabla p_{2}\right|\right)\left|\nabla p_{1}-\nabla p_{2}\right| .
\end{aligned}
$$

Thus

$$
\frac{d}{d t} \int_{U}|z|^{\alpha} d x \leq-C J_{1}+C\left|\vec{a}^{(1)}-\vec{a}^{(2)}\right| J_{2}
$$

where

$$
\begin{aligned}
& J_{1}=\int_{U} K\left(\left|\nabla p_{1}\right| \vee\left|\nabla p_{2}\right|, \vec{a}^{(1)} \vee \vec{a}^{(2)}\right)|\nabla z|^{2}|z|^{\alpha-2} d x \\
& J_{2}=\int_{U} K\left(\left|\nabla p_{1}\right| \vee\left|\nabla p_{2}\right|, \vec{a}^{(1)} \wedge \vec{a}^{(2)}\right)\left(\left|\nabla p_{1}\right| \vee\left|\nabla p_{2}\right|\right)\left|\nabla p_{1}-\nabla p_{2}\right||z|^{\alpha-2} d x .
\end{aligned}
$$

Regarding $J_{2}$, we have

$$
J_{2} \leq C \int_{U} K\left(\left|\nabla p_{1}\right| \vee\left|\nabla p_{2}\right|, \vec{a}^{(1)} \wedge \vec{a}^{(2)}\right)\left(\left|\nabla p_{1}\right| \vee\left|\nabla p_{2}\right|\right)^{2}|z|^{\alpha-2} d x
$$

then by (2.11),

$$
J_{2} \leq C \int_{U}\left(\left|\nabla p_{1}\right| \vee\left|\nabla p_{2}\right|\right)^{2-a}|\bar{z}|^{\alpha-2} d x \leq C \int_{U}\left(\left|\nabla p_{1}\right|+\left|\nabla p_{2}\right|\right)^{2-a}\left(\left|\bar{p}_{1}\right|^{\alpha-2}+\left|\bar{p}_{2}\right|^{\alpha-2}\right) d x
$$

Hence

$$
J_{2} \leq C\left(L_{1}+L_{2}\right)
$$

where

$$
\begin{aligned}
& L_{1}=\int_{U}\left|\nabla p_{1}\right|^{2-a}\left|\bar{p}_{1}\right|^{\alpha-2} d x+\int_{U}\left|\nabla p_{2}\right|^{2-a}\left|\bar{p}_{2}\right|^{\alpha-2} d x, \\
& L_{2}=\int_{U}\left|\nabla p_{1}\right|^{2-a}\left|\bar{p}_{2}\right|^{\alpha-2} d x+\int_{U}\left|\nabla p_{2}\right|^{2-a}\left|\bar{p}_{1}\right|^{\alpha-2} d x .
\end{aligned}
$$


We estimate the crossed term $L_{2}$. Multiplying the first equation of (6.1) for $\bar{p}_{1}$ by $\bar{p}_{1}\left|\bar{p}_{2}\right|^{\alpha-2}$, multiplying the first equation of (6.1) for $\bar{p}_{2}$ by $\bar{p}_{2}\left|\bar{p}_{1}\right|^{\alpha-2}$, summing up and integrating over domain $U$, we have

$$
\begin{aligned}
& \int_{U}\left(\bar{p}_{1}\right)_{t} \bar{p}_{1}\left|\bar{p}_{2}\right|^{\alpha-2} d x+\int_{U}\left(\bar{p}_{2}\right)_{t} \bar{p}_{2}\left|\bar{p}_{1}\right|^{\alpha-2} d x \\
& =-\int_{U} K\left(\left|\nabla p_{1}\right|, \vec{a}^{(1)}\right) \nabla p_{1} \cdot\left(\nabla p_{1}-\nabla \Psi\right)\left|\bar{p}_{2}\right|^{\alpha-2} d x \\
& \quad-\int_{U} K\left(\left|\nabla p_{2}\right|, \vec{a}^{(2)}\right) \nabla p_{2} \cdot\left(\nabla p_{2}-\nabla \Psi\right)\left|\bar{p}_{1}\right|^{\alpha-2} d x \\
& \quad-(\alpha-2) \int_{U} K\left(\left|\nabla p_{1}\right|, \vec{a}^{(1)}\right)\left(\nabla p_{1} \cdot \nabla \bar{p}_{2}\right) \bar{p}_{1}\left|\bar{p}_{2}\right|^{\alpha-3} \operatorname{sign}\left(\bar{p}_{2}\right) d x \\
& \quad-(\alpha-2) \int_{U} K\left(\left|\nabla p_{2}\right|, \vec{a}^{(2)}\right)\left(\nabla p_{2} \cdot \nabla \bar{p}_{1}\right) \bar{p}_{2}\left|\bar{p}_{1}\right|^{\alpha-3} \operatorname{sign}\left(\bar{p}_{1}\right) d x \\
& \quad-\int_{U} \Psi_{t}\left(\bar{p}_{1}\left|\bar{p}_{2}\right|^{\alpha-2}+\bar{p}_{2}\left|\bar{p}_{1}\right|^{\alpha-2}\right) d x \\
& \leq-I+I_{1}+(\alpha-2) I_{2}+I_{3},
\end{aligned}
$$

where

$$
\begin{aligned}
I & =\int_{U} K\left(\left|\nabla p_{1}\right|, \vec{a}^{(1)}\right)\left|\nabla p_{1}\right|^{2}\left|\bar{p}_{2}\right|^{\alpha-2}+K\left(\left|\nabla p_{2}\right|, \vec{a}^{(2)}\right)\left|\nabla p_{2}\right|^{2}\left|\bar{p}_{1}\right|^{\alpha-2} d x, \\
I_{1} & =\int_{U} K\left(\left|\nabla p_{1}\right|, \vec{a}^{(1)}\right)\left|\nabla p_{1}\right||\nabla \Psi|\left|\bar{p}_{2}\right|^{\alpha-2}+K\left(\left|\nabla p_{2}\right|, \vec{a}^{(2)}\right)\left|\nabla p_{2}\right||\nabla \Psi|\left|\bar{p}_{1}\right|^{\alpha-2} d x, \\
I_{2} & =\int_{U} K\left(\left|\nabla p_{1}\right|, \vec{a}^{(1)}\right)\left|\nabla p_{1}\right|\left|\nabla \bar{p}_{2}\right|\left|\bar{p}_{1}\right|\left|\bar{p}_{2}\right|^{\alpha-3}+K\left(\left|\nabla p_{2}\right|, \vec{a}^{(2)}\right)\left|\nabla p_{2}\right|\left|\nabla \bar{p}_{1}\right|\left|\bar{p}_{2}\right|\left|\bar{p}_{1}\right|^{\alpha-3} d x, \\
I_{3} & =\int_{U}\left|\Psi_{t}\right|\left|\bar{p}_{1}\right|\left|\bar{p}_{2}\right|^{\alpha-2}+\left|\Psi_{t}\right|\left|\bar{p}_{2}\right|\left|\bar{p}_{1}\right|^{\alpha-2} d x .
\end{aligned}
$$

Hence

$$
I \leq I_{1}+(\alpha-2) I_{2}+I_{3}+I_{4}
$$

where

$$
I_{4}=\int_{U}\left|\left(\bar{p}_{1}\right)_{t}\right|\left|\bar{p}_{1}\right|\left|\bar{p}_{2}\right|^{\alpha-2}+\left|\left(\bar{p}_{2}\right)_{t}\right|\left|\bar{p}_{2}\right|\left|\bar{p}_{1}\right|^{\alpha-2} d x
$$

By (2.11) we have

$$
\begin{aligned}
I & \left.\geq C\left[\int_{U}\left|\nabla p_{1}\right|^{2-a}-1\right)\left|\bar{p}_{2}\right|^{\alpha-2} d x+\int_{U}\left(\left|\nabla p_{2}\right|^{2-a}-1\right)\left|\bar{p}_{1}\right|^{\alpha-2} d x\right] \\
& =C\left[L_{2}-\int_{U}\left|\bar{p}_{1}\right|^{\alpha-2}+\left|\bar{p}_{2}\right|^{\alpha-2} d x\right] .
\end{aligned}
$$

Together with (6.12) we have

$$
\begin{aligned}
L_{2} & \leq C I+C\left[\int_{U}\left|\bar{p}_{1}\right|^{\alpha-2}+\left|\bar{p}_{2}\right|^{\alpha-2} d x\right] \\
& \leq C\left[\int_{U}\left|\bar{p}_{1}\right|^{\alpha-2}+\left|\bar{p}_{2}\right|^{\alpha-2} d x+I_{1}+I_{2}+I_{3}+I_{4}\right] .
\end{aligned}
$$


- Estimating $I_{1}$ : Applying Young's inequality to three functions $\left|\nabla p_{k}\right|^{1-a},|\nabla \Psi|,\left|\bar{p}_{3-k}\right|^{\alpha-2}$, for $k=1,2$, with powers $\frac{2-a}{1-a}, 2(2-a), 2(2-a)$ respectively, we have

$$
\begin{aligned}
I_{1} & \leq C \int_{U}\left|\nabla p_{1}\right|^{1-a}|\nabla \Psi|\left|\bar{p}_{2}\right|^{\alpha-2} d x+\int_{U}\left|\nabla p_{2}\right|^{1-a}|\nabla \Psi|\left|\bar{p}_{1}\right|^{\alpha-2} d x \\
& \leq C \sum_{k=1}^{2}\left[\int_{U}\left|\nabla p_{k}\right|^{2-a}+|\nabla \Psi|^{4-2 a}+\left|\bar{p}_{3-k}\right|^{(4-2 a)(\alpha-2)} d x\right] .
\end{aligned}
$$

- Estimating $I_{2}$ : Applying Young's inequality to two functions $\left|\nabla p_{k}\right|^{1-a}\left|\bar{p}_{k}\right|$ and $\left|\nabla \bar{p}_{3-k}\right|\left|\bar{p}_{3-k}\right|^{\alpha-3}$, for $k=1,2$, with powers $\frac{2-a}{1-a}$ and $2-a$, respectively, we have

$$
\begin{aligned}
I_{2} & \leq C\left[\int_{U}\left|\nabla p_{1}\right|^{1-a}\left|\bar{p}_{1}\right|\left|\nabla \bar{p}_{2}\right|\left|\bar{p}_{2}\right|^{\alpha-3} d x+\int_{U}\left|\nabla p_{2}\right|^{1-a}\left|\bar{p}_{2}\right|\left|\nabla \bar{p}_{1}\right|\left|\bar{p}_{1}\right|^{\alpha-3} d x\right] \\
& \leq C \sum_{k=1}^{2}\left[\int_{U}\left|\nabla p_{k}\right|^{2-a}\left|\bar{p}_{k}\right|^{\frac{2-a}{1-a}}+\left|\nabla \bar{p}_{3-k}\right|^{2-a}\left|\bar{p}_{3-k}\right|^{(2-a)(\alpha-3)} d x\right] \\
& \leq C \sum_{k=1}^{2}\left[\int_{U}\left|\nabla p_{k}\right|^{2-a}\left|\bar{p}_{k}\right|^{\frac{2-a}{1-a}}+\left|\nabla p_{3-k}\right|^{2-a}\left|\bar{p}_{3-k}\right|^{(2-a)(\alpha-3)}+|\nabla \Psi|^{2-a}\left|\bar{p}_{3-k}\right|^{(2-a)(\alpha-3)} d x\right] .
\end{aligned}
$$

Applying Cauchy's inequality to the last product gives

$$
\begin{aligned}
I_{2} \leq & C \sum_{k=1}^{2}\left[\int_{U}\left|\nabla p_{k}\right|^{2-a}\left(\left|\bar{p}_{k}\right|^{\frac{2-a}{1-a}}+\left|\bar{p}_{k}\right|^{(2-a)(\alpha-3)}\right)+\left|\bar{p}_{k}\right|^{(4-2 a)(\alpha-3)} d x\right] \\
& +C \int_{U}|\nabla \Psi|^{4-2 a} d x .
\end{aligned}
$$

- Estimating $I_{3}$ : Similarly, applying Young's inequality to three functions $\left|\Psi_{t}\right|,\left|\bar{p}_{k}\right|$, $\left|\bar{p}_{3-k}\right|^{\alpha-2}$, for $k=1,2$, with powers $\alpha, \alpha, \frac{\alpha}{\alpha-2}$ respectively, we have

$$
I_{3} \leq C \sum_{k=1}^{2}\left[\int_{U}\left|\Psi_{t}\right|^{\alpha}+\left|\bar{p}_{k}\right|^{\alpha}+\left|\bar{p}_{3-k}\right|^{\alpha} d x\right]=C \int_{U}\left|\Psi_{t}\right|^{\alpha} d x+C \sum_{k=1}^{2} \int_{U}\left|\bar{p}_{k}\right|^{\alpha} d x .
$$

- Estimating $I_{4}$ : Applying Young's inequality to three functions $\left|\left(\bar{p}_{k}\right)_{t}\right|,\left|\bar{p}_{k}\right|,\left|\bar{p}_{3-k}\right|^{\alpha-2}$, for $k=1,2$, with powers $2, \alpha, \frac{2 \alpha}{\alpha-2}$ respectively, we have

$$
I_{4} \leq C \sum_{k=1}^{2}\left[\int_{U}\left|\left(\bar{p}_{k}\right)_{t}\right|^{2}+\left|\bar{p}_{k}\right|^{\alpha}+\left|\bar{p}_{3-k}\right|^{2 \alpha} d x\right] .
$$

From (6.10), (6.13) and (6.14)-(6.17) we have

$$
\begin{aligned}
L_{1}+L_{2} \leq & C \sum_{k=1}^{2} \int_{U}\left|\bar{p}_{k}\right|^{\alpha-2}+\left|\bar{p}_{k}\right|^{\alpha}+\left|\bar{p}_{k}\right|^{2 \alpha}+\left|\bar{p}_{k}\right|^{(4-2 a)(\alpha-3)}+\left|\bar{p}_{k}\right|^{(4-2 a)(\alpha-2)} d x \\
& +C \sum_{k=1}^{2} \int_{U}\left|\nabla p_{k}\right|^{2-a}\left(1+\left|\bar{p}_{k}\right|^{\alpha-2}+\left|\bar{p}_{k}\right|^{\frac{2-a}{1-a}}+\left|\bar{p}_{k}\right|^{(2-a)(\alpha-3)}\right)+\left|\left(\bar{p}_{k}\right)_{t}\right|^{2} d x \\
& +C \int_{U}|\nabla \Psi|^{4-2 a}+\left|\Psi_{t}\right|^{\alpha} d x .
\end{aligned}
$$


By Young's inequality, we then obtain

$$
\begin{aligned}
L_{1}+L_{2} \leq & C \sum_{k=1}^{2} \int_{U} 1+\left|\bar{p}_{k}\right|^{\gamma_{2}} d x+C \sum_{k=1}^{2} \int_{U}\left|\nabla p_{k}\right|^{2-a}\left(1+\left|\bar{p}_{k}\right|^{\gamma_{3}}\right)+\left|\left(\bar{p}_{k}\right)_{t}\right|^{2} d x \\
& +C \int_{U}|\nabla \Psi|^{4-2 a}+\left|\Psi_{t}\right|^{\alpha} d x
\end{aligned}
$$

thus

$$
L_{1}+L_{2} \leq C R(t)
$$

Combining (6.8), (6.9) and (6.18) gives

$$
\frac{d}{d t} \int_{U}|z|^{\alpha} d x \leq-C J_{1}+C\left|\vec{a}^{(1)}-\vec{a}^{(2)}\right| R(t)
$$

We now use Lemma 2.5 to estimate $J_{1}$. Note that $z=\bar{z}$ in this case.

(i) In the DC case, for $\alpha \geq 3$, we have similar to (5.13) that

$$
-J_{1} \leq-C_{1}\left[\int_{U}|z|^{\alpha} d x\right] M_{1}(t)^{-\frac{a}{2-a}}
$$

Hence combining (6.19) and (6.20) yields (6.3).

(ii) In the NDC case, for $\alpha \geq \max \left\{3, \alpha_{*}\right\}$, similar to (5.14) we have

$$
-J_{1} \leq-C_{1}\left[\int_{U}|z|^{\alpha} d x\right]^{\theta} M_{2}(\alpha, t)^{-\frac{2-\theta_{1}}{\theta_{1}}}
$$

Then (6.6) follows (6.19) and (6.21). The proof is complete.

By Hölder's and Young's inequalities, we easily see that

$$
A(\alpha, t) \leq C\left(1+A\left(\alpha^{\prime}, t\right)\right) \text { for } \alpha<\alpha^{\prime} .
$$

We obtain a dependence result for finite time intervals.

Theorem 6.2. If $\alpha \geq \max \left\{3, \alpha_{*}\right\}$ and $T>0$ then

$$
\int_{U}|z(x, t)|^{\alpha} d x \leq \int_{U}|z(x, 0)|^{\alpha} d x+C M_{0, T}\left|\vec{a}^{(1)}-\vec{a}^{(2)}\right| \text { for all } t \in[0, T],
$$

where

$$
M_{0, T}=1+\sum_{k=1}^{2} \int_{U}\left|\bar{p}_{k}(x, 0)\right|^{\gamma_{4}}+\left|\nabla p_{k}(x, 0)\right|^{2-a} d x+\int_{0}^{T} 1+R_{1}(\tau)+A\left(\gamma_{4}, \tau\right)+G_{3}(\tau) d \tau \text {, }
$$

with $\gamma_{4}=\gamma_{4}(\alpha) \stackrel{\text { def }}{=} \max \left\{\gamma_{2}+a, \gamma_{3}+2\right\}$.

Proof. Dropping the negative term on the right-hand side of inequality (6.3) in the DC case, or of inequality (6.6) in the NDC case, then integrating from 0 to $t$, we have

$$
\int_{U}|z(x, t)|^{\alpha} d x \leq \int_{U}|z(x, 0)|^{\alpha} d x+C\left|\vec{a}^{(1)}-\vec{a}^{(2)}\right| \int_{0}^{t} R(\tau) d \tau
$$

We estimate each integral $\int_{0}^{t} R_{j}(\tau) d \tau$, for $j=2,3,4$, in $\int_{0}^{t} R(\tau) d \tau$. 
- Using relation (2.13), estimates (4.19) and (4.20), we have for $t \geq 0$ that

$$
\begin{aligned}
& \int_{0}^{t} R_{2}(\tau) d \tau=\sum_{k=1}^{2} \int_{0}^{t} \int_{U}\left|\left(\bar{p}_{k}\right)_{t}\right|^{2}+\left|\nabla p_{k}\right|^{2-a} d x d \tau \\
& \leq C+C \sum_{k=1}^{2} \int_{U}\left|\nabla p_{k}(x, 0)\right|^{2-a}+\left|\bar{p}_{k}(x, 0)\right|^{2} d x+C \int_{0}^{t} 1+G_{3}(\tau)+G_{1}(\tau) d \tau .
\end{aligned}
$$

Since $G_{1}(t) \leq G_{3}(t)$, above inequality becomes

$$
\int_{0}^{t} R_{2}(\tau) d \tau \leq C+C \sum_{k=1}^{2} \int_{U}\left|\nabla p_{k}(x, 0)\right|^{2-a}+\left|\bar{p}_{k}(x, 0)\right|^{2} d x+C \int_{0}^{t} 1+G_{3}(\tau) d \tau .
$$

- Observe that

$$
\int_{U}\left|\bar{p}_{k}\right|^{\gamma_{2}} d x \leq C\left(\int_{U}\left|\bar{p}_{k}\right|^{\gamma_{2}+a} d x\right)^{\frac{\gamma_{2}}{\gamma_{2}+a}}=C\left(\int_{U}\left|\bar{p}_{k}\right|^{\gamma_{2}+a} d x\right)^{\frac{1}{\gamma_{0}\left(\gamma_{2}+a\right)}} .
$$

Applying (4.41) with $\alpha=\gamma_{2}+a$ yields

$$
\begin{aligned}
\int_{0}^{t} R_{3}(\tau) d \tau & \leq C \sum_{k=1}^{2} \int_{0}^{t}\left(\int_{U}\left|\bar{p}_{k}(x, \tau)\right|^{\gamma_{2}+a} d x\right)^{\frac{1}{\gamma_{0}\left(\gamma_{2}+a\right)}} d \tau \\
& \leq C \sum_{k=1}^{2} \int_{U}\left|\bar{p}_{k}(x, 0)\right|^{\gamma_{2}+a} d x+C \int_{0}^{t} 1+A\left(\gamma_{2}+a, \tau\right) d \tau
\end{aligned}
$$

- Note that $\gamma_{3}+2 \geq \max \left\{2, \alpha_{*}\right\}$. Applying inequality (4.37) in Corollary 4.7 with $\alpha=\gamma_{3}+2$ gives

$$
\int_{0}^{t} R_{4}(\tau) d \tau \leq C \sum_{k=1}^{2} \int_{U}\left|\bar{p}_{k}(x, 0)\right|^{\gamma_{3}+2} d x+C \int_{0}^{t} 1+A\left(\gamma_{3}+2, \tau\right) d \tau .
$$

Summing up (6.26), (6.27) and (6.28), and using Young's inequality as well as relation (6.22), we obtain for $t \in[0, T]$ that

$$
\begin{aligned}
\int_{0}^{t} R(\tau) d \tau \leq \int_{0}^{T} R(\tau) d \tau \leq & C+C \sum_{k=1}^{2}\left\{\int_{U}\left|\nabla p_{k}(x, 0)\right|^{2-a}+\left|\bar{p}_{k}(x, 0)\right|^{\gamma_{4}} d x\right\} \\
& +C \int_{0}^{T} 1+R_{1}(\tau)+G_{3}(\tau)+A\left(\gamma_{4}, \tau\right) d \tau
\end{aligned}
$$

hence $\int_{0}^{t} R(\tau) d \tau \leq C M_{0, T}$. This and (6.25) prove inequality (6.23).

We now estimate $\int_{U}|z(x, t)|^{\alpha} d x$ for large $t$, the case that is not covered well by Theorem 6.2. As indicated by differential inequalities in Lemma 6.1, we need to bound $M_{1}(t)$, $M_{2}(\alpha, t)$ and $R(t)$. The first two quantities were estimated in section 5 with the use of the function $\tilde{\mathcal{A}}(\alpha, t)$, see (5.16). Taking $\Psi_{1}=\Psi_{2}=\Psi$, we recast $\tilde{\mathcal{A}}(\alpha, t)$ as

$$
\tilde{A}(\alpha, t)=\int_{U}|\nabla \Psi|^{\alpha} d x+\left(\int_{U}\left|\Psi_{t}\right|^{\alpha} d x\right)^{\frac{1}{1-a}}
$$


and have, the same as (5.15),

$$
A(\alpha, t) \leq 1+A(\alpha, t)^{\gamma_{0}(\alpha)} \leq C(1+\tilde{A}(\alpha, t)) .
$$

By Young's inequality,

$$
\tilde{A}(\alpha, t) \leq C_{\alpha, \alpha^{\prime}}\left(1+\tilde{A}\left(\alpha^{\prime}, t\right)\right) \text { for } \alpha<\alpha^{\prime} .
$$

The estimates (5.19) and (5.27) can be rewritten as

$$
M_{1}(t) \leq C \bar{M}_{1}(t) \text { and } M_{2}(t) \leq C \bar{M}_{2}(t) \text { for all } t \geq 0
$$

where

$$
\begin{gathered}
\bar{M}_{1}(t)=1+\sum_{k=1}^{2}\left[\int_{U} \mid \bar{p}_{k}\left(x,\left.0\right|^{2} d x+e^{-d_{5} t} \int_{U}\left|\nabla p_{k}(x, 0)\right|^{2-a} d x\right]\right. \\
+\int_{0}^{t} e^{-d_{5}(t-\tau)}\left(\operatorname{Env} \tilde{A}(\widehat{2}, \tau)+G_{3}(\tau)\right) d \tau \\
\bar{M}_{2}(t) \stackrel{\operatorname{def}}{=} \bar{M}_{2}\left(\widehat{\theta_{2} \alpha}, t\right)=1+\sum_{k=1}^{2}\left[\int_{U} \mid \bar{p}_{k}\left(x,\left.0\right|^{\widehat{\theta_{2} \alpha}} d x+e^{-d_{5} t} \int_{U}\left|\nabla p_{k}(x, 0)\right|^{2-a} d x\right]\right. \\
\quad+\operatorname{Env} \tilde{A}\left(\widehat{\theta_{2} \alpha}, t\right)+\int_{0}^{t} e^{-d_{5}(t-\tau)} G_{3}(\tau) d \tau .
\end{gathered}
$$

We now estimate $R(t)$. Let $\alpha \geq \max \left\{3, \alpha_{*}\right\}$. Applying (4.38) in Corollary 4.7 with $\alpha-2=\gamma_{3}$, we have

$$
\int_{U}\left|\nabla p_{k}\right|^{2-a}\left|\bar{p}_{k}\right|^{\gamma_{3}} \leq C \int_{U}\left|\left(\bar{p}_{k}\right)_{t}\right|^{2} d x+C\left(1+A\left(\gamma_{3}+2, t\right)+\int_{U}\left|\bar{p}_{k}\right|^{2\left(\gamma_{3}+1\right)} d x\right), \quad k=1,2 .
$$

Hence

$$
\begin{aligned}
R(t) \leq & R_{1}(t)+C\left(1+A\left(\gamma_{3}+2, t\right)\right) \\
& +C \sum_{k=1}^{2}\left\{\int_{U}\left|\left(\bar{p}_{k}\right)_{t}\right|^{2}+\left|\nabla p_{k}\right|^{2-a} d x+\int_{U}\left|\bar{p}_{k}\right|^{2\left(\gamma_{3}+1\right)} d x+\left|\bar{p}_{k}\right|^{\gamma_{2}} d x\right\} .
\end{aligned}
$$

By Young's inequality, we obtain for $t>0$ that

$$
R(t) \leq R_{1}(t)+C\left\{1+A\left(\gamma_{3}+2, t\right)+\sum_{k=1}^{2}\left(\int_{U}\left|\bar{p}_{k}\right|^{\gamma_{5}} d x+\int_{U}\left|\left(\bar{p}_{k}\right)_{t}\right|^{2}+\left|\nabla p_{k}\right|^{2-a} d x\right)\right\},
$$

where $\gamma_{5}=\gamma_{5}(\alpha)=\max \left\{\gamma_{2}, 2 \gamma_{3}+2\right\}$.

Using relation (2.13) and using (4.29) with $t_{0}=1$, we have for $t \geq 1$ that

$$
\begin{aligned}
\int_{U}\left|\nabla p_{k}\right|^{2-a}+\left|\left(\bar{p}_{k}\right)_{t}\right|^{2} d x \leq & C\left(1+\int_{U}\left|\bar{p}_{k}(x, 0)\right|^{\widehat{2}}+\left|\nabla p_{k}(x, 0)\right|^{2-a} d x+\int_{0}^{1} G_{3}(\tau) d \tau\right) \\
& +C \int_{0}^{t} e^{-d_{6}(t-\tau)}\left(\operatorname{Env} \tilde{A}(\widehat{2}, \tau)+G_{4}(\tau)\right) d \tau
\end{aligned}
$$


Note that $\gamma_{5} \geq \gamma_{2} \geq 2 \alpha \geq \widehat{2}$. By Theorem 4.3(i),

$$
\int_{U}\left|\bar{p}_{k}\right|^{\gamma_{5}} d x \leq C\left\{1+\int_{U}\left|\bar{p}_{k}(x, 0)\right|^{\gamma_{5}} d x+\operatorname{Env} \tilde{A}\left(\gamma_{5}, t\right)\right\} \text {. }
$$

Summing up, using (6.35) and Young's inequality with $\gamma_{5} \geq \widehat{2}$, we obtain

$$
\begin{aligned}
R(t) & \leq C\left\{1+\sum_{k=1}^{2} \int_{U}\left|\bar{p}_{k}(x, 0)\right|^{\gamma_{5}}+\left|\nabla p_{k}(x, 0)\right|^{2-a} d x+\int_{0}^{1} G_{3}(\tau) d \tau\right\} \\
& +R_{1}(t)+C\left(A\left(\gamma_{3}+2, t\right)+\operatorname{Env} \tilde{A}(\widehat{2}, t)+\operatorname{Env} \tilde{A}\left(\gamma_{5}, t\right)\right)+C \int_{0}^{t} e^{-d_{6}(t-\tau)} G_{4}(\tau) d \tau .
\end{aligned}
$$

Therefore

$$
R(t) \leq C \bar{R}(t), \quad t \geq 1
$$

where

$$
\bar{R}(t)=\bar{R}(\alpha, t) \stackrel{\text { def }}{=} M_{0}+\int_{0}^{1} G_{3}(\tau) d \tau+R_{1}(t)+\operatorname{Env} \tilde{A}\left(\gamma_{5}, t\right)+\int_{0}^{t} e^{-d_{6}(t-\tau)} G_{4}(\tau) d \tau
$$

with

$$
M_{0}=1+\sum_{k=1}^{2}\left\{\int_{U}\left|\bar{p}_{k}(x, 0)\right|^{\gamma_{5}}+\left|\nabla p_{k}(x, 0)\right|^{2-a} d x\right\} .
$$

To avoid complicated expressions, we continue to estimate $\bar{R}(t)$ more explicitly in terms of $\Psi(x, t)$. Let $t \geq 1$. Note that $\int_{0}^{1} G_{3}(t) d t \leq\left(E n v G_{4}\right)(1) \leq\left(E n v G_{4}\right)(t)$ and, similar to (5.24), the last integral of (6.37) is bounded by $d_{6}^{-1} E n v G_{4}(t)$. Therefore

$$
\bar{R}(t) \leq C\left(M_{0}+R_{1}(t)+\operatorname{Env} \tilde{A}\left(\gamma_{5}, t\right)+\operatorname{Env}_{4}(t)\right) .
$$

To unify different dependences on $\Psi(x, t)$ of the terms on the right-hand side of (6.39), we introduce, for $\gamma>0$, the function

$$
S(\gamma, t)=\int_{U}|\nabla \Psi(x, t)|^{\gamma}+\left(\int_{U}\left|\Psi_{t}(x, t)\right|^{\gamma} d x\right)^{\frac{1}{1-a}}+\int_{U}\left|\nabla \Psi_{t}(x, t)\right|^{2}+\left|\Psi_{t t}(x, t)\right|^{2} d x, t \geq 0 .
$$

We have $\tilde{A}(\gamma, t) \leq S(\gamma, t)$. Regarding $G_{1}(t)$ as part of $G_{4}(t)$, we note that if $\gamma \geq$ $\max \left\{2-a, r_{0}\right\}$ then

$$
\begin{aligned}
{\left[\int_{U}\left|\Psi_{t}\right|^{r_{0}} d x\right]^{\frac{1}{r_{0}}} } & \leq 1+\left[\int_{U}\left|\Psi_{t}\right|^{r_{0}} d x\right]^{\frac{2-a}{r_{0}(1-a)}} \leq 1+C\left[\int_{U}\left|\Psi_{t}\right|^{\gamma} d x\right]^{\frac{2-a}{\gamma(1-a)}} \\
& \leq C+C\left[\int_{U}\left|\Psi_{t}\right|^{\gamma} d x\right]^{\frac{1}{1-a}}
\end{aligned}
$$

hence we easily find $G_{4}(t) \leq C(1+S(\gamma, t))$. Therefore, if $\alpha \geq 2, \lambda>0$ and $t>0$ then

$$
R_{1}(\alpha, t)+\tilde{A}(\lambda, t)+G_{4}(t) \leq C(1+S(\gamma, t)), \text { where } \gamma=\max \left\{\alpha, 4-2 a, \lambda, r_{0}\right\}
$$

By (6.39) and (6.40), we obtain

$$
\bar{R}(t) \leq C \overline{\bar{R}}(t), \quad t>0
$$


where

$$
\overline{\bar{R}}(t)=\overline{\bar{R}}(\alpha, t) \stackrel{\text { def }}{=} M_{0}+\operatorname{Env} S\left(\tilde{\gamma}_{5}, t\right)
$$

with $\tilde{\gamma}_{5}=\max \left\{\alpha, 4-2 a, \gamma_{5}, r_{0}\right\}$. Since $\gamma_{5} \geq \gamma_{2} \geq 2 \alpha \geq 6>4-2 a$, we have

$$
\tilde{\gamma}_{5}=\max \left\{\gamma_{5}, r_{0}\right\}
$$

Thanks to (6.36) and (6.41),

$$
R(t) \leq C \overline{\bar{R}}(t), \quad t \geq 1
$$

Theorem 6.3. Assume (DC) and $\alpha \geq 3$.

(i) If $t \geq 1$ then

$$
\begin{aligned}
\int_{U}|z(x, t)|^{\alpha} d x \leq & \int_{U}|z(x, 0)|^{\alpha} d x \\
& +C\left|\vec{a}^{(1)}-\vec{a}^{(2)}\right|\left\{M_{0,1}+\int_{1}^{t} e^{-c_{11} \int_{\tau}^{t} \bar{M}_{1}(s)^{-\frac{a}{2-a}}} d s \overline{\bar{R}}(\tau) d \tau\right\},
\end{aligned}
$$

where $c_{11}=c_{11}(\alpha)>0$, and $M_{0,1}$ is the constant $M_{0, T}$ defined by (6.24) with $T=1$.

(ii) Let $\gamma_{6}=\max \left\{\gamma_{4}, \gamma_{5}, r_{0}\right\}$. If

$$
\Upsilon_{0} \stackrel{\text { def }}{=} 1+\sum_{k=1}^{2} \int_{U}\left|\bar{p}_{k}(x, 0)\right|^{\gamma_{6}}+\left|\nabla p_{k}(x, 0)\right|^{2-a} d x+\sup _{[0, \infty)} S\left(\gamma_{6}, t\right)
$$

is finite then

$$
\sup _{[0, \infty)} \int_{U}|z(x, t)|^{\alpha} d x \leq \int_{U}|z(x, 0)|^{\alpha} d x+C \Upsilon_{0}^{\frac{2}{2-a}}\left|\vec{a}^{(1)}-\vec{a}^{(2)}\right|
$$

Proof. (i) By virtue of (6.32) and (6.44), we can replace $M_{1}(t)$ and $R(t)$ by $\bar{M}_{1}(t)$ and $\overline{\bar{R}}(t)$, respectively, in (6.3) for $t \geq 1$, and then apply Gronwall's inequality to obtain

$$
\int_{U}|z(x, t)|^{\alpha} d x \leq \int_{U}|z(x, 1)|^{\alpha} d x+C\left|\vec{a}^{(1)}-\vec{a}^{(2)}\right| \int_{1}^{t} e^{-c_{11} \int_{\tau}^{t} \bar{M}_{1}(s)^{-\frac{a}{2-a}}} d s \overline{\bar{R}}(\tau) d \tau .
$$

Theorem 6.2 with $T=1$ yields

$$
\sup _{[0,1]} \int_{U}|z(x, t)|^{\alpha} d x \leq \int_{U}|z(x, 0)|^{\alpha} d x+C M_{0,1}\left|\vec{a}^{(1)}-\vec{a}^{(2)}\right| .
$$

Then using (6.48) to estimate $\int_{U}|z(x, 1)|^{\alpha} d x$ in (6.47) results in (6.45).

(ii) Note that $M_{0,1}, M_{0}, \bar{M}_{1}(t) \leq C \Upsilon_{0}$. Also, $\tilde{\gamma}_{5} \leq \gamma_{6}$ implies

$$
S\left(\tilde{\gamma}_{5}, t\right) \leq C\left(1+S\left(\gamma_{6}, t\right)\right) \leq C \Upsilon_{0},
$$

hence we can select $\operatorname{Env} S\left(\tilde{\gamma}_{5}, t\right) \equiv C \Upsilon_{0}$. Therefore it follows from (6.45) for $t \geq 1$ that

$$
\int_{U}|z(x, t)|^{\alpha} d x \leq \int_{U}|z(x, 0)|^{\alpha} d x+C\left|\vec{a}^{(1)}-\vec{a}^{(2)}\right|\left[\Upsilon_{0}+\int_{1}^{t} e^{-C \Upsilon_{0}^{-\frac{a}{2-a}}(t-\tau)} \Upsilon_{0} d \tau\right],
$$

thus

$$
\int_{U}|z(x, t)|^{\alpha} d x \leq \int_{U}|z(x, 0)|^{\alpha} d x+C \Upsilon_{0}^{\frac{2}{2-a}}\left|\vec{a}^{(1)}-\vec{a}^{(2)}\right|
$$


Combining estimate (6.49) for $t \geq 1$ with (6.48), we obtain (6.46).

Theorem 6.4. Assume (NDC) and $\alpha \geq \max \left\{3, \alpha_{*}\right\}$.

(i) If $t \geq 1$ then

$$
\begin{aligned}
\int_{U}|z(x, t)|^{\alpha} d x \leq & \int_{U}|z(x, 0)|^{\alpha} d x+C M_{0,1}\left|\vec{a}^{(1)}-\vec{a}^{(2)}\right| \\
& +C\left|\vec{a}^{(1)}-\vec{a}^{(2)}\right|^{\frac{1}{\theta}}\left[\operatorname{Env}\left(\bar{R}(t) \bar{M}_{2}(t)^{\frac{2-\theta_{1}}{\theta_{1}}}\right)\right]^{\frac{1}{\theta}} .
\end{aligned}
$$

(ii) Let $\gamma_{7}=\gamma_{7}(\alpha)=\max \left\{\theta_{2} \alpha, \gamma_{4}, \gamma_{5}, r_{0}\right\}$. If

$$
\Upsilon_{0} \stackrel{\text { def }}{=} 1+\sum_{k=1}^{2} \int_{U}\left|\bar{p}_{k}(x, 0)\right|^{\gamma_{7}}+\left|\nabla p_{k}(x, 0)\right|^{2-a} d x+\sup _{[0, \infty)} S\left(\gamma_{7}, t\right)
$$

is finite then

$$
\sup _{[0, \infty)} \int_{U}|z(x, t)|^{\alpha} d x \leq \int_{U}|z(x, 0)|^{\alpha} d x+C \Upsilon_{0}\left|\vec{a}^{(1)}-\vec{a}^{(2)}\right|+C \Upsilon_{0}^{\frac{2}{\theta_{1} \theta}}\left|\vec{a}^{(1)}-\vec{a}^{(2)}\right|^{\frac{1}{\theta}}
$$

Proof. (i) By virtue of (6.32) and (6.36), we replace $M_{2}(t), R(t)$ by $\bar{M}_{2}(t), \bar{R}(t)$, respectively, in inequality (6.6) for $t \geq 1$ to obtain

$$
\frac{d}{d t} \int_{U}|z|^{\alpha} d x \leq-C_{1}\left[\int_{U}|z|^{\alpha} d x\right]^{\theta} \bar{M}_{2}(t)^{-\frac{2-\theta_{1}}{\theta_{1}}}+C_{2}\left|\vec{a}^{(1)}-\vec{a}^{(2)}\right| \bar{R}(t) .
$$

Applying Lemma 2.7 to the preceding inequality on $[1, \infty)$ yields

$$
\int_{U}|z(x, t)|^{\alpha} d x \leq \int_{U}|z(x, 1)|^{\alpha} d x+C\left|\vec{a}^{(1)}-\vec{a}^{(2)}\right|^{\frac{1}{\theta}}\left[\operatorname{Env}\left(\bar{R}(t) \bar{M}_{2}(t)^{\frac{2-\theta_{1}}{\theta_{1}}}\right)\right]^{\frac{1}{\theta}} .
$$

Again, using (6.48) to estimate $\int_{U}|z(x, 1)|^{\alpha} d x$ in (6.52) yields (6.50).

(ii) We have $M_{0,1}, M_{0} \leq C \Upsilon_{0}$, and $\bar{M}_{2}(t) \leq C \Upsilon_{0}$ for all $t \geq 0$. Also, thanks to (6.41), $\bar{R}(t) \leq C \overline{\bar{R}}(t) \leq C \Upsilon_{0}$ for all $t \geq 1$. Employing these in (6.50), we have for $t \geq 1$ that

$$
\begin{aligned}
\int_{U}|z(x, t)|^{\alpha} d x & \leq \int_{U}|z(x, 0)|^{\alpha} d x+C \Upsilon_{0}\left|\vec{a}^{(1)}-\vec{a}^{(2)}\right|+C\left|\vec{a}^{(1)}-\vec{a}^{(2)}\right| \frac{1}{\theta}\left(\Upsilon_{0} \Upsilon_{0}^{\frac{2-\theta_{1}}{\theta_{1}}}\right)^{\frac{1}{\theta}} \\
& =\int_{U}|z(x, 0)|^{\alpha} d x+C \Upsilon_{0}\left|\vec{a}^{(1)}-\vec{a}^{(2)}\right|+C \Upsilon_{0}^{\frac{2}{\theta \theta_{1}}}\left|\vec{a}^{(1)}-\vec{a}^{(2)}\right|^{\frac{1}{\theta}} .
\end{aligned}
$$

Combining this with (6.48), we obtain (6.51).

We now study the limit superior of $\int_{U}|z(x, t)|^{\alpha} d x$ as $t \rightarrow \infty$.

In the DC case, if $\int_{0}^{\infty} M_{1}(t)^{-a /(2-a)} d t=\infty$ then by (6.3) and Lemma 2.7 we have

$$
\limsup _{t \rightarrow \infty} \int_{U}|z(x, t)|^{\alpha} d x \leq C\left|\vec{a}^{(1)}-\vec{a}^{(2)}\right| \limsup _{t \rightarrow \infty}\left(R(t) \cdot M_{1}(t)^{\frac{a}{2-a}}\right) .
$$

Similarly, in the NDC case, if $\int_{0}^{\infty} M_{2}(t)^{-\frac{2-\theta_{1}}{\theta_{1}}} d t=\infty$ then by (6.6) and Lemma 2.7 we have

$$
\limsup _{t \rightarrow \infty} \int_{U}|z(x, t)|^{\alpha} d x \leq C\left|\vec{a}^{(1)}-\vec{a}^{(2)}\right|^{\frac{1}{\theta}} \limsup _{t \rightarrow \infty}\left(R(t) \cdot M_{2}(t)^{\frac{\theta_{1}}{2-\theta_{1}}}\right)^{\frac{1}{\theta}} .
$$

Hence we need to estimate the limits superior of $M_{1}(t), M_{2}(t)$ and $R(t)$. 
Let $\alpha \geq \max \left\{3, \alpha_{*}\right\}$. In (6.35), by applying Theorem 4.3(ii), with $\alpha$ set to be $\gamma_{5}$, and Theorem 4.5(ii) combined with relation (6.30) we have

$$
\limsup _{t \rightarrow \infty} R(t) \leq C \limsup _{t \rightarrow \infty}\left(1+R_{1}(t)+A\left(\gamma_{3}+2, t\right)+\tilde{A}\left(\widehat{\gamma_{5}}, t\right)+\tilde{A}(\widehat{2}, t)+G_{4}(t)\right)
$$

Since $\gamma_{5} \geq \max \left\{\widehat{2}, 2 \alpha, \gamma_{3}+2\right\}$, then $\widehat{\gamma_{5}}=\gamma_{5}$ and by taking into account (6.22), (6.30), (6.31) and (6.40) we assert

$$
\limsup _{t \rightarrow \infty} R(t) \leq C \limsup _{t \rightarrow \infty}\left(1+R_{1}(t)+\tilde{A}\left(\gamma_{5}, t\right)+G_{4}(t)\right) \leq C \limsup _{t \rightarrow \infty}\left(1+S\left(\tilde{\gamma}_{5}, t\right)\right) .
$$

Similarly, from part (ii) of Theorems 4.4 and 4.3 follow

$$
\limsup _{t \rightarrow \infty} M_{1}(t) \leq C \limsup _{t \rightarrow \infty}\left(1+\tilde{A}(\widehat{2}, t)+G_{3}(t)\right) \leq C \limsup _{t \rightarrow \infty}\left(1+S\left(\tilde{\gamma}_{5}, t\right)\right),
$$

$\limsup _{t \rightarrow \infty} M_{2}(t) \leq C \limsup _{t \rightarrow \infty}\left(1+\tilde{A}(\widehat{2}, t)+G_{3}(t)+\tilde{A}\left(\widehat{\theta_{2} \alpha}, t\right)\right) \leq C \limsup _{t \rightarrow \infty}\left(1+S\left(\gamma_{8}, t\right)\right)$,

where $\gamma_{8}=\gamma_{8}(\alpha)=\max \left\{\gamma_{5}, \theta_{2} \alpha, r_{0}\right\}$

Theorem 6.5. (i) In the DC case, if $\alpha \geq 3$ and $\Upsilon_{1} \stackrel{\text { def }}{=} \lim \sup _{t \rightarrow \infty} S\left(\gamma_{5}, t\right)$ is finite, then

$$
\limsup _{t \rightarrow \infty} \int_{U}|z(x, t)|^{\alpha} d x \leq C\left(1+\Upsilon_{1}\right)^{\frac{2}{2-a}}\left|\vec{a}^{(1)}-\vec{a}^{(2)}\right| \text {. }
$$

(ii) In the NDC case, if $\alpha \geq \max \left\{3, \alpha_{*}\right\}$ and

$$
\Upsilon_{2}=\Upsilon_{2}(\alpha) \stackrel{\text { def }}{=} \limsup _{t \rightarrow \infty} S\left(\gamma_{8}, t\right)
$$

is finite, then

$$
\limsup _{t \rightarrow \infty} \int_{U}|z(x, t)|^{\alpha} d x \leq C\left(1+\Upsilon_{2}\right)^{\frac{2}{\theta \theta_{1}}}\left|\vec{a}^{(1)}-\vec{a}^{(2)}\right|^{\frac{1}{\theta}}
$$

Proof. (i) Assume (DC), $\alpha \geq 3$ and $\Upsilon_{1}<\infty$. By (2.17) We have $\widehat{2}=2, \widehat{3}=3$ and $r_{0} \leq 2$. Hence $\alpha \geq \max \left\{3, \alpha_{*}\right\}$ and $\tilde{\gamma}_{5}=\gamma_{5}$. We have $1+S\left(\gamma_{5}, t\right) \leq C$ for all $t \geq 0$. Thanks to this uniform boundedness, we have from (6.53), (6.55) and (6.56) that $\limsup _{t \rightarrow \infty} \int_{U}|z(x, t)|^{\alpha} d x \leq C\left|\vec{a}^{(1)}-\vec{a}^{(2)}\right| \limsup _{t \rightarrow \infty}\left(1+S\left(\gamma_{5}, t\right)\right) \limsup _{t \rightarrow \infty}\left(1+S\left(\gamma_{5}, t\right)\right)^{\frac{a}{2-a}}$ which proves (6.58).

(ii) Assume (NDC), $\alpha \geq \max \left\{3, \alpha_{*}\right\}$ and $\Upsilon_{2}<\infty$. Note that $\gamma_{5} \geq \gamma_{2} \geq 2 \alpha>\alpha_{*}$ and $r_{0}>2$. Similar to part (i), we obtain from (6.54), (6.55) and (6.57) that

$$
\begin{aligned}
& \limsup _{t \rightarrow \infty} \int_{U}|z(x, t)|^{\alpha} d x \\
& \quad \leq C\left|\vec{a}^{(1)}-\vec{a}^{(2)}\right|^{\frac{1}{\theta}} \limsup _{t \rightarrow \infty}\left(1+S\left(\tilde{\gamma}_{5}, t\right)\right)^{\frac{1}{\theta}} \limsup _{t \rightarrow \infty}\left(1+S\left(\gamma_{8}, t\right)\right)^{\frac{2-\theta_{1}}{\theta \theta_{1}}} .
\end{aligned}
$$


Since $\tilde{\gamma}_{5} \leq \gamma_{8}$, we have $S\left(\tilde{\gamma}_{5}, t\right) \leq C\left(1+S\left(\gamma_{8}, t\right)\right)$. Thus (6.61) gives

$$
\limsup _{t \rightarrow \infty} \int_{U}|z(x, t)|^{\alpha} d x \leq C\left|\vec{a}^{(1)}-\vec{a}^{(2)}\right|^{\frac{1}{\theta}}\left(1+\limsup _{t \rightarrow \infty} S\left(\gamma_{8}, t\right)\right)^{\frac{1}{\theta}+\frac{2-\theta_{1}}{\theta \theta_{1}}}
$$

and (6.59) follows.

We now find estimates for $\int_{U}|\nabla z(x, t)|^{2-a} d x$. Since the DC case was already studied in [14], we focus on the NDC case here. Recall that $\widehat{3}=\max \left\{3, \alpha_{*}\right\}$.

Theorem 6.6. Assume (NDC).

(i) For all $t \geq 1$,

$$
\begin{aligned}
& \left(\int_{U}|\nabla z(x, t)|^{2-a} d x\right)^{\frac{2}{2-a}} \leq C\left|\vec{a}^{(1)}-\vec{a}^{(2)}\right| \bar{M}_{1}(t)^{\frac{2}{2-a}} \\
& +C \bar{M}_{1}(t)^{\frac{a}{2-a}} \bar{M}_{3}(t)^{\frac{1}{2}}\left\{\left[\int_{U}|z(x, 0)|^{\widehat{3}} d x\right]^{1 / \widehat{3}}\right. \\
& \left.+\left[M_{0,1}\left|\vec{a}^{(1)}-\vec{a}^{(2)}\right|+\left(\operatorname{Env}\left\{\bar{R}(\widehat{3}, t) \bar{M}_{2, \widehat{3}}(t)^{\frac{2-\theta_{1}}{\theta_{1}}}\right\}\right)^{\frac{1}{\theta}}\left|\vec{a}^{(1)}-\vec{a}^{(2)}\right|^{\frac{1}{\theta}}\right]^{1 / \widehat{3}}\right\},
\end{aligned}
$$

where $\bar{M}_{3}(t)$ is defined in (5.61) and (5.60) with the boundary data $\Psi_{1}=\Psi_{2}=\Psi$ and the difference $\Phi=0$, while $M_{0,1}$ is defined by (6.24) and $\bar{M}_{2, \widehat{3}}(t)$ is defined by (6.34) with $\alpha=\widehat{3}$.

(ii) Let $\Upsilon_{2}=\Upsilon_{2}(\widehat{3})$, the limit defined by (6.59) with $\alpha=\widehat{3}$. If $\Upsilon_{2}<\infty$ then

$$
\begin{aligned}
\limsup _{t \rightarrow \infty}\left(\int_{U}|\nabla z(x, t)|^{2-a} d x\right)^{\frac{2}{2-a}} \leq C\left(1+\Upsilon_{2}\right)^{\frac{2}{2-a}}\left|\vec{a}^{(1)}-\vec{a}^{(2)}\right| \\
+C\left(1+\Upsilon_{2}\right)^{1 / 2+a /(2-a)+2 /\left(\widehat{3} \theta \theta_{1}\right)}\left|\vec{a}^{(1)}-\vec{a}^{(2)}\right|^{1 /(\widehat{3} \theta)} .
\end{aligned}
$$

Proof. (i) Inequality (5.20) of Lemma 5.3 in [13] reads

$$
\begin{aligned}
\int_{U} z z_{t} d x \leq & -C\left(\int_{U}|\nabla z|^{2-a} d x\right)^{\frac{2}{2-a}} M_{1}(t)^{\frac{-a}{2-a}} \\
& +C\left|\vec{a}^{(1)}-\vec{a}^{(2)}\right| \int_{U}\left(\left|\nabla p_{1}\right|^{2-a}+\left|\nabla p_{2}\right|\right)^{2-a} d x
\end{aligned}
$$

hence

$$
\left(\int_{U}|\nabla z|^{2-a} d x\right)^{\frac{2}{2-a}} \leq C\left|\vec{a}^{(1)}-\vec{a}^{(2)}\right| M_{1}(t)^{\frac{2}{2-a}}+C M_{1}(t)^{\frac{a}{2-a}} \int_{U}|z|\left|z_{t}\right| d x .
$$

Same note as above, the constant $C$ here can be made dependent on $\hat{\chi}(\mathbf{D})$ instead of on $\vec{a}^{(1)}$ and $\vec{a}^{(2)}$. Since $z_{t}=\bar{z}_{t}$ and $\widehat{3}>2$, applying Hölder's inequality to the last integral we have

$\left[\int_{U}|\nabla z|^{2-a} d x\right]^{\frac{2}{2-a}} \leq C\left|\vec{a}^{(1)}-\vec{a}^{(2)}\right| M_{1}(t)^{\frac{2}{2-a}}+C M_{1}(t)^{\frac{a}{2-a}}\left[\int_{U}\left|\bar{z}_{t}\right|^{2} d x\right]^{\frac{1}{2}}\left[\int_{U}|z|^{\widehat{3}} d x\right]^{1 / \widehat{3}}$.

We use (6.32) to bound $M_{1}(t)$, use (5.61) to bound

$$
\int_{U}\left|\bar{z}_{t}\right|^{2} d x \leq 2 \int_{U}\left|\left(\bar{p}_{1}\right)_{t}\right|^{2}+\left|\left(\bar{p}_{2}\right)_{t}\right|^{2} d x \leq C \bar{M}_{3}(t)
$$


and use (6.50) with $\alpha=\widehat{3}$ to estimate $\int_{U}|z|^{\widehat{3}} d x$, hence obtain

$$
\begin{aligned}
& \left(\int_{U}|\nabla z|^{2-a} d x\right)^{\frac{2}{2-a}} \leq C\left|\vec{a}^{(1)}-\vec{a}^{(2)}\right| \bar{M}_{1}(t)^{\frac{2}{2-a}}+C \bar{M}_{1}(t)^{\frac{a}{2-a}} \bar{M}_{3}(t)^{\frac{1}{2}}\left\{\int_{U}|z(x, 0)|^{\widehat{3}} d x\right. \\
& \left.+M_{0,1}\left|\vec{a}^{(1)}-\vec{a}^{(2)}\right|+\left[\operatorname{Env}\left(\bar{R}(\widehat{3}, t) \bar{M}_{2, \widehat{3}}(t)^{\frac{2-\theta_{1}}{\theta_{1}}}\right)\right]^{\frac{1}{\theta}}\left|\vec{a}^{(1)}-\vec{a}^{(2)}\right|^{\frac{1}{\theta}}\right\}^{1 / \widehat{3}} .
\end{aligned}
$$

This proves inequality (6.62).

(ii) Assume $\Upsilon_{2}<\infty$. Let $R(t)=R(\widehat{3}, t)$ and $S(t)=S\left(\gamma_{8}(\widehat{3}), t\right)$ defined as in Lemma 6.1 and Theorem 6.5 , respectively, with $\alpha=\widehat{3}$. Similar to the previous theorem, one can easily verify that

$$
\limsup _{t \rightarrow \infty} M_{1}(t), \limsup _{t \rightarrow \infty} R(t) \leq C\left(1+\limsup _{t \rightarrow \infty} S(t)\right) \leq C\left(1+\Upsilon_{2}\right)
$$

Note from the definition of $R(t)$ in Lemma 6.1 that

$$
\int_{U}\left|\bar{z}_{t}\right|^{2} d x \leq 2 \int_{U}\left|\left(\bar{p}_{1}\right)_{t}\right|^{2}+\left|\left(\bar{p}_{2}\right)_{t}\right|^{2} d x \leq 2 R_{2}(t) \leq 2 R(t) .
$$

We then have

$$
\limsup _{t \rightarrow \infty} \int_{U}\left|\bar{z}_{t}(x, t)\right|^{2} d x \leq 2 \limsup _{t \rightarrow \infty} R(t) \leq C\left(1+\Upsilon_{2}\right)
$$

By (6.65), (6.66) and (6.60) with $\alpha=\widehat{3}$, each time-dependent term on the right-hand side of (6.64) has finite limit superior. Hence taking the limit superior of inequality (6.64) and using the mentioned estimates we obtain

$$
\begin{aligned}
& \limsup _{t \rightarrow \infty}\left(\int_{U}|\nabla z(x, t)|^{2-a} d x\right)^{\frac{2}{2-a}} \leq C\left|\vec{a}^{(1)}-\vec{a}^{(2)}\right|\left(1+\Upsilon_{2}\right)^{\frac{2}{2-a}} \\
& \quad+C\left(1+\Upsilon_{2}\right)^{\frac{a}{2-a}}\left(1+\Upsilon_{2}\right)^{\frac{1}{2}}\left\{\left(1+\Upsilon_{2}\right)^{\frac{2}{\theta \theta}}\left|\vec{a}^{(1)}-\vec{a}^{(2)}\right|^{\frac{1}{\theta}}\right\}^{1 / \widehat{3}}
\end{aligned}
$$

therefore (6.63) follows.

\section{APPENDIX A}

We generalize the results in section 3 of [14]. This covers the special case in Lemma 2.7.

Lemma A.1. Let $\phi$ be a continuous, strictly increasing function from $[0, \infty)$ onto $[0, \infty)$. Suppose $y(t) \geq 0$ is a continuous function on $[0, \infty)$ such that

$$
y^{\prime} \leq-h(t) \phi^{-1}(y(t))+f(t), \quad t>0,
$$

where $h(t)>0, f(t) \geq 0$ for $t \geq 0$ are continuous functions on $[0, \infty)$.

(i) If $M(t)=\operatorname{Env}\left(\frac{f(t)}{h(t)}\right)$ on $[0, \infty)$ then

$$
y(t) \leq y(0)+\phi(M(t)) \text { for all } t \geq 0 .
$$

(ii) If $\int_{0}^{\infty} h(\tau) d \tau=\infty$ then

$$
\limsup _{t \rightarrow \infty} y(t) \leq \phi\left(\limsup _{t \rightarrow \infty} \frac{f(t)}{h(t)}\right)
$$


Here, we use the notation $\phi(\infty)=\infty$.

Proof. (i) Claim 1. Given any $\varepsilon>0$,

$$
y(t) \leq y(0)+\varepsilon+\phi(M(t)) \text { for all } t \geq 0 .
$$

Suppose (A.4) fails. Then there exist $t_{2}>t_{1}>0$ such that

$$
\begin{aligned}
& y\left(t_{1}\right)=y(0)+\phi\left(M\left(t_{1}\right)\right), \\
& y\left(t_{2}\right)=y(0)+\varepsilon+\phi\left(M\left(t_{2}\right)\right), \\
& y(t) \geq y(0)+\phi(M(t)) \geq \phi\left(\frac{f(t)}{h(t)}\right), \text { for all } t \in\left[t_{1}, t_{2}\right) .
\end{aligned}
$$

The last inequality and (A.1) give

$$
y^{\prime}(t) \leq-h(t) \phi^{-1}(y(t))+f(t) \leq-h(t) \frac{f(t)}{h(t)}+f(t)=0
$$

for all $t \in\left(t_{1}, t_{2}\right)$, thus $y(t)$ is decreasing on $\left[t_{1}, t_{2}\right]$. This and the fact that $M(t)$ is increasing give

$$
y\left(t_{2}\right) \leq y\left(t_{1}\right)=y(0)+\phi\left(M\left(t_{1}\right)\right)<y(0)+\varepsilon+\phi\left(M\left(t_{2}\right)\right)=y\left(t_{2}\right) .
$$

It is a contradiction and hence Claim 1 holds true. Now letting $\varepsilon \rightarrow 0$ in (A.4) yields (A.2).

(ii) Assume $\int_{0}^{\infty} h(\tau) d \tau=\infty$. Let $A=\lim \sup _{t \rightarrow \infty} \phi\left(\frac{f(t)}{h(t)}\right)$. It suffices to prove (A.3) for finite $A$. Given $\delta>0$, there is $T_{0}>0$ such that for all $t>T_{0}$

$$
\phi\left(\frac{f(t)}{h(t)}\right) \leq A+\delta \Rightarrow f(t) \leq \phi^{-1}(A+\delta) h(t) .
$$

Thus

$$
y^{\prime}(t) \leq-h(t) \phi^{-1}(y(t))+\phi^{-1}(A+\delta) h(t) \text { for all } t>T_{0} .
$$

Claim 2. Given any $\varepsilon>0$, there is $T>0$ such that

$$
y(t) \leq \phi\left(\phi^{-1}(A+\delta)+\varepsilon\right) \text { for all } t>T .
$$

We infer from (A.6) that

$$
\limsup _{t \rightarrow \infty} y(t) \leq \phi\left(\phi^{-1}(A+\delta)+\varepsilon\right) .
$$

Now letting $\varepsilon \rightarrow 0$ and then $\delta \rightarrow 0$, and using the continuity of $\phi$ and $\phi^{-1}$ we obtain (A.3).

Proof of Claim 2. Given $\varepsilon>0$. If (A.6) holds for $T=T_{0}$ then we are done. Suppose (A.6) fails for $T=T_{0}$, we will prove that (A.6) holds for some $T>T_{0}$. Because (A.6) fails for $T=T_{0}$, then there is $T_{*} \geq T_{0}$ such that

$$
y\left(T_{*}\right)>\phi\left(\phi^{-1}(A+\delta)+\varepsilon\right) .
$$


We claim that there is $t_{*}>T_{*}$ such that

$$
y\left(t_{*}\right) \leq \phi\left(\phi^{-1}(A+\delta)+\frac{\varepsilon}{2}\right) .
$$

Indeed, if this is not the case then

$$
y(t)>\phi\left(\phi^{-1}(A+\delta)+\frac{\varepsilon}{2}\right) \text { for all } t>T_{*} .
$$

It implies for all $t>T_{*}$ that

$$
y^{\prime}(t) \leq-h(t)\left[\phi^{-1}(A+\delta)+\frac{\varepsilon}{2}\right]+\phi^{-1}(A+\delta) h(t)=-\frac{\varepsilon}{2} h(t) .
$$

Thus for $t>T_{*}$,

$$
y(t)=y\left(T_{*}\right)+\int_{T_{*}}^{t} y^{\prime}(\tau) d \tau \leq y\left(T_{*}\right)-\frac{\varepsilon}{2} \int_{T_{*}}^{t} h(\tau) d \tau \rightarrow-\infty \text { as } t \rightarrow \infty,
$$

since $\int_{0}^{\infty} h(\tau) d \tau=\infty$ and $h(t)$ is continuous on $\left[0, T_{*}\right]$. This contradicts the fact that $y(t) \geq 0$ for all $t \geq 0$ and hence (A.8) must be true.

By (A.7) and (A.8) there exists $T \in\left(T_{*}, t_{*}\right]$ such that

$$
\phi\left(\phi^{-1}(A+\delta)+\frac{\varepsilon}{2}\right)=y(T)<\phi\left(\phi^{-1}(A+\delta)+\varepsilon\right) .
$$

Now we prove that (A.6) holds for such $T$. Suppose otherwise, then there is $T^{\prime}>T$ such that $y\left(T^{\prime}\right)>\phi\left(\phi^{-1}(A+\delta)+\varepsilon\right)$. Hence there are $t_{1}$ and $t_{2}$ with $T \leq t_{1}<t_{2} \leq T^{\prime}$ such that

$$
\phi\left(\phi^{-1}(A+\delta)+\frac{\varepsilon}{2}\right) \leq y(t)<y\left(t_{2}\right)=\phi\left(\phi^{-1}(A+\delta)+\varepsilon\right), \text { for all } t_{1} \leq t<t_{2} .
$$

Then we have from (A.5) and (A.9) that

$$
\begin{aligned}
y\left(t_{2}\right)-y\left(t_{1}\right) & =\int_{t_{1}}^{t_{2}} y^{\prime}(t) d t \leq \int_{t_{1}}^{t_{2}}-h(t) \phi^{-1}(y(t))+\phi^{-1}(A+\delta) h(t) d t \\
& \leq \int_{t_{1}}^{t_{2}}-h(t)\left(\phi^{-1}(A+\delta)+\frac{\varepsilon}{2}\right)+\phi^{-1}(A+\delta) h(t) d t \\
& =\int_{t_{1}}^{t_{2}}-\frac{\varepsilon}{2} h(t) d t<0,
\end{aligned}
$$

since $h(t)>0$ and $h(t)$ is continuous on $\left[t_{1}, t_{2}\right]$. Thus $y\left(t_{2}\right)<y\left(t_{1}\right)$ which contradicts (A.9) with $t=t_{1}$. Therefore (A.6) holds for $T$. The proof is complete.

Acknowledgments. We would like to thank Eugenio Aulisa for helpful discussions. L.H. and A.I. acknowledge the support by NSF Grant DMS-0908177.

\section{REFERENCES}

[1] Antontsev, S. N., AND Diaz, J. I. On space or time localization of solutions of nonlinear elliptic or parabolic equations via energy methods. In Recent advances in nonlinear elliptic and parabolic problems (Nancy, 1988), vol. 208 of Pitman Res. Notes Math. Ser. Longman Sci. Tech., Harlow, 1989, pp. 3-14.

[2] Aulisa, E., Bloshanskaya, L., Hoang, L., And Ibragimov, A. Analysis of generalized Forchheimer flows of compressible fluids in porous media. J. Math. Phys. 50, 10 (2009), 103102, 44. 
[3] Balhoff, M., Mikelić, A., And Wheeler, M. F. Polynomial filtration laws for low Reynolds number flows through porous media. Transp. Porous Media 81, 1 (2010), 35-60.

[4] BeAR, J. Dynamics of Fluids in Porous Media. Dover, New York, 1972.

[5] BRÉZIS, H. Opérateurs maximaux monotones et semi-groupes de contractions dans les espaces de Hilbert. North-Holland Publishing Co., Amsterdam, 1973. North-Holland Mathematics Studies, No. 5. Notas de Matemática (50).

[6] BRÉZIS, H., AND LIEB, E. A relation between pointwise convergence of functions and convergence of functionals. Proc. Amer. Math. Soc. 88, 3 (1983), 486-490.

[7] Çelebi, A. O., Kalantarov, V. K., AND UĞURlu, D. On continuous dependence on coefficients of the Brinkman-Forchheimer equations. Appl. Math. Lett. 19, 8 (2006), 801-807.

[8] Çelebi, A. O., Kalantarov, V. K., And UĞURlu, D. Structural stability for the double diffusive convective Brinkman equations. Appl. Anal. 87, 8 (2008), 933-942.

[9] Chadam, J., AND QIn, Y. Spatial decay estimates for flow in a porous medium. SIAM J. Math. Anal. 28, 4 (1997), 808-830.

[10] Darcy, H. Les Fontaines Publiques de la Ville de Dijon. Dalmont, Paris, 1856.

[11] DiBenedetto, E. Degenerate parabolic equations. Universitext. Springer-Verlag, New York, 1993.

[12] DiBenedetto, E., Gianazza, U., And Vespri, V. Forward, backward and elliptic Harnack inequalities for non-negative solutions to certain singular parabolic partial differential equations. Ann. Sc. Norm. Super. Pisa Cl. Sci. (5) 9, 2 (2010), 385-422.

[13] HoAng, L., AND IbRAGimov, A. Structural stability of generalized Forchheimer equations for compressible fluids in porous media. Nonlinearity 24, 1 (2011), 1-41.

[14] Hoang, L., And Ibragimov, A. Qualitative study of generalized forchheimer flows with the flux boundary condition. Adv. Diff. Eq. 17, 5-6 (2012), 511-556.

[15] Lin, C., AND Payne, L. E. Structural stability for a Brinkman fluid. Math. Methods Appl. Sci. 30, 5 (2007), 567-578.

[16] Lions, J.-L. Quelques méthodes de résolution des problèmes aux limites non linéaires. Dunod, 1969.

[17] Muskat, M. The flow of homogeneous fluids through porous media. McGraw-Hill Book Company, inc., 1937.

[18] Payne, L. E., And Song, J. C. Spatial decay bounds for double diffusive convection in Brinkman flow. J. Differential Equations 244, 2 (2008), 413-430.

[19] Payne, L. E., Song, J. C., And Straughan, B. Continuous dependence and convergence results for Brinkman and Forchheimer models with variable viscosity. R. Soc. Lond. Proc. Ser. A Math. Phys. Eng. Sci. 455, 1986 (1999), 2173-2190.

[20] SHOwALTER, R. E. Monotone operators in Banach space and nonlinear partial differential equations, vol. 49 of Mathematical Surveys and Monographs. American Mathematical Society, Providence, RI, 1997.

[21] Straughan, B. Stability and wave motion in porous media, vol. 165 of Applied Mathematical Sciences. Springer, New York, 2008.

[22] Straughan, B. Structure of the dependence of Darcy and Forchheimer coefficients on porosity. Internat. J. Engrg. Sci. 48, 11 (2010), 1610-1621.

[23] ZeIDLER, E. Nonlinear functional analysis and its applications. II/B. Springer-Verlag, New York, 1990. Nonlinear monotone operators, Translated from the German by the author and Leo F. Boron.

${ }^{\dagger}$ Department of Mathematics and Statistics, Texas Tech University, Box 41042 LubBOCK, TX 79409-1042, U.S.A.

E-mail address: luan.hoang@ttu.edu

E-mail address: akif.ibraguimov@ttu.edu

E-mail address: thinh.kieu@ttu.edu

‡Department of Mathematics, Swansea University, Singleton Park, Swansea, Sa2 8PP, WALES, U.K.

E-mail address: Z. Sobol@swansea.ac.uk

* Corresponding AUthor 